WSRC-TR-97-00289, Rev. 1

\title{
A Summary Report on the NPH Evaluation of 105-L Disassembly Basin
}

by

J. R. Joshi

Westinghouse Savannah River Company

Savannah River Site

Aiken, South Carolina 29808

J. A. Amin

This paper was prepared in connection with work done under the above contract number with the U. S. Department of Energy. By acceptance of this paper, the publisher and/or recipient acknowledges the U.S. Government's right to retain a nonexclusive, royalty-free license in and to any copyright covering this paper, along with the right to reproduce and to authorize others to reproduce all or part of the copyrighted paper. 
This document was prepared in conjunction with work accomplished under Contract No. DE-AC09-96SR18500 with the U. S. Department of Energy.

\section{DISCLAIMER}

This report was prepared as an account of work sponsored by an agency of the United States Government. Neither the United States Government nor any agency thereof, nor any of their employees, makes any warranty, express or implied, or assumes any legal liability or responsibility for the accuracy, completeness, or usefulness of any information, apparatus, product or process disclosed, or represents that its use would not infringe privately owned rights. Reference herein to any specific commercial product, process or service by trade name, trademark, manufacturer, or otherwise does not necessarily constitute or imply its endorsement, recommendation, or favoring by the United States Government or any agency thereof. The views and opinions of authors expressed herein do not necessarily state or reflect those of the United States Government or any agency thereof.

This report has been reproduced directly from the best available copy.

Available for sale to the public, in paper, from: U.S. Department of Commerce, National Technical Information Service, 5285 Port Royal Road, Springfield, VA 22161, phone: (800) 553-6847, fax: (703) 605-6900

email: orders@ ntis.fedworld.gov

online ordering: http://www.ntis.gov/support/index.html

Available electronically at http://www.osti.gov/bridge

Available for a processing fee to U.S. Department of Energy and its contractors, in paper, from: U.S. Department of Energy, Office of Scientific and Technical Information, P.O. Box 62, Oak Ridge, TN 37831-0062,

phone: (865)576-8401,

fax: (865)576-5728

email: $\underline{\text { reports@ adonis.osti.gov }}$ 
WSRC-TR-97-00289

Revision 1

February 14, 2002

Keywords:

NPH, Structural Evaluation, 105-L,

Reactors, L Disassembly Basin, Fuel

Storage, Seismic, Wind, Tornado

\section{A SUMMARY REPORT ON THE NPH EVALUATIN OF 105-L DISASSEMLBY BASIN (U)}

Prepared by:
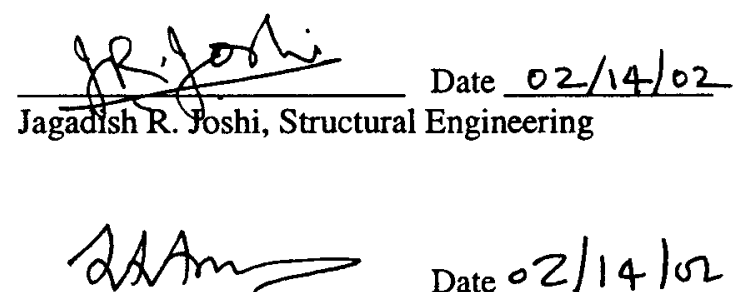

Date $02 / 14 / \Omega$

Jayprakash A. Amin, Structural Engineering

Technical Reviewer:

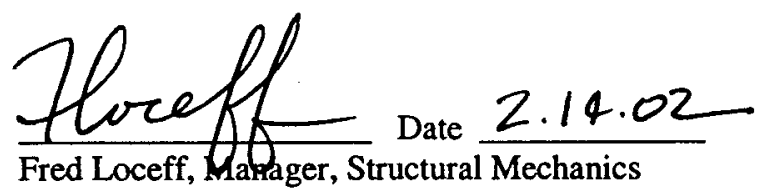

Approved by:

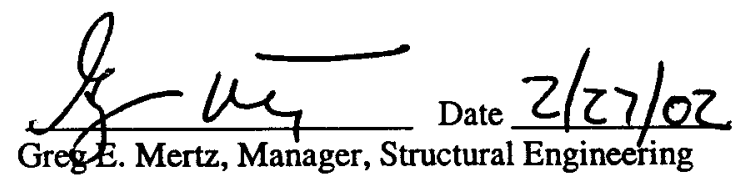

UNCLASSIFIED

Does Not Contain

Unclassified Controlled

Nuclear Information Material

Structural Engineering

PE \& C Division

Westinghouse Savannah River Company

Aiken, South Carolina

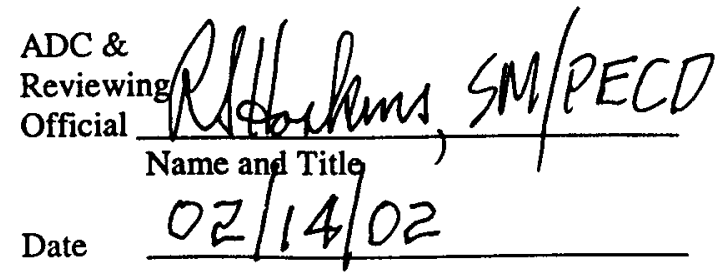




\section{SUMMARY}

The L Area Disassembly Basin (LDB) is evaluated for the natural phenomena hazards (NPH) effects due to earthquake, wind and tornado in accordance with DOE Order 420.1 and DOE-STD-1020. The deterministic analysis is performed for a Performance Category 3 (PC3) level of loads. Savannah River Site (SRS) specific NPH loads and design criteria are obtained from Engineering Standard 01060. It is demonstrated that the demand to capacity (D/C) ratios for primary and significant structural elements are acceptable (equal to or less than 1.0). Thus, 105-L Disassembly Basin building structure is qualified for the PC3 NPH effects in accordance with DOE Order 420.1 .

Credible II/I sources that are likely to cause seismic interaction with fuel stored in the horizontal bundle and bucket storage (HBBS) area in June 1997 were reviewed. The 5 ton Bridge Crane support system in the HBBS area requires modifications for handling the expected fuel weights. The proposed modifications to alleviate potential II over I interaction are incorporated in the determination of the D/C and fragility values for the crane support.

Based on a 2002 II/I evaluation update for the vertical tube storage (VTS) area it is concluded that the overall spacing between the stored fuel and the geometry of the fuel in bundles are not altered due to the potential impact from the VTS monorail support frame or from other falling objects.

A fragility analysis is performed to obtain the median capacities in terms of the peak ground acceleration (PGA) for seismic events, the fastest miles per hour (FMPH) for wind and tornado events, and the logarithmic standard deviations due to randomness and uncertainty. The minimum median PGA is $0.29 \mathrm{~g}$; it corresponds to a return period of about 20,000 years. 


\section{RECORD OF REVISION}

$\begin{array}{lll}\text { Revision \# } & \text { Description } & \text { Pages Affected } \\ 0 & \text { Initial Issue } & \text { All } \\ 1 & \begin{array}{l}\text { This revision resolves issues raised in Problem Identification Report } \\ \text { (PIR) No. 2001-PIR-09-010 and incorporates clarifications given in }\end{array} & \begin{array}{l}\text { pages 1 to 10, } \\ \text { "105-L Disassembly Basin - Vertical Tube Storage (VTS) } 3 \text { and 4 on }\end{array} \\ & \begin{array}{l}\text { Monorail Support Frame", IOM PEC-SEG-01-0042 of December 3, } 3 \text { and B4, } \\ \text { 2001, from G. E. Mertz to C. Nickell. }\end{array} & \begin{array}{l}\text { added page 2A } \\ \text { A 2002 II/I evaluation update on VTS and its conclusions are } \\ \text { included in this revision. }\end{array}\end{array}$




\section{TABLE OF CONTENTS}

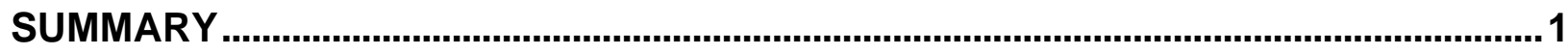

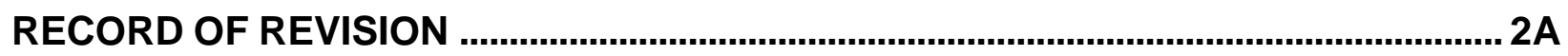

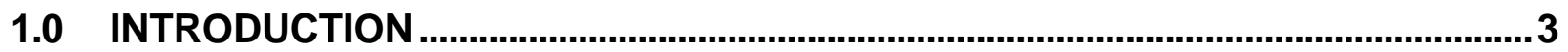

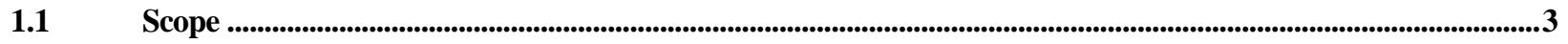

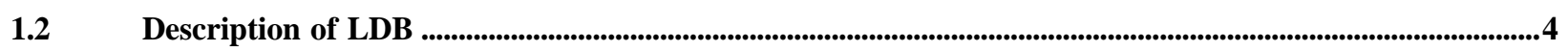

2.0 STRUCTURAL ANALYSIS AND ACCEPTANCE CRITERIA ................................4

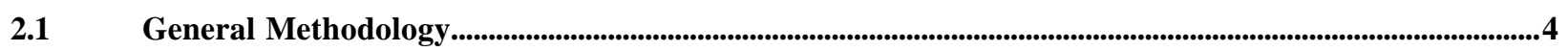

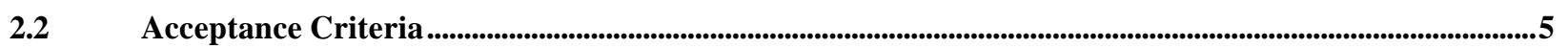

$2.3 \quad$ Soil Characteristics ..........................................................................................................................................................5

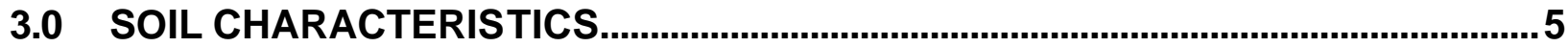

3.1 Lower Disassembly Basin and Transfer Area.................................................................................................................5

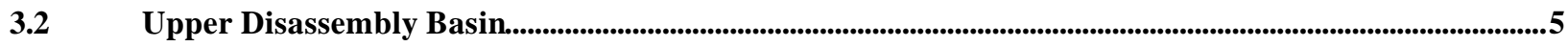

3.3 Multistick Model Parametric ............................................................................................................................5

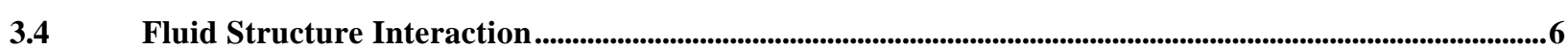

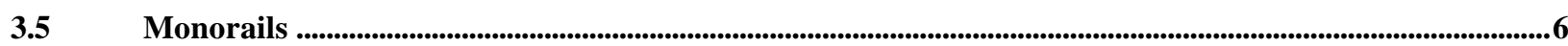

3.6 Piers, Walkways, and Roof...................................................................................................................................................6

4.0 FLOOR RESPONSE SPECTRA............................................................................

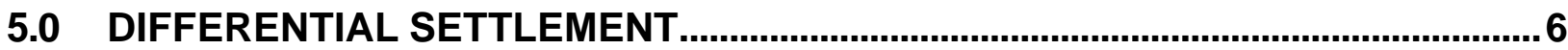

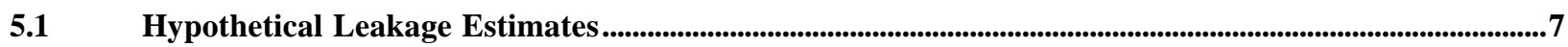

6.0 WIND AND TORNADO ANALYSIS .......................................................................... 7

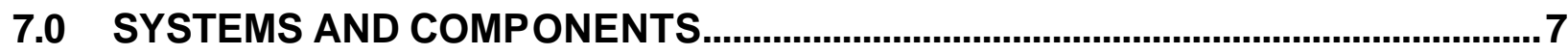

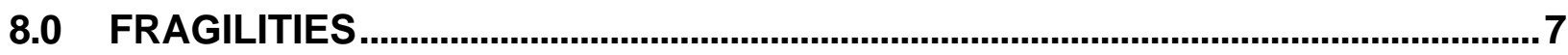

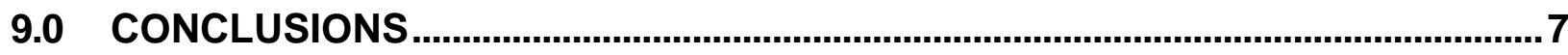

$9.1 \quad$ Demand to Capacity Ratios.............................................................................................................................................

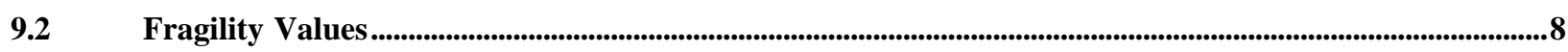

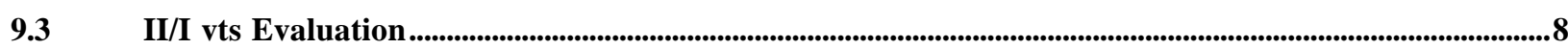

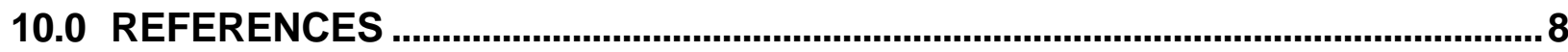

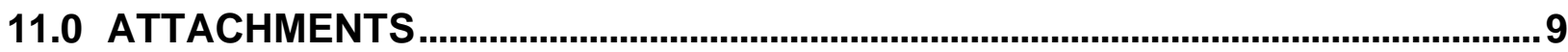




\subsection{INTRODUCTION}

The 105-L Disassembly Basin (LDB) is a sectionalized concrete basin containing about 3.4 million gallons of water in which irradiated fuel and target elements are stored. The basin serves to cool the irradiated fuel and to shield personnel from potential radiation. Fuel and target elements are handled under water from hangers attached to an overhead monorail system.

An assessment of typical 105-K Disassembly Basin, which is structurally similar to LDB, was made in February 1994 [1]. That assessment was related to the survivability of the basin walls and foundation mat based on an approximate estimate of its strength. A report on the status of the structural analysis of K, L and P Disassembly Basins, pertaining to the resistance to the natural phenomena hazards (NPH) effects, was prepared in December 1994 [2] subsequent to ceasing of NPH work on reactors.

A preliminary assessment of the roof, piers and walkways was provided in May 1996, [3], for the seismic survivability of these structural elements. It was concluded that modifications to these elements were not required for the no collapse criteria or to prevent II over I interaction.

A comprehensive structural analysis of safety related and structurally significant non-safety related structures, systems and components (SSC) in LDB was performed in FY97. This report presents a summary of the NPH analysis of LDB.

\subsection{SCOPE}

A brief description of the LDB is given in Section 1.2. It consists primarily of the Lower Disassembly Basin including Transfer Area, and the Upper Disassembly Basin Area.

The Fan Room located West of the Transfer Area is a light steel industrial type building and is included in the scope of this analysis to the extent its failure may cause a II over I interaction with the LDB.

Since a part of LDB is structurally integrated with the 105-L Process Building, the analysis of the LDB is required to verify that the results of the structural analysis of the Process Building, as presented in the summary report on 105K, [4] are not altered. Qualification of the 105-L Process building is based on its similarity to 105-K for which the Process Building analysis in Reference 4 was performed.

The LDB is analyzed for NPH effects from the design earthquake event at the Savannah River Site (SRS) considering the soil structure interaction (SSI) effects based on the dynamic soil properties given by Site Geotechnical Service (SGS). The LDB is analyzed for NPH effects due to design basis wind and tornado events at SRS. There are no safety related systems and components; however, systems and components that are likely to cause II over I interaction with the fuel stored in June 1997 are investigated through the original walkdowns of Reference 18. Additional 2002 walkdown effort is identified in the last paragraph of this section.

Credible II/I sources that could cause seismic interaction with the fuel stored in the horizontal bundle and bucket storage (HBBS) area were evaluated in June 1997. Not included in the scope of that walkdown [18] were issues related to the structural integrity of the monorail systems in the LDB. It is noted in interaction evaluation worksheets of Reference 18 that additional walkdowns would be required in Machine area, Emergency Disassembly area and Vertical Tube Storage area if fuel were to be stored in these areas in future.

Monorail systems in the Disassembly Basin Area are not evaluated in the walkdowns [18]. The Vertical Tube Storage (VTS) monorail support frame in 105-L Disassembly Area referenced in the Problem Identification Report, PIR No. 2001-PIR-09-010 is not included in the scope of this II/I walkdown evaluation. This was clarified in "105L Disassembly Basin - Vertical Tube Storage (VTS) Monorail Support Frame”, IOM PEC-SEG-01-0042 of December 3, 2001 from G. E. Mertz to C. Nickell. 
The II/I walkdown issues for the VTS in L area raised in the IOM of December 3, 2001, and in " $\mathrm{L}$ and $\mathrm{K}$ Disassembly Basin Suspended Monorail Loads", IOM PEC-SEG-01-0040, Revision 1 of January 21, 2002 from G. E. Mertz to C. Nickell are resolved in References 23 and 24. A 2002 II/I evaluation update of the VTS monorail frame and identification of all falling objects on fuel racks and stored fuel bundles in the VTS basin are provided in Reference 23. The effects of the falling objects on the fuel racks and the stored fuel bundles in VTS are determined in Reference 24. Conclusions of the 2002 II/I VTS evaluation update are given in Section 9.3.

\subsection{DESCRIPTION OF LDB}

A plan layout of the LDB is shown in Figure 1. It is divided into smaller interconnected water filled basins that include:

- Vertical Tube Storage (VTS)

- Machine Basin

- Horizontal Bundle and Bucket Storage (HBBS)

- Emergency Basin

- Transfer Bay

The VTS Area is in the Upper Disassembly Area, whereas, the rest of the basins are in the lower Disassembly Area. The later area is also sometimes referred to as the Lower Basin.

\subsection{STRUCTURAL ANALYSIS AND ACCEPTANCE CRITERIA}

\subsection{GenERAL METHODOLOGY}

The functional classification for LDB is found in Reference 6. The corresponding performance category is conservatively taken to be PC3. There are no safety related equipment or systems and components; however, selected equipment is evaluated for PC3 loads for determining the potential II over I interaction due to design basis NPH events. For the interaction purposes a "no collapse" criteria is used. Its objective is to assure that SSCs will not collapse and lead to a functional failure of structural elements or to criticality from a potential impact on fuel racks. The no collapse criteria does not require an adherence to code requirements.

The LDB is analyzed for vertical, lateral and hydrodynamic PC3 loads using fixed base and soil structure interact (SSI) models.

The seismic time history SSI analysis is performed for the lower bound, best estimate and upper bound (LB, BE and UB) soil properties using the impedance method as outlined in Section 3.3.4 of ASCE 4-86 [20]. Foundation impedance functions consisting of soil spring and damping coefficients are computed considering geometry and embedment effects. The coupled soil structure system is analyzed using the free-field surface motion as the control input motion. Fixed base response analysis is also performed for the free-field surface spectra input.

The combined response for the three earthquake components is obtained using the square root of the sum of the squares (SRSS) rule. Forces and displacements in structural elements are obtained by enveloping results from time history and response spectra analyses.

Parametric studies were performed for assessing the structure-soil-structure-interaction (SSSI) between the Process Building and Lower and Upper Disassembly Basins, and the potential pounding at the expansion joint between the Lower and Upper Disassembly Basins. 


\subsection{ACCEPTANCE CRITERIA}

SRS Engineering Standard 1060 [5] is used as the structural acceptance criteria for the structural evaluation of the LDB. Accordingly the 5 percent damping free field design response spectrum for performance category 3 (PC3) structures is used as the input in the seismic analysis. The input spectral coordinates are given in Table 1 and a plot of the spectrum in Figure 2.

Results are presented in terms of the demand to capacity (D/C) ratios for primary and other significant structural elements. Fragility values for the elements for the NPH effects are provided in terms of the median capacities and associated logarithmic variabilities.

\subsection{SOIL CHARACTERISTICS}

Preliminary dynamic soil properties for the LDB were given in July 1996 by Site Geotechnical Services (SGS) [7]. Confirmed soil properties became available in April 1997 [8]. These contained static and dynamic soil properties. The long term static displacement estimates in Reference 8 were slightly revised in Reference 9 when construction records of the building benchmarks became available. The dynamic displacements were provided in July 1997 by SGS [10].

Structural analysis of LDB is based on the soil properties given by SGS in References 8 through 10.

\subsection{SOIL CHARACTERISTICS}

Lower Disassembly and Upper Disassembly Areas are evaluated separately. The soil structure system is analyzed using the ABAQUS computer program using beam element (stick) modeling.

\subsection{LOWER DiSASSEMbly BASIN AND TranSFER AREA}

The model for the seismic analysis of the Lower Disassembly Basin and Transfer Area is shown in Figure 3. Details of the calculations, analysis and results are given in Volumes 1 and 2 of Reference 11.

\subsection{UPPER DISASSEMBLY BASIN}

The model for the seismic analysis of the Upper Disassembly Area (includes Process Building) is shown in Figure 4. Details of the calculations, analyses and results are given in Volumes 3 and 4 of Reference 11. The structural analysis for the 105-K Process Building (structurally identical to 105-L) was performed without including the Upper and Lower Disassembly Basins or the Transfer Area [4]. Analysis was performed to assess the impact of these Disassembly buildings on the Process Building [11, Vol. 3]. It is found that loads in structural elements of 105-L Process Building from the analysis of these models [11, Vol. 3] are less than or equal to those in Reference 4 for the corresponding members in $\mathrm{K}$.

\subsection{Multistick Model Parametric}

The multi-stick model for the seismic analysis of the combined Lower and Upper Disassembly Areas is shown in Figure 5. Details of the calculations, analysis and results are given in Reference12. This multi-stick model is used for parametric studies only; BE soil properties are used in the analysis. 


\subsection{FLUID STRUCTURE INTERACTION}

The Lower and Upper Basin walls are evaluated for the fluid structure interaction loads (sloshing of water in the basin) in Volume 1 of Reference 11 using the principles and procedures outlined in Reference 13.

\subsection{MONORAILS}

Revised D/C ratios for monorails in the Lower Basin Areas were computed in Reference 14 for various fuel weights. This evaluation is based on prior calculations and reports on factors of safety, and on the test data for anchorage inserts at SRS reactors ("Factor of Safety Report for Monorail Modification for Assembly/Disassembly Areas in P, K \& L Reactor Buildings", UE\&C, July 25, 1990, "Design Engineering Report on the Evaluation of Monorail Upgrades in the VTS and Non-VTS Areas P, K and L Reactors", July 1990, and "Upgrade of Assembly/Disassembly Monorails”, RTR 2366, from W. E. Mayo and H. D. Kane to G. G. Merz, June 29, 1987).

The monorail support frame in the Emergency Basin was evaluated in Reference 15. An occasional fuel load of 200 pounds was considered in the analysis [15].

The weight of the monorail support frame in the VTS and a maximum monorail fuel load of 200 pounds were considered in the structural evaluation of the walkways and piers in the VTS [15]. The VTS monorails and the monorail support frame were not evaluated in the calculation.

\subsection{PIERS, WALKWAYS, AND ROOF}

A preliminary assessment of the roof, piers and walkways was provided in May 1997 [3], for the seismic survivability of the structural elements. The conclusions of this assessment were confirmed by a more detailed analysis [15]. Piers and walkways do not satisfy code requirements, however, it was determined that they would not fail or collapse causing a II over I interaction for the design basis seismic event.

\subsection{FLOOR RESPONSE SPECTRA}

The in-structure amplified floor response spectra (FRS) for the Lower Disassembly Area and Transfer Area are obtained from the model given in Section 3.1. SSI analyses using the LB, BE and UB soil properties are performed, and spectra are developed by enveloping and broadening in accordance with procedures given in ASCE 4-86 [20] as invoked by Site Standards [5].

Similarly FRS for the Upper Disassembly Area are obtained form the model given in Section 3.2.

The spectra are generated for damping ratios of 4, 5, 7 and 10 percent for elevations $-30.0,-17.0,+15.0$ and +40.0 feet, as applicable, since these elevations do not exist in all structures. These spectra and digitized values are provided in Attachment $\mathrm{C}$ of Section 11.

\subsection{DIFFERENTIAL SETTLEMENT}

The LDB is evaluated for differential displacements provided by SGS as detailed in Section 2.3. The evaluation is done for static and dynamic settlements [16]. The settlements provided by SGS do not explicitly account for the stiffness of the structure. The structural evaluation of LDB for differential settlements is based on a method that neglects the stiffness of the foundation mat, which is conservative, compared to methods that consider the stiffness. It was determined that the differential settlements may lead to non-through wall and foundation mat cracking due to bending; that is, the compression side of the structural element loaded in bending would maintain a leak tight configuration, at the junction of the mat with the external structural walls. 


\subsection{HYPOTHETICAL LEAKAGE ESTIMATES}

Even though neither the foundation mat nor the basin walls are expected to develop through thickness cracks, which allow leakage of water out of the disassembly basin, estimates are made [17] of the hypothetical leakage through various uniform widths of cracks in the LDB at the maximum height of water of 30 feet. The results of the parametric basin drawdown analysis are summarized in Table 2.

\subsection{WIND AND TORNADO ANALYSIS}

The LDB is analyzed for wind and tornado effects in [14]. By comparison of the total lateral shear resulting from the wind and tornado loads with those from an earthquake, D/C ratios for the primary shear walls for the wind and tornado events are found. They are significantly smaller than 1.0. Thickness of concrete walls and the roof is large enough to resist the penetration and potential interaction effects of missiles stipulated in DOE-STD-1020 as invoked by Reference 5 .

\subsection{SYSTEMS AND COMPONENTS}

Seismic Qualification Utility Group (SQUG) walkdown methodology is used to review credible II/I sources that are likely to cause seismic interaction with the fuel stored in HBBS area in June 1997 [18]. See Section 1.1 for clarification of the scope of the walkdowns. It is determined that except for the 5 ton crane in HBBS area, the systems and components in LDB do not present a seismic interaction hazard to the storage racks. The 5 ton crane support system requires modifications which are incorporated in the results presented in this report.

Conclusions of the 2002 II/I VTS evaluation update are given in Section 9.3.

\subsection{FRAGILITIES}

Fragility estimates for the primary and other significant structural elements of the LDB are provided in Reference 19. The fragilities are based on the deterministic analysis done for the design basis events and on the expected variabilities. The median capacities are given in terms of the peak ground acceleration (PGA) for seismic events, and the fastest miles per hour (FMPH) for wind and tornado events. High confidence of a low probability of failure (HCLPF) values for the structural elements are also provided.

\subsection{CONCLUSIONS}

Based on the analysis of L Area Disassembly Basin (LDB) for the NPH effects due to PC3 earthquake, wind and tornado in accordance with DOE Order 420.1 and DO-STD-1020, it is determined that the D/C ratios for primary and other significant structural elements are equal to or less than 1.0. Thus 105-L Disassembly Basin is qualified for the PC3 NPH effects in accordance with DOE Order 420.1.

\subsection{DEMAND To CAPACITY RATIOS}

A summary of the D/C ratios for the safety related and structurally significant elements of the LDB is given in Table. 3. 


\subsection{Fragility VALUES}

Fragility values for the safety related and structurally significant elements of the LDB are given in Table 4. These may be used for safety analysis or for beyond design basis evaluations. The minimum PGA for the structural elements is $0.29 \mathrm{~g}$ [19]; this corresponds to a return period of about 20,000 years or an annual exceedance probability at 5E-5 [21]. The median FMPH for the structure is 413 miles per hour [19]; this corresponds to a return period of over 1E7 years or an annual exceedance probability less than 1E-7 [22].

\section{$9.3 \quad$ II/I VTS EVALUATION}

Conclusions of the 2002 II/I VTS evaluation update are given here. Reference 23 demonstrates that both the walkway and pier are capable of resisting the impact forces from the collapse of the VTS monorail frame and the failed monorail support frame will come to rest on the platform. The effect of the falling objects identified in Reference 23 on the stored fuel and fuel racks in the VTS is determined in Reference 24. It is concluded that the overall spacing between stored fuel and the geometry of fuel in bundles are not altered by the impact from the potential missiles.

\subsection{REFERENCES}

1. “Assessment of 105-K Disassembly Basin”, IOM 730:EPD-SE-94-0054 of February 15, 1994 from M.E. Maryak to T.C. Esterling.

2. "Status Report on Structural Analysis of K, L and P Disassembly Areas (U)", IOM ECS-CES-94-0274 of December 19, 1994 from J.R. Joshi to J.C. Guy.

3. "Assessment of Seismic Qualification of Concrete Piers, Walkways and Roof for L Disassembly Area (U)", IOM ECS-CES-96-0021 of May 14, 1996 from R.L. Bandyopadhyay and J.R. Joshi to S. Pogula.

4. "Summary Report on the Seismic Qualification of the 105-K Reactor Building (U)", WSRC-TR-91-604, Revision 2, May 1992.

5. "Structural Design Criteria”, Engineering Standard 01060, Revision 1, October 1996, WSRC Manual WSRCTM-95-1.

6. "Functional Classification Report for L Reactor Facility", WSRC-TR-95-0399, Revision 1, June 1997.

7. "Selected Soil Properties for L-Area Dynamic Profile", IOM ECSD-SGS-96-228 of July 3, 1996 from Walt Rabe to J.R. Joshi.

8. "Confirmed Soil Properties for the 105-L Disassembly Area:, IOM PECD-SGS-97-134 of April 25, 1997 from Todd E. Ross to J.R. Joshi.

9. “Long Term Settlement Estimation for the L Disassembly Basin", cc:Mail of May 15, 1997 from Todd Ross to J.R. Joshi.

10. "Liquefaction Potential and Settlement Estimates, 105-L Disassembly Basin", IOM PECD-SGS-97-0187 of July 9, 1997 from Michael D. McHood to J.R. Joshi.

11. "NPH Evaluation of 105-L Disassembly Basin", T-CLC-L-00006, Revision 1, Volumes 1 to 4, Structural Engineering, 2002.

Volume 1: Lower Disassembly Basin \& Transfer Area

Volume 2: Lower Disassembly Basin \& Transfer Area, Computer Runs - ABAQUS Input/Output

Volume 3: Upper Disassembly Basin

Volume 4: Upper Disassembly Basin, Computer Runs - ABAQUS Input/Output

12. "105-L Multistick Model Parametric Study-Disassembly Basin", T-CLC-L-00013, Structural Engineering, 1997.

13. "Seismic Design and Evaluation Guidelines for the DOE High-Level Waste Storage Tanks and Appurtenances", K. Bandyopadhyay, A. Cornell, C. Costantino, R. Kennedy, C. Miller and A. Veletsos, BNL 52361, Rev. 10/95, UC-406, UC-510, October 1995.

14. "Miscellaneous Technical Issues in 105-L Structural Analysis", T-CLC-L-00017, Revision 1, Structural Engineering, 2002. 
15. "Disassembly Area Building, Analysis of Piers, Walkway and Roof of 105-L Disassembly Basin", T-CLC-L00003, Revision 1, Structural Engineering, 2002.

16. “105-L Disassembly Basin Differential Settlement Analysis”, T-CLC-L-00012, Structural Engineering, 1997.

17. "Postulated Seepage Rates form 105-L Basin", T-CLC-L-00014, Structural Engineering \& SGS, 1997.

18. "Seismic II/I Interaction for 105-L Disassembly Area", T-CLC-L-00016, Revision 3, Structural Engineering, 2002.

19. "Fragility Analysis of 105-L Disassembly Basin Structure", T-CLC-L-00018, Revision 1, Structural Engineering, 2002.

20. "Seismic Analysis of Safety-Related nuclear Structures and Commentary on Standard for Seismic Analysis of Safety-Related Nuclear Structures", ASCE Standard 4-86, 1987.

21. "Development of probabilistic Seismic Hazard Curve for DWPF at SRS (U)", WSRC-TR-93-317, Revision 1, January 1995, H.E.Wingo.

22. "Natural Phenomena Hazards Modeling Project: Extreme Wind/Tornado Models for the Department of Energy Sites", By D.W. Coats and R.C. Murray, UCRL-53526, Revision 1, August 1985.

23. "L-Area Disassembly Basin Vertical Tube Storage II/I Evaluation”, Engineering Calculation, T-CLC-L-00046, Structural Engineering, 2002.

24. "VTS Fuel Racks II/I Analysis (U)”, Engineering Calculation, T-CLC-L-00045, Structural Mechanics, 2002.

\subsection{ATTACHMENTS}

\section{A List of Figures}

Figure 1

Figure 2

Figure 3

Figure 4

Figure 5
Plan Layout of 105-L Disassembly Area PC3 Design Response Spectra, 5 Percent Damping, Horizontal and Vertical SSI Model for Lower disassembly and Transfer Area SSI Model for Upper Disassembly Area and Process Building SSI Model for Combined Lower and Upper Disassembly Areas and Process Building

\section{B List of Tables}

Table 1

Coordinates for PC3 Design Response Spectra, 5 Percent Damping, Horizontal and Vertical

Table 2

Summary of Parametric L Basin Drawdown

Table 3

Significant Demand to Capacity Ratios for L Disassembly Basin

Table 4

\section{Floor Response Spectra}

Lower Disassembly Covers Column Line 106 to 111

$\begin{array}{lll}\text { Figure C1 } & \text { N-S Direction Response Spectra } & \text { Elev. }-17, \\ \text { Figure C1 } & \text { N-S Direction Digitized Values } & \text { Elev. }-17, \\ \text { Figure C2 } & \text { N-S Direction Response Spectra } & \text { Elev. +15, } \\ \text { Figure C2 } & \text { N-S Direction Digitized Values } & \text { Elev. +15, } \\ \text { Figure C3 } & \text { E-W Direction Response Spectra } & \text { Elev. }-17, \\ \text { Figure C3 } & \text { E-W Direction Digitized Values } & \text { Elev. -17, } \\ \text { Figure C4 } & \text { E-W Direction Response Spectra } & \text { Elev. -15, } \\ \text { Figure C4 } & \text { E-W Direction Digitized Values } & \text { Elev. }-15 \\ \text { Figure C5 } & \text { Vertical Direction Response Spectra } & \text { Elev. +15, } \\ \text { Figure C5 } & \text { Vertical Direction Digitized Values } & \text { Elev. +15, }\end{array}$


Upper Disassembly Area Covers Column Line 101 to 106

Figure C6

Table C6

Figure $\mathrm{C} 7$

Table C7

Figure $\mathrm{C} 8$

Table C8

Figure C9

Table C9

Figure $\mathrm{C} 10$

Table C10
N-S Direction Response Spectra

N-S Direction Digitized Values

N-S Direction Response Spectra

N-S Direction Digitized Values

E-W Direction Response Spectra

E-W Direction Digitized Values

E-W Direction Response Spectra

E-W Direction Digitized Values

Vertical Direction Response Spectra

Vertical Direction Digitized Values
Elev. -30 "

Elev. -30 ,

Elev. +15 ,

Elev. +15 ,

Elev. -30 '

Elev. -30 '

Elev. +15 ,

Elev. +15 ,

Elev. +15 ,

Elev. +15 ,

\section{Transfer Area Covers Column Line 111 to 112}

Figure $\mathrm{C} 11$

Table C11

Figure $\mathrm{C} 12$

Table $\mathrm{C} 12$

Figure $\mathrm{C} 13$

Table C13

Figure $\mathrm{C} 14$

Table C14

Figure $\mathrm{C} 15$

Table C15
N-S Direction Response Spectra

N-S Direction Digitized Values

N-S Direction Response Spectra

N-S Direction Digitized Values

E-W Direction Response Spectra

E-W Direction Digitized Values

E-W Direction Response Spectra

E-W Direction Digitized Values

Vertical Direction Response Spectra

Vertical Direction Digitized Values
Elev. -17 ,

Elev. -17 '

Elev. +40 ,

Elev. +40 '

Elev. -17'

Elev. -17'

Elev. +40 ,

Elev. +40 '

Elev. +40 '

Elev. +40 ' 


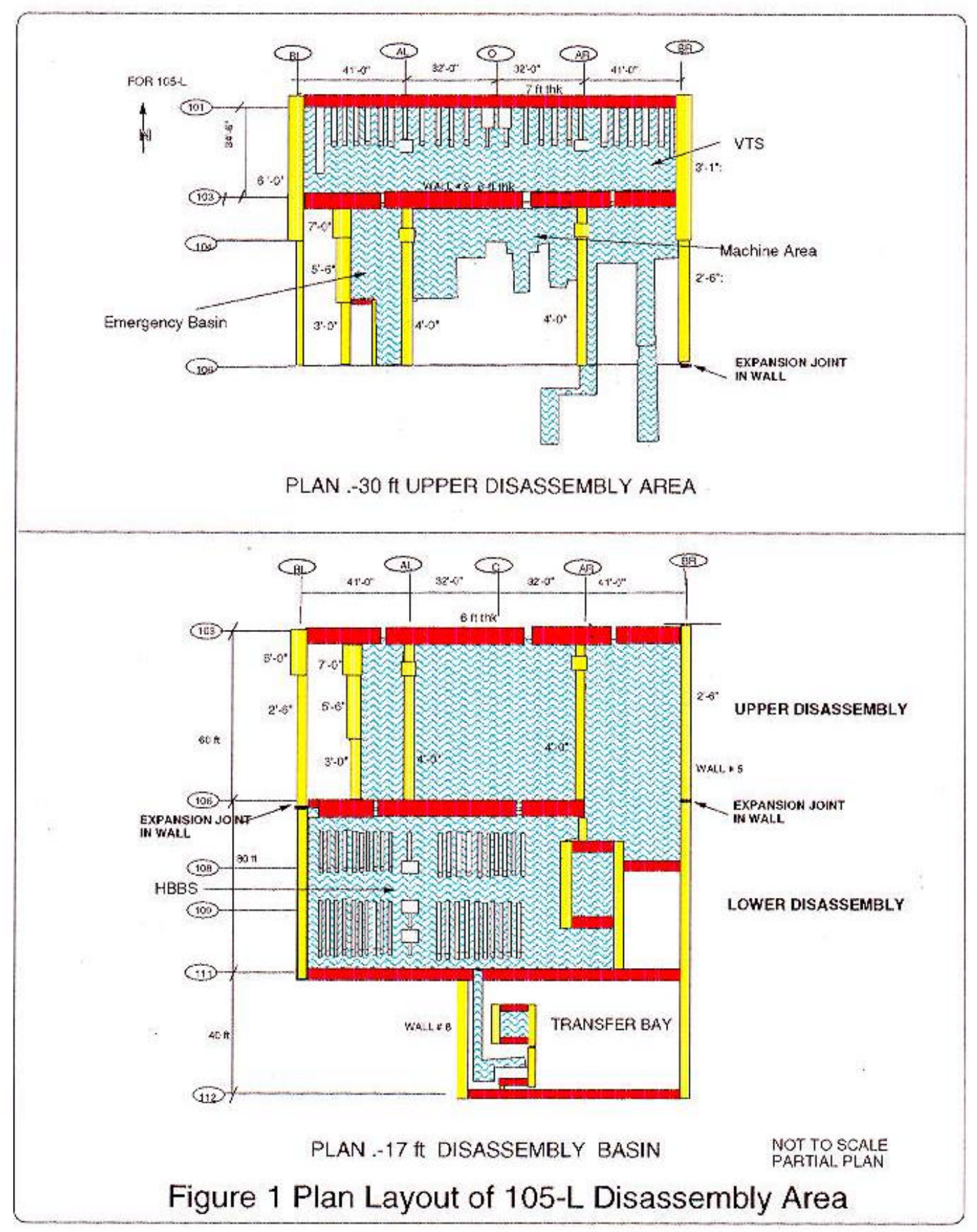




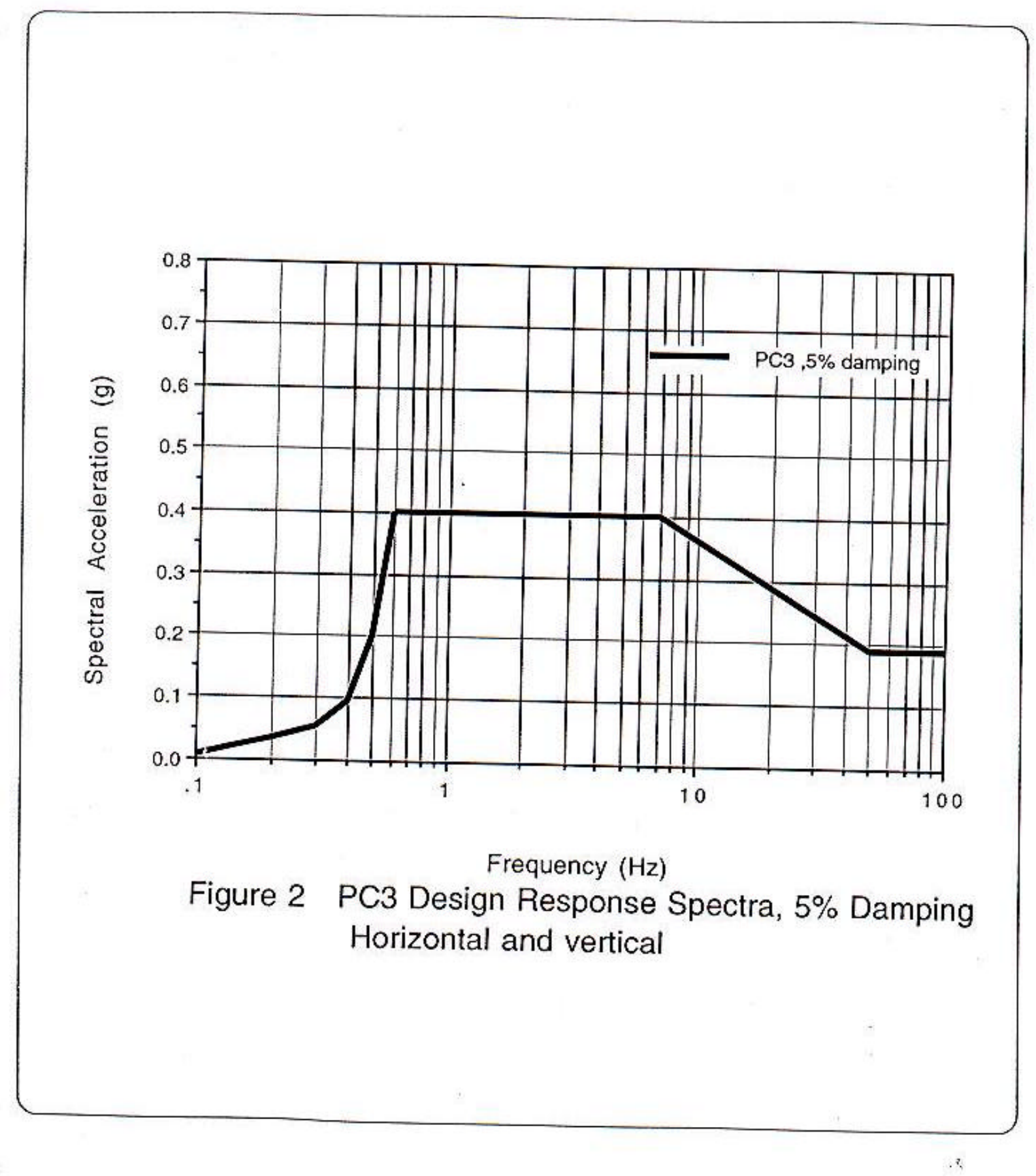




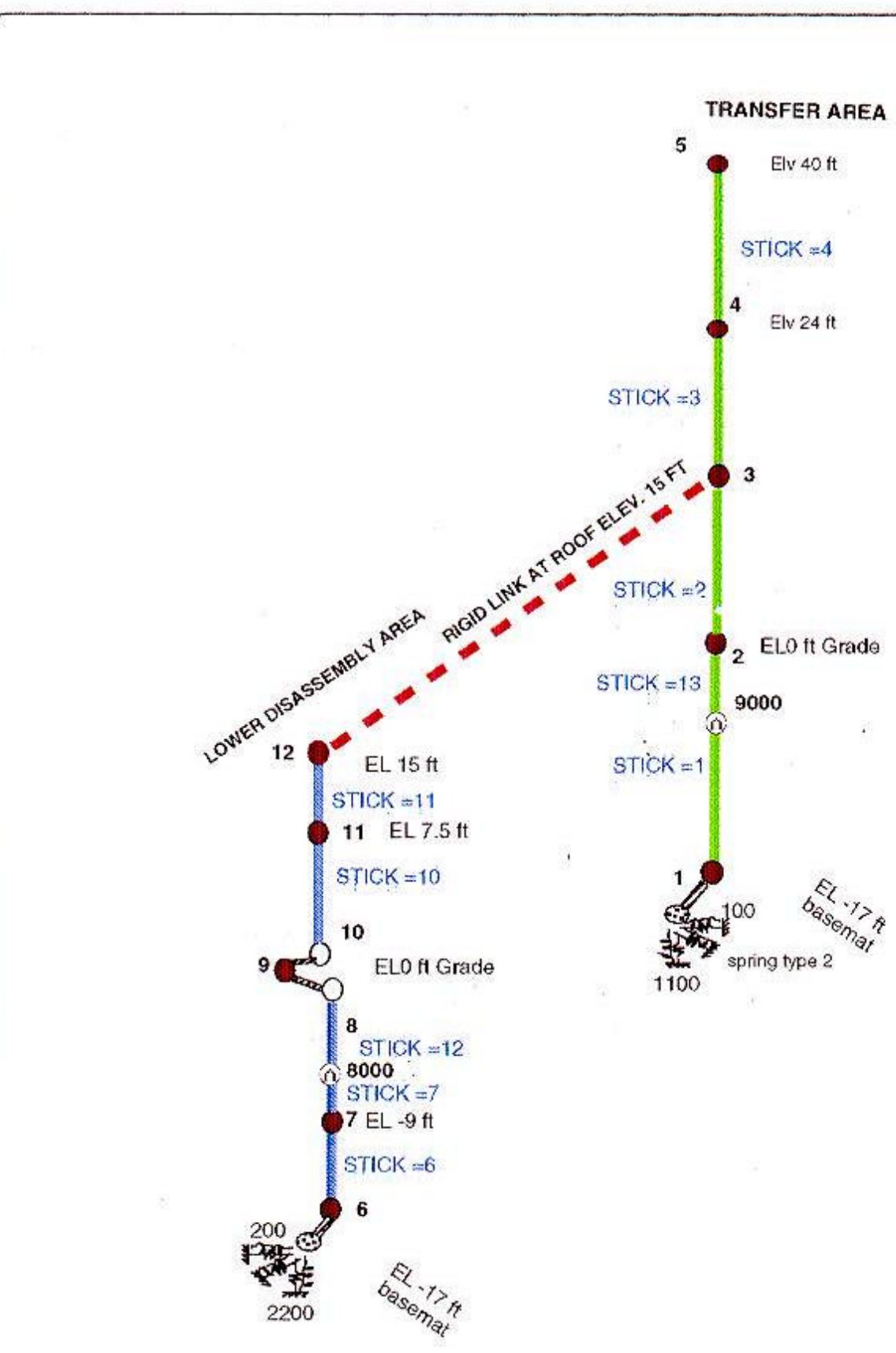

Figure 3 SSI Model for 105-L Lower Disassembly area and Transfer Area 
WSRC-TR-97-00289

Revision 0

Page A4 of A5

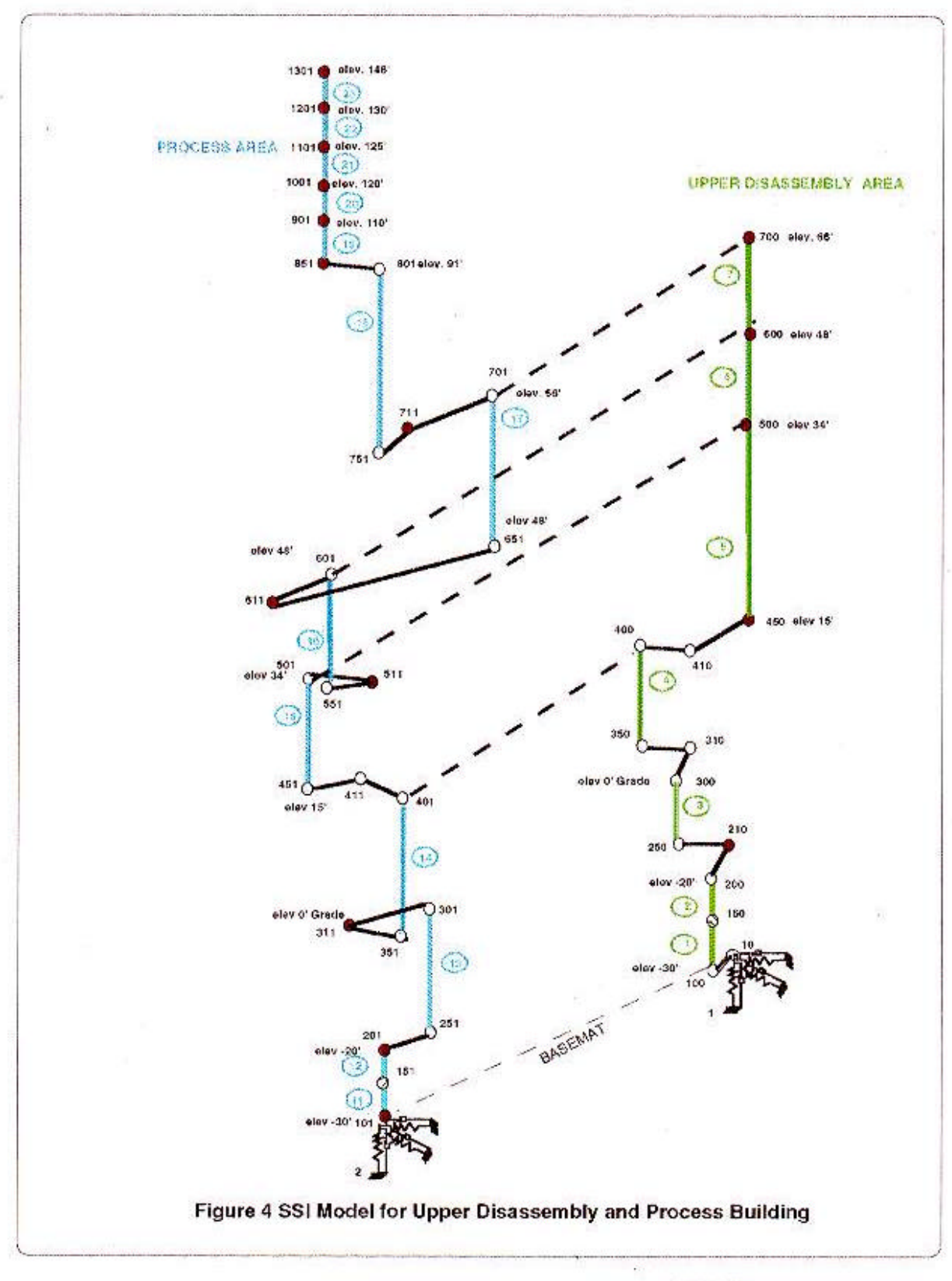

HeT TO SCGLE 


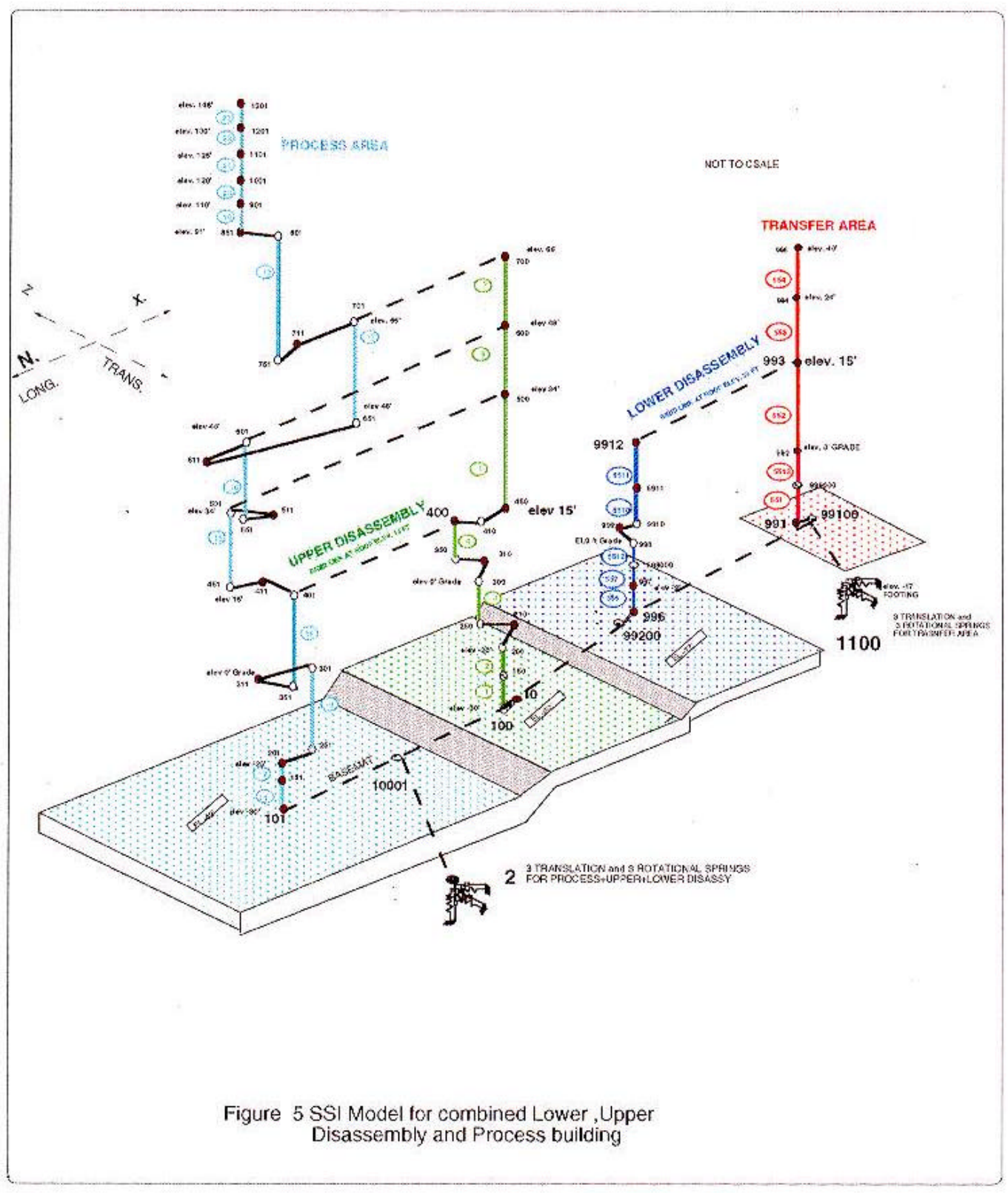

Not es scaia 
WSRC-TR-97-00289

Revision 0

Page B1 of B4

\begin{tabular}{|c|c|}
\hline Frequency $(\mathrm{Hz})$ & Spectral Acceleration (g) \\
\hline \hline 0.100 & 0.006 \\
\hline 0.200 & 0.033 \\
\hline 0.300 & 0.054 \\
\hline 0.400 & 0.096 \\
\hline 0.500 & 0.202 \\
\hline 0.600 & 0.400 \\
\hline 7.000 & 0.400 \\
\hline 50.000 & 0.190 \\
\hline 100.000 & 0.190 \\
\hline
\end{tabular}

Digitize values for Figure 2

TABLE 1: Coordinates for PC3 Design Response Spectra, 5 Percent Damping Horizontal and Vertical 
105-L Disassembly BASIN (30 ft Depth)

\begin{tabular}{|c|c|c|c|c|c|c|}
\hline TABLE \# & Crack Inch & $\begin{array}{c}\mathrm{Kh} \\
\mathrm{cm} / \mathrm{s}\end{array}$ & $\begin{array}{c}\mathrm{Kv} \\
\mathrm{cm} / \mathrm{s}\end{array}$ & $\mathrm{Kh} / \mathrm{Kv}$ & $\begin{array}{c}\text { WATER } \\
\text { LEVEL } \\
\text { DROP (IN) }\end{array}$ & $\begin{array}{c}\text { TIME } \\
\text { TAKES IN } \\
\text { DAYS } \\
\end{array}$ \\
\hline 1 & $1 / 16$ in & $5,00 \mathrm{E}-04$ & $1.00 \mathrm{E}-04$ & 5 & 12 in & 126 \\
\hline $1 \mathrm{~A}$ & $1 / 2$ in & 5.00 E-04 & $1.00 \mathrm{E}-04$ & 5 & 12 in & 100 \\
\hline IB & 1 in & $5.00 \mathrm{E}-04$ & $1.00 \mathrm{E}-04$ & 5 & 12 in & 91.5 \\
\hline $1 \mathrm{C}$ & 3 in & $5.00 \mathrm{E}-04$ & $1.00 \mathrm{E}-04$ & 5 & 12 in & 77.9 \\
\hline 2 & $1 / 16$ & $1.00 \mathrm{E}-03$ & $1.00 \mathrm{E}-04$ & 10 & 12 in & 65 \\
\hline $2 A$ & $1 / 2$ in & $1.00 \mathrm{E}-03$ & $1.00 \mathrm{E}-04$ & 10 & 12 in & 52.1 \\
\hline 2B & 1 in & $1.00 \mathrm{E}-03$ & $1.00 \mathrm{E}-04$ & . 10 & 12 in & 47.8 \\
\hline $2 \mathrm{C}$ & 3 in & $1.00 \mathrm{E}-04$ & $1.00 \mathrm{E}-04$ & 10 & 12 in & 41 \\
\hline 3 & $1 / 16$ in & $5.00 \mathrm{E}-03$ & $1.00 \mathrm{E}-04$ & 50 & 12 in & 14 \\
\hline $3 \mathrm{~A}$ & $1 / 2$ in & $5.00 \mathrm{E}-03$ & $1.00 \mathrm{E}-04$ & 50 & 12 in & 11.4 \\
\hline $3 \mathrm{~B}$ & $1 \mathrm{in}$ & $5.00 \mathrm{E}-03$ & $1.00 \mathrm{E}-04$ & 50 & 12 in & 10.6 \\
\hline $3 C$ & 3 in & $5.00 \mathrm{E}-03$ & $1.00 \mathrm{E}-04$ & 50 & $12 \mathrm{in}$ & 9.2 \\
\hline
\end{tabular}

TABLE 2: Summary of Parametric L Basin Drawdown 


\begin{tabular}{|c|c|c|c|c|c|}
\hline ITEM\# & DESCRIPTION & \multicolumn{4}{|l|}{ SEISMIC } \\
\hline & & $\begin{array}{l}\text { Reference } \\
\text { Calc.\# T- } \\
\text { CLC-L-000 }\end{array}$ & Page \# & Comment & $\mathrm{D} / \mathrm{C}$ \\
\hline 1 & $\begin{array}{l}\text { Primary Shear Walls } \\
\text { Upper Disassembly Area } \\
\text { Lower Disassembly Area } \\
\text { Transfer Area } \\
\text { Pressure on External Walls } \\
\text { with water (Lower Disassembly) } \\
\text { without water (Lower Disassembly) }\end{array}$ & $\begin{array}{l}06, \text { v. } 3 \\
06, \text { v. } 1 \\
06, \text { v. } 1 \\
06, \text { v. } 1 \\
06, \text { v. } 1\end{array}$ & $\begin{array}{l}75 \\
11 \\
11 \\
\\
\\
11 \\
11\end{array}$ & & $\begin{array}{l}0.29 \\
0.22 \\
0.35 \\
\\
\\
\\
0.74 \\
0.97 \\
\end{array}$ \\
\hline 2 & $\begin{array}{l}\text { Structural Columns ( } 4 \text { X } 4 \text { and } 6 \text { X 6) } \\
4 \text { X } 4 \text { at Col. AAL \& } 102\end{array}$ & 06, v. 3 & 75 & $\begin{array}{l}\text { Upper Dissassy, } \\
\text { axial + bend. }\end{array}$ & 0.83 \\
\hline 3 & Roof (Span: 63 and 45) & 03 & 26 & & 0.85 \\
\hline 4 & $\begin{array}{l}\text { Foundation Mat } \\
\text { Lower Disassembly }\end{array}$ & 12 & 8 & $\begin{array}{l}\text { differential } \\
\text { settlement. }\end{array}$ & 0.76 \\
\hline 5 & $\begin{array}{l}\text { VTS (maximum fuel load of } 200 \text { pounds) } \\
\text { Piers } \\
\text { Concrete Beam/Walkway }\end{array}$ & $\begin{array}{l}03 \\
03\end{array}$ & $\begin{array}{l}29 \\
25\end{array}$ & & $\begin{array}{l}0.93 \\
0.88\end{array}$ \\
\hline 6 & $\begin{array}{l}\text { Emergency Basin (occasional fuel load of } \\
200 \text { pounds) } \\
\quad \text { Piers } \\
\quad \text { Concrete Beam/Walkway } \\
\end{array}$ & $\begin{array}{l}03 \\
03 \\
\end{array}$ & $\begin{array}{l}29 \\
25 \\
\end{array}$ & & $\begin{array}{l}0.93 \\
1.0 \\
\end{array}$ \\
\hline 7 & $\begin{array}{l}\text { Machine Basin and HBBS (Lower Basin) } \\
\text { Piers } \\
\text { Concrete Beams/Walkway }\end{array}$ & $\begin{array}{l}03 \\
03 \\
\end{array}$ & $\begin{array}{l}25 \\
25 \\
\end{array}$ & & $\begin{array}{l}1.0 \\
0.21 \\
\end{array}$ \\
\hline 8 & $\begin{array}{l}\text { Monorail } \\
\text { HBBS (non-Piers : fuel: } 495 \text { lb.) } \\
\text { Machine Basin (non-Piers : fuel: } 495 \text { lb.) }\end{array}$ & $\begin{array}{l}17 \\
17\end{array}$ & $\begin{array}{l}11 \\
11\end{array}$ & & $\begin{array}{l}0.26 \\
0.23\end{array}$ \\
\hline ITEM\# & DESCRIPTION & \multicolumn{4}{|c|}{ WIND/TORNADO } \\
\hline 1 & $\begin{array}{c}\text { Primary Structural Walls } \\
\text { Lower Disassembly }\end{array}$ & 06, v. 1 & $305 \mathrm{C}$ & & 0.11 \\
\hline
\end{tabular}

TABLE 3: Significant Demand to Capacity Ratios for L Disassembly Basin 


\begin{tabular}{|c|c|c|c|c|c|}
\hline & DESCRIPTION & FRAGII & & & \\
\hline ITEM \# & SEISMIC & $\begin{array}{l}\text { Median } \\
\text { PGA,g }\end{array}$ & $\mathbf{b}_{\mathbf{R}}$ & $\mathbf{b}_{\mathbf{U}}$ & $\begin{array}{l}\text { HCLPF } \\
\text { PGA,g }\end{array}$ \\
\hline 1 & $\begin{array}{l}\text { Primary Shear Walls } \\
\text { Upper Disassembly Area } \\
\text { Lower Disassembly Area } \\
\text { Transfer Area } \\
\text { Pressure on External Walls } \\
\text { with water (Lower Disassembly) } \\
\text { without water (Lower Disassembly) }\end{array}$ & $\begin{array}{l}1.28 \\
1.07 \\
\\
0.72 \\
0.52 \\
\end{array}$ & $\begin{array}{l}0.31 \\
0.31 \\
\\
0.31 \\
0.31 \\
\end{array}$ & $\begin{array}{l}0.4 \\
0.4 \\
\\
0.41 \\
0.41\end{array}$ & $\begin{array}{l}0.40 \\
0.33 \\
0.22 \\
0.16\end{array}$ \\
\hline 2 & Structural Columns (4 X 4 and 6 X 6) & 0.52 & 0.31 & 0.41 & 0.16 \\
\hline 3 & Roof (Span: 63 and 45) & 0.51 & 0.31 & 0.41 & 0.15 \\
\hline 4 & $\begin{array}{l}\text { Foundation Mat } \\
\text { Lower Disassembly }\end{array}$ & 0.57 & 0.31 & 0.41 & 0.17 \\
\hline 5 & $\begin{array}{l}\text { VTS (maximum fuel load of } 200 \text { pounds) } \\
\text { Piers }\end{array}$ & 0.31 & 0.31 & 0.41 & 0.09 \\
\hline 6 & $\begin{array}{l}\text { Emergency Basin (occasional fuel load of } 200 \text { pounds) } \\
\text { Piers }\end{array}$ & 0.51 & 0.31 & 0.41 & 0.15 \\
\hline 7 & $\begin{array}{l}\text { Machine Basin and HBBS (Lower Basin) } \\
\text { Piers }\end{array}$ & 0.29 & 0.31 & 0.41 & 0.09 \\
\hline 8 & $\begin{array}{l}\text { Monorail } \\
\text { HBBS (non-Piers, fuel: } 495 \text { pounds) } \\
\text { Machine Basin (non-Piers, fuel: } 495 \text { pounds) }\end{array}$ & $\begin{array}{l}0.70 \\
0.73\end{array}$ & $\begin{array}{l}0.34 \\
0.34\end{array}$ & $\begin{array}{l}0.31 \\
0.31\end{array}$ & $\begin{array}{l}0.24 \\
0.25\end{array}$ \\
\hline ITEM\# & WIND/TORNADO & $\begin{array}{l}\text { Median } \\
\text { MPH }\end{array}$ & $\mathbf{b}_{\mathbf{R}}$ & $\mathbf{b}_{\mathrm{U}}$ & $\begin{array}{l}\text { HCLPF } \\
\text { MPH }\end{array}$ \\
\hline 1 & $\begin{array}{c}\text { Primary Structural Walls } \\
\text { Lower Disassembly }\end{array}$ & 413 & 0.2 & 0.2 & 213 \\
\hline
\end{tabular}

NOTES:

PGA:

MPH:

$\mathrm{b}_{\mathrm{R}}$ :

Peak Ground Acceleration

$\mathrm{b}_{\mathrm{U}}:$
$\mathrm{HCLPF}$

Fastest Miles Per Hour wind/tornado speed

Logarithmic Standard Deviation for Randomness

Logarithmic Standard Deviation for Uncertainty

High Confidence Low Probability of Failure

TABLE 4: Significant Fragility Values for L Disassembly Basin 
FIGURE C-1

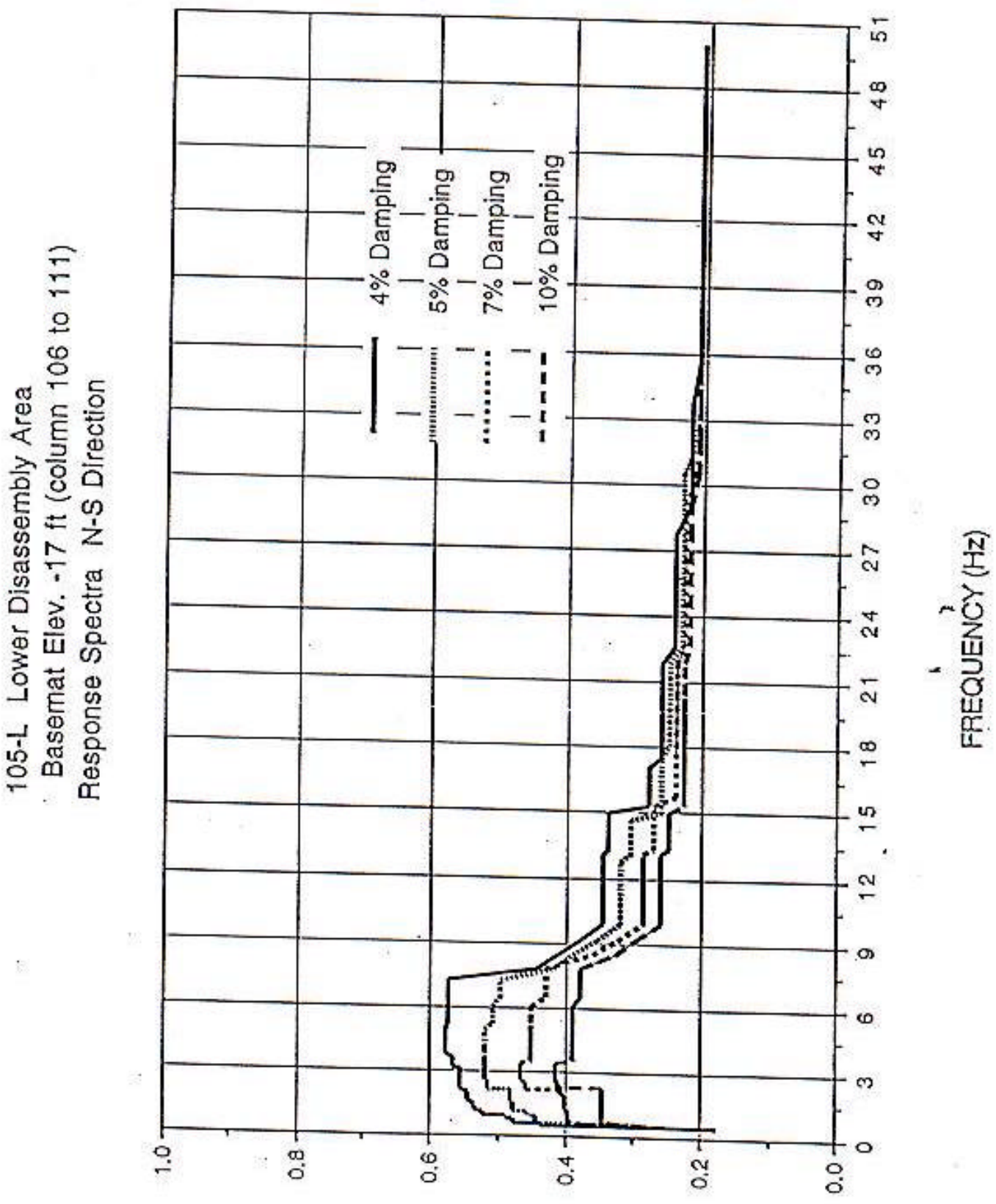

(6) NO11VHA73OOV 
WSRC-TR-97-00289

Revision 0

Page C2 of C30

TABLE C-I

105-L LOWER DISASSEMBL, $Y$ AAEA (ColuTM 106 to 111)

Elov. -17 II BAsornat AESPONSE SPECTRA

N-S DIAECTON

\begin{tabular}{|c|c|c|c|c|}
\hline $\begin{array}{c}\text { FEQDENCY } \\
(k\langle x)\end{array}$ & 1\%. DAMPNG & $\begin{array}{l}\text { CERERATKON } \\
\text { SXONAPANG }\end{array}$ & $\begin{array}{l}\text { (9) } \\
\text { rX DANAPRG }\end{array}$ & $10 \%$ OALAPUNG \\
\hline 0.500 & 0.230 & 0.210 & 0.199 & 0.176 \\
\hline 0.510 & 0.335 & 0.228 & 0.208 & 0.163 \\
\hline 0.521 & 0.335 & 0.236 & 0.215 & 0.180 \\
\hline 0,532 & .0 .335 & 0.252 & 0.225 & 0,100 \\
\hline 0.543 & 0.335 & 0.269 & 0.241 & 0.200 \\
\hline 0.556 & 0.335 & 0.283 & 0.255 & 0.220 \\
\hline 0.568 & 0.437 & 0.295 & 0.268 & 0.231 \\
\hline 0.581 & 0.437 & 0.306 & 0.276 & 0.241 \\
\hline 0.585 & 0.437 & 0.314 & 0.286 & 0.250 \\
\hline 0.610 & 0.437 & 0.322 & 0.284 & 0.321 \\
\hline 0.625 & 0.477 & 0.437 & 0.303 & 0.321 \\
\hline 0.641 & 0.677 & 0.137 & 0.310 & 0.321 \\
\hline 0.658 & 0.477 & 0,437 & 0.340 & 0.321 \\
\hline 0.676 & 0.177 & 0.437 & 0,359 & 0.321 \\
\hline 0.694 & 0.477 & 0.137 & 0.370 & 0.321 \\
\hline 0.714 & 0.177 & 0.437 & 0.372 & 0.321 \\
\hline 0.735 & 0.477 & 0.444 & 0.397 & 0.313 \\
\hline 0.962 & 0.488 & 0.444 & 0.397 & 0.343 \\
\hline 1.000 & 0,520 & 0.469 & 0.397 & 0.343 \\
\hline 1.067 & 0.520 & $0,4 \$ 5$ & 0.397 & 0.343 \\
\hline 1.233 & 0.520 & 0.458 & 0.387 & 0.343 \\
\hline 2.358 & 0.554 & 0.514 & 0.458 & 0.411 \\
\hline 2.410 & 0.554 & 0.514 & 0.458 & 0.411 \\
\hline 2,463 & 0.554 & 0.514 & $.0,458$ & 0.411 \\
\hline 2.519 & 0.554 & 0.514 & 0.458 & 0.111 \\
\hline 2.577 & 0.554 & 0.514 & 0.450 & 0.411 \\
\hline 3.460 & 0.565 & 0.517 & 0.465 & 0.614 \\
\hline 3.571 & 0,565 & 0.517 & 0.451 & 0,390 \\
\hline 5.025 & 0.576 & 0.518 & 0.451 & 0.390 \\
\hline 5.283 & 0.573 & 0.507 & 0.451 & 0.390 \\
\hline 5.525 & 0.573 & 0.507 & 0,451 & 0.390 \\
\hline 5.814 & 0.573 & 0.507 & 0.451 & 0.380 \\
\hline 5,135 & 0.573 & 0.507 & 0,451 & 0.390 \\
\hline 6.494 & 0.573 & 0.496 & 0.428 & 0.376 \\
\hline 6,897 & 0.573 & 0.486 & 0.420 & 0.376 \\
\hline 7.353 & 0.573 & 0.496 & 0.420 & 0.376 \\
\hline 7.874 & 0.443 & 0.417 & $0.12 \theta$ & 0.376 \\
\hline 8.475 & 0.400 & 0.388 & 0.350 & 0.325 \\
\hline 9.174 & 0.376 & 0.353 & 0.322 & 0.295 \\
\hline 10.000 & 0.344 & 0.317 & 0.286 & 0.260 \\
\hline 10.163 & 0.314 & 0,317 & 0.286 & 0.260 \\
\hline 10.331 & 0.344 & 0.317 & 0.286 & 0.260 \\
\hline 10,504 & 0.344 & 0.317 & 0.286 & 0.260 \\
\hline 10,684 & 0.344 & 0.317 & 0.286 & 0.260 \\
\hline 10.870 & 0.314 & 0.317 & 0.286 & 0.260 \\
\hline 11,062 & 0.344 & 0.317 & 0.286 & 0.200 \\
\hline 11.261 & 0.344 & 0.317 & 0.288 & 0.260 \\
\hline 11.468 & 0.344 & 0.317 & 0.280 & 0.260 \\
\hline 11,682 & 0,344 & 0.317 & 0.286 & 0.260 \\
\hline 14.368 & 0.336 & 0.306 & 0.272 & 0.240 \\
\hline $14.70 \mathrm{~b}$ & 0.036 & 0.306 & 0.272 & 0.240 \\
\hline 17.606 & 0.260 & 0.250 & 0.239 & 0.227 \\
\hline 18.116 & 0.260 & 0.251 & 0,239 & 0.227 \\
\hline 18.841 & 0.260 & 0.251 & 0.238 & 0.227 \\
\hline 20.492 & 0.260 & 0.251 & 0.238 & 0.227 \\
\hline 21.186 & 0.260 & 0,251 & 0.238 & 0.227 \\
\hline 21.830 & 0.260 & 0.251 & 0.238 & 0.227 \\
\hline 22.727 & 0.242 & 0.232 & 0.225 & 0.210 \\
\hline 23.585 & 0.242 & 0.232 & 0.225 & 0.210 \\
\hline 32.051 & 0.220 & 0.213 & 0.200 & 0.208 \\
\hline 33.784 & 0.220 & 0.209 & 0.208 & 0,208 \\
\hline 35.714 & 0.209 & 0,209 & 0.200 & $0.20 \mathrm{a}$ \\
\hline 37.870 & 0.209 & 0.209 & 0,208 & 0,208 \\
\hline 46.296 & 0.200 & 0.200 & 0.200 & 0.200 \\
\hline 50.000 & 0.209 & 0.209 & 0.200 & $0.20 \mathrm{~J}$ \\
\hline
\end{tabular}


FIGURE C-2

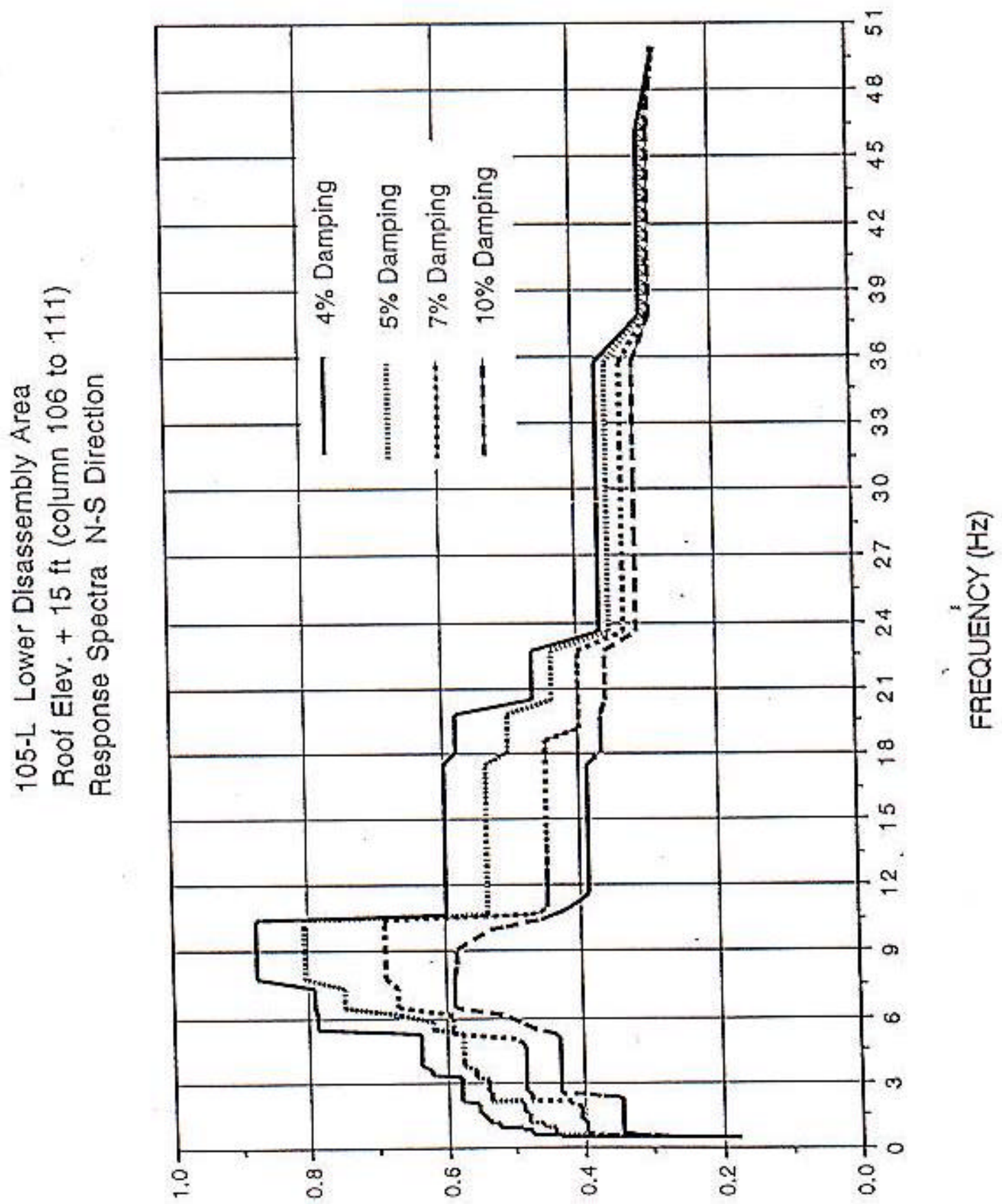

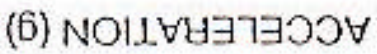


WSRC-TR-97-00289

Revision 0

Page C4 of C30

TABLE C-2

105-L LOWER DISASSEMBLY AREA (Column 106 to 111) Elev. $+15 \mathrm{ft}$ Roof RESPONSE SPECTRA

N-S DIRECTION

\begin{tabular}{|c|c|c|c|c|}
\hline $\begin{array}{l}\text { FEOUENY } \\
\text { (Hz) }\end{array}$ & 4X DAMPWA & $\begin{array}{l}\text { COELEPUTION } \\
\text { 5\% DAMPANG }\end{array}$ & TX, DAMPANG & $10 \times$ DNMPING \\
\hline 0.500 & 0.231 & 0.210 & 0.200 & 0,176 \\
\hline 0.510 & 0.338 & 0.229 & 0.208 & 0.184 \\
\hline 0.521 & 0.338 & 0.236 & 0.216 & 0.101 \\
\hline 0,532 & 0.338 & 0.252 & 0.226 & 0.196 \\
\hline 0.543 & 0.338 & 0.270 & 0.242 & 0.209 \\
\hline 0.556 & 0.338 & 0.264 & 0.255 & 0.221 \\
\hline 0.568 & 0,438 & 0.295 & 0.267 & 0.232 \\
\hline 0.581 & 0.438 & 0.308 & 0.277 & 0.242 \\
\hline 0.525 & 0.438 & 0.315 & 0.286 & 0.251 \\
\hline 0.610 & 0.438 & 0.323 & 0.295 & 0.322 \\
\hline 0.625 & 0.478 & 0.438 & 0.304 & 0,322 \\
\hline 0.641 & 0.478 & 0.438 & 0,320 & 0.322 \\
\hline 0.658 & 0.470 & 0.438 & 0.341 & 0.322 \\
\hline 0.676 & 0.478 & 0,438 & 0.360 & 0.322 \\
\hline 0.694 & 0.478 & 0,438 & 0.372 & 0.322 \\
\hline 0.714 & 0.478 & 0.438 & 0.374 & 0,322 \\
\hline 0.735 & 0.478 & 0.446 & 0.390 & 0.345 \\
\hline 0.062 & 0.491 & 0.446 & 0.399 & 0.345 \\
\hline 1,000 & 0.524 & 0.452 & 0.399 & 0.345 \\
\hline 1.067 & 0.524 & 0.458 & 0.399 & 0.345 \\
\hline 1.233 & 0.524 & 0.463 & 0.399 & 0.345 \\
\hline 2.358 & 0.576 & 0.535 & 0.476 & 0.345 \\
\hline 2.410 & 0.576 & 0,535 & 0.476 & 0.355 \\
\hline 2.463 & 0.576 & 0.535 & 0.476 & 0.375 \\
\hline 2.519 & 0.576 & 0.535 & 0.476 & 0.398 \\
\hline 2.577 & 0.576 & 0.535 & 0.476 & 0.433 \\
\hline 3.460 & 0.619 & 0.554 & 0.485 & 0.433 \\
\hline 3.571 & 0.619 & 0,554 & 0.485 & 0.433 \\
\hline 5.025 & 0.636 & 0.572 & 0.498 & 0.433 \\
\hline 5.263 & 0.636 & 0.572 & 0.567 & 0,442 \\
\hline 5.525 & 0.785 & 0.615 & 0.567 & 0.460 \\
\hline 5.814 & 0.705 & 0.615 & 0.587 . & 0.480 \\
\hline 6.135 & 0.785 & 0.664 & 0.587 & 0.515 \\
\hline 6.494 & 0.790 & 0.744 & 0.668 & 0.584 \\
\hline 6.697 & 0.790 & 0.744 & 0.668 & 0.584 \\
\hline 7.353 & 0.790 & 0.744 & 0.668 & 0.584 \\
\hline 7.874 & 0.873 & 0.803 & 0.686 & 0.584 \\
\hline 6.475 & 0.873 & 0.803 & 0.686 & 0.581 \\
\hline 9.174 & 0.873 & 0.803 & 0.686 & 0.581 \\
\hline 10.000 & 0.873 & 0.003 & 0.686 & 0.532 \\
\hline 10.163 & 0.073 & 0.803 & 0.686 & 0,509 \\
\hline 10,331 & 0.873 & 0.603 & 0.686 & 0.401 \\
\hline 10.504 & 0.873 & 0.803 & 0.686 & 0.458 \\
\hline 10.684 & 0.505 & 0.535 & 0.474 & 0.440 \\
\hline 10,070 & 0.595 & 0.535 & 0.458 & 0.420 \\
\hline 11,062 & 0.595 & 0,535 & 0.448 & 0.416 \\
\hline 11.261 & 0.585 & 0.535 & 0.448 & 0.405 \\
\hline 11.468 & 0.595 & 0.535 & 0.448 & 0.305 \\
\hline 11.682 & 0.595 & 0.535 & 0.448 & 0.388 \\
\hline 14.368 & 0.595 & 0.535 & 0.448 & 0.388 \\
\hline 14.706 & 0.595 & 0.535 & 0.448 & 0.388 \\
\hline 17.606 & 0.505 & 0.535 & 0.448 & 0.388 \\
\hline 18.116 & 0,575 & 0.503 & 0.448 & 0.366 \\
\hline 19.841 & 0.575 & 0.503 & 0,398 & 0.366 \\
\hline 20.492 & 0.466 & 0.438 & 0.390 & 0.360 \\
\hline 21.186 & 0,466 & 0.438 & 0.398 & 0.360 \\
\hline 21.030 & 0,466 & 0.438 & 0.398 & 0.380 \\
\hline 22.727 & 0.466 & 0.438 & 0.380 & 0.300 \\
\hline 23.585 & 0.366 & 0.352 & 0.331 & 0.314 \\
\hline 32.051 & 0.366 & 0.352 & 0.331 & 0.311 \\
\hline 33.704 & 0.366 & 0.352 & 0.331 & 0.311 \\
\hline 35.714 & 0.366 & 0,352 & 0.331 & 0.311 \\
\hline 37,879 & 0.300 & 0.206 & 0.201 & 0.286 \\
\hline 46.296 & 0,300 & 0.296 & 0.201 & 0.286 \\
\hline 50.000 & 0.275 & 0.276 & 0.277 & 0.278 \\
\hline
\end{tabular}


FIGURE C-3

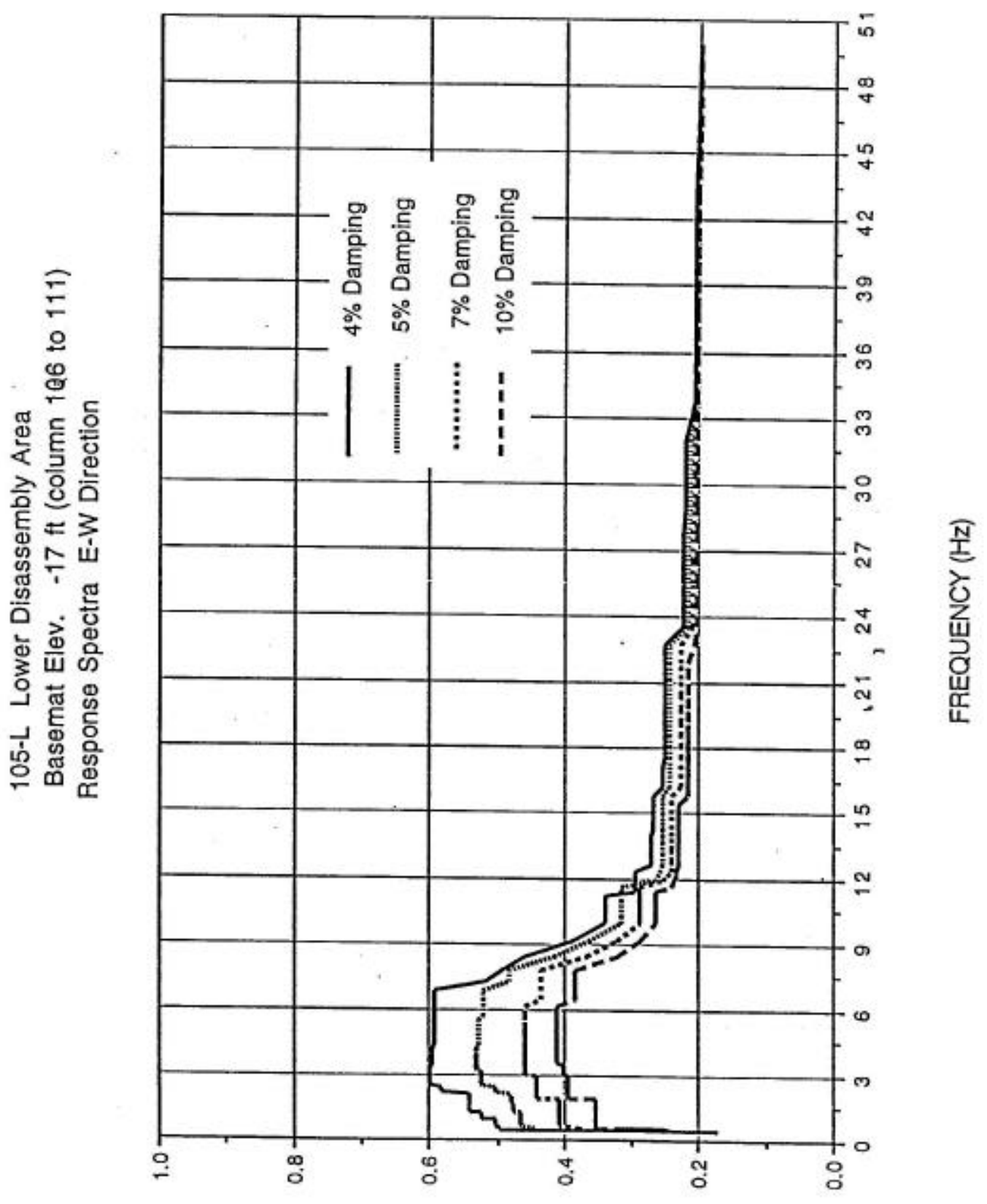

(6) NOแะษ 
WSRC-TR-97-00289

Revision 0

Page C6 of C30

\section{TABLE C-3}

105-L LOWEA DISASSEMBL.Y AREA (Column 106 to 111)

Elov. -17 it BAsemat RESPONSE SPECTAA

E-W DIRECTION

\begin{tabular}{|c|c|c|c|c|}
\hline $\begin{array}{l}\text { FABaUNCY } \\
(\mathrm{Hz})\end{array}$ & $4 \times$ DAMPUNG & $\begin{array}{l}\text { CCELERATION } \\
5 \times \text { OAMPNG }\end{array}$ & $\begin{array}{l}\text { (a) } \\
\text { Tx DAMPNo }\end{array}$ & 10\% DAMPING \\
\hline 0.500 & 0.242 & 0.221 & 0.192 & 0.172 \\
\hline 0.510 & 0.273 & 0.250 & 0.214 & 0.180 \\
\hline 0.521 & 0.296 & 0.273 & 0.234 & 0.190 \\
\hline 0.532 & 0,322 & 0.226 & 0.371 & 0.204 \\
\hline 0.543 & 0.354 & 0.321 & 0.371 & 0.220 \\
\hline 0.556 & 0.495 & 0,445 & 0.371 & 0.230 \\
\hline 0,568 & 0.495 & 0,445 & 0.371 & 0.257 \\
\hline 0,581 & 0.495 & 0.445 & 0.371 & 0.273 \\
\hline 0.595 & 0.495 & 0.445 & 0.371 & 0.286 \\
\hline 0.610 & 0.495 & 0.445 & 0.371 & 0.207 \\
\hline 0.625 & 0.495 & 0.445 & 0.371 & 0.304 \\
\hline 0.641 & 0,495 & 0.445 & 0.371 & 0.308 \\
\hline 0.658 & 0.405 & 0.445 & 0.375 & 0.353 \\
\hline 0.676 & 0.502 & 0.464 & 0.407 & 0.353 \\
\hline 0.694 & 0.502 & 0.484 & 0.407 & 0.353 \\
\hline 0.714 & 0.502 & 0,464 & 0,407 & 0,353 \\
\hline 0.735 & 0.502 & 0.464 & 0.407 & 0.353 \\
\hline 0.962 & 0.502 & 0.464 & 0,407 & 0.353 \\
\hline 1.000 & 0,502 & 0,464 & 0,407 & 0.353 \\
\hline 1.067 & 0.523 & 0.464 & 0.407 & 0.353 \\
\hline 1.233 & 0,523 & 0.464 & 0.407 & 0.353 \\
\hline 2.358 & 0.580 & 0.503 & 0.440 & 0.393 \\
\hline 2.410 & 0,580 & 0.503 & 0.440 & 0.393 \\
\hline 2.463 & 0.580 & 0.503 & 0.440 & 0.393 \\
\hline 2.518 & 0.580 & 0.503 & 0.440 & 0.393 \\
\hline 2.577 & 0.598 & 0.522 & 0.440 & 0.303 \\
\hline 3.480 & 0.595 & 0.530 & 0.457 & 0.398 \\
\hline 3.571 & 0.595 & 0,530 & 0.458 & 0.409 \\
\hline 5.025 & 0.590 & 0.527 & 0,450 & 0.411 \\
\hline 5.263 & 0,590 & 0.527 & 0.450 & 0.411 \\
\hline 5.525 & 0.500 & 0.527 & 0.459 & 0.411 \\
\hline 5.014 & 0.590 & 0.520 & 0.450 & 0.411 \\
\hline 6.135 & 0.590 & 0.520 & 0.459 & 0.411 \\
\hline 6.404 & 0.590 & 0.520 & 0.432 & 0.383 \\
\hline 6.897 & 0.590 & 0.520 & 0.432 & 0.983 \\
\hline 7.353 & 0.514 & 0.482 & 0.432 & 0.383 \\
\hline 7.874 & 0.402 & 0.402 & 0,432 & 0,383 \\
\hline 8.475 & 0.457 & 0.415 & 0.364 & 0.322 \\
\hline 0.174 & 0.387 & 0.361 & 0.320 & 0.285 \\
\hline 10.000 & 0.337 & 0.314 & 0.286 & 0.262 \\
\hline 10.163 & 0.397 & 0.314 & 0.286 & 0.262 \\
\hline 10,331 & 0,337 & 0.314 & 0.286 & 0.262 \\
\hline 10.504 & 0.337 & 0.314 & 0.286 & 0.262 \\
\hline 10,684 & 0.337 & 0.314 & 0.286 & 0.262 \\
\hline 10.870 & 0.337 & 0.314 & 0.286 & 0.262 \\
\hline 11.062 & 0.337 & 0.314 & 0.200 & 0.262 \\
\hline 11.261 & 0.337 & 0.314 & 0.286 & 0.262 \\
\hline 11,468 & 0.203 & 0.314 & 0.280 & 0,262 \\
\hline 11.682 & 0.203 & , 0.314 & 0.286 & 0.230 \\
\hline 14.368 & 0.267 & 0.254 & 0.230 & 0.220 \\
\hline 14.706 & 0.267 & 0.254 & 0.230 & $0.22 \theta$ \\
\hline 17.600 & 0.250 & 0.241 & 0.227 & 0.215 \\
\hline 18.116 & 0.250 & 0.241 & 0.227 & 0.215 \\
\hline 10.841 & 0.250 & 0.241 & 0.227 & 0.215 \\
\hline 20.492 & 0.250 & 0.241 & 0.227 & 0.215 \\
\hline 21.186 & 0,250 & 0.241 & 0.227 & 0.215 \\
\hline 21.930 & 0.250 & 0.241 & 0.227 & 0.215 \\
\hline 22.727 & 0.250 & 0.241 & 0.227 & 0.206 \\
\hline $23.5 a s$ & 0.223 & 0.216 & 0.207 & 0.202 \\
\hline 32.051 & 0.219 & 0.213 & 0.206 & 0.202 \\
\hline 33.704 & 0.206 & 0.205 & 0.206 & 0.202 \\
\hline 35.714 & 0.206 & 0.205 & 0.203 & 0.201 \\
\hline 37.679 & 0.206 & 0.205 & 0,203 & 0.201 \\
\hline 46,296 & 0.202 & 0.201 & 0.201 & 0,199 \\
\hline 50.000 & 0.190 & 0.198 & 0.198 & 0.180 \\
\hline
\end{tabular}


FIGURE C-4

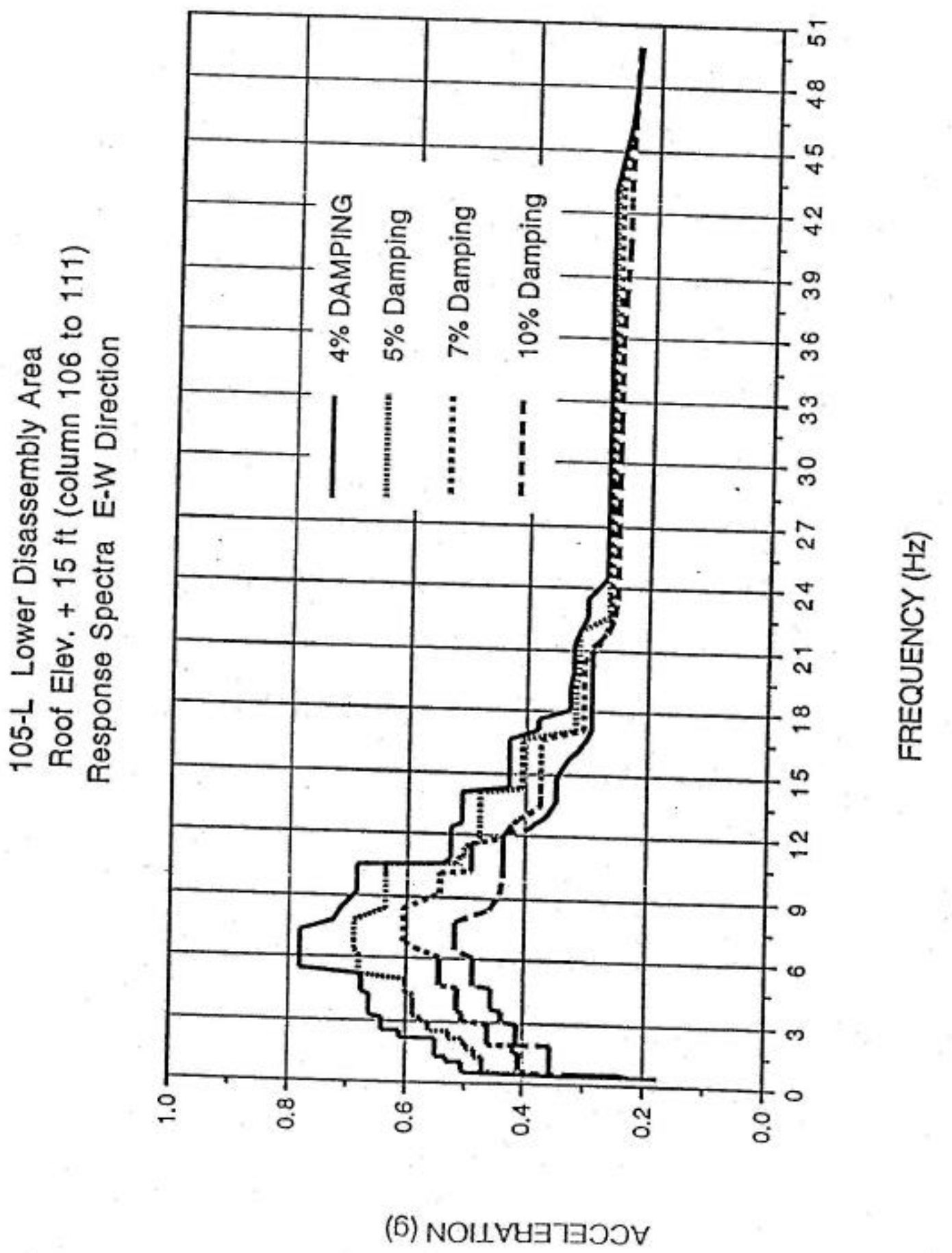


WSRC-TR-97-00289

Revision 0

Page C8 of C30

\section{TABLE C-4}

105-L. LOWER DISASSEMBLY AAEA (Colum 106 to 111) Elav. + 15 th Poof RESPONSE SPECTRA

E-W DIRECTION

\begin{tabular}{|c|c|c|c|c|}
\hline $\begin{array}{l}\text { Freolentr } \\
(\mathrm{Hz})\end{array}$ & 1\% DAMPENG & $\begin{array}{l}\text { CCELERATKON } \\
\text { 5\%, DAMPINa }\end{array}$ & $\begin{array}{l}\text { (d) } \\
7 \% \text { DAMPMA }\end{array}$ & $10 \%$ DNMPING \\
\hline 0,500 & 0.242 & 0.222 & 0,192 & 0.173 \\
\hline 0.510 & 0.274 & 0.251 & 0.214 & 0.181 \\
\hline 0.521 & 0.297 & 0.274 & 0.234 & 0.101 \\
\hline 0.532 & 0.322 & 0.207 & 0.372 & 0.205 \\
\hline 0.543 & 0.355 & 0.322 & 0.372 & 0.221 \\
\hline 0.556 & 0.496 & 0.446 & 0.372 & 0.240 \\
\hline 0.568 & 0.196 & 0.146 & 0,372 & 0.257 \\
\hline 0.501 & 0,496 & 0,446 & 0.372 & 0.273 \\
\hline 0.595 & 0.496 & 0.446 & 0.372 & 0.287 \\
\hline 0.610 & 0,496 & 0.446 & 0.372 & 0.288 \\
\hline 0,625 & 0.496 & 0.446 & 0.372 & 0.306 \\
\hline 0.641 & 0.496 & 0.446 & 0.372 & 0.309 \\
\hline 0.658 & 0.496 & 0.440 & 0.376 & 0.355 \\
\hline 0.676 & 0.504 & 0.466 & 0.408 & 0.355 \\
\hline 0.694 & 0,504 & 0.468 & 0.408 & 0.355 \\
\hline 0.714 & 0.504 & 0.466 & 0.408 & 0.355 \\
\hline 0.735 & 0.504 & 0.466 & 0.408 & 0.955 \\
\hline 0.062 & 0.504 & 0.466 & 0.400 & 0.355 \\
\hline 1.000 & 0.504 & 0.468 & 0.400 & 0.355 \\
\hline 1.067 & 0.527 & 0.466 & 0.400 & 0.355 \\
\hline 1.233 & 0.527 & 0.466 & 0,408 & 0.355 \\
\hline 2.350 & 0.600 & 0.526 & 0.460 & 0.412 \\
\hline 2.410 & 0.608 & 0.526 & 0.460 & 0.412 \\
\hline 2.463 & 0.608 & 0.526 & 0.460 & 0.412 \\
\hline 2.510 & 0.608 & 0.526 & 0.160 & 0.412 \\
\hline 2.577 & 0.639 & 0.558 & 0.460 & 0.412 \\
\hline 3.460 & 0.650 & 0.586 & 0.503 & 0.438 \\
\hline 3.571 & 0.658 . & 0.586 & 0.500 & 0.438 \\
\hline 5.025 & 0.674 & 0.600 & 0.543 & 0.484 \\
\hline 5.263 & 0.677 & 0.677 & 0.543 & 0.484 \\
\hline 5.525 & 0.777 & 0.677 & 0.543 & 0.484 \\
\hline 5.014 & 0.777 & 0.677 & 0.543 & 0.481 \\
\hline 6.135 & 0.777 & 0.677 & 0.543 & 0.484 \\
\hline 6.494 & 0.777 & 0,687 & 0.576 & 0.516 \\
\hline 6.897 & 0.777 & 0.687 & 0.603 & 0.516 \\
\hline 7.353 & 0.777 & 0.687 & 0.603 & 0.516 \\
\hline 7.074 & 0.720 & 0.687 & 0.603 & 0.516 \\
\hline 8.475 & 0.705 & 0.633 & 0.603 & 0.459 \\
\hline 9.174 & 0.682 . & 0.833 & 0.5 .43 & 0.442 \\
\hline 10,000 & $0.683^{\circ}$ & 0.633 & 0.543 & 0.436 \\
\hline 10.163 & 0.683 & 0.633 & 0.543 & 0.436 \\
\hline 10,331 & 0.683 & 0.633 & 0.488 & 0.436 \\
\hline 10.504 & 0.683 & 0.633 & 0.489 & 0.436 \\
\hline 10.684 & 0,538 & 0.517 & 0,480 & 0.436 \\
\hline 10,870 & 0.526 & 0.510 & 0,489 & 0,436 \\
\hline 11.062 & 0.526 & 0.503 & 0.489 & 0.436 \\
\hline 11.261 & 0.526 & 0.502 & 0.489 & 0,436 \\
\hline 11.468 & 0.526 & 0.500 & 0.480 & 0.436 \\
\hline 11.682 & 0,526 & 0.460 & 0.480 & 0.430 \\
\hline 14,368 & 0.429 & 0,406 & 0.375 & 0.350 \\
\hline 14.700 & 0.420 & 0.106 & 0.375 & 0.349 \\
\hline 17.606 & 0.380 & 0.317 & 0.205 & 0.201 \\
\hline 18.116 & 0.326 & 0.217 & 0.305 & 0.201 \\
\hline 19.041 & 0.323 & 0.316 & 0.305 & 0.291 \\
\hline 20.492 & 0.323 & 0.316 & 0.305 & 0.291 \\
\hline 21.166 & 0.323 & 0.316 & 0.284 & 0.291 \\
\hline 21.930 & 0,316 & 0.316 & 0.272 & 0.267 \\
\hline 22.727 & 0.302 & 0.268 & 0.260 & 0.258 \\
\hline 23.505 & 0.302 & 0.268 & 0.260 & 0.253 \\
\hline 32.051 & 0.272 & 0,268 & 0,260 & 0.253 \\
\hline 33.784 & 0.272 & 0.268 & 0.260 & 0.253 \\
\hline 35.714 & 0.272 & 0.268 & 0.260 & 0.253 \\
\hline 37.879 & 0.271 & 0.266 & 0.258 & 0.249 \\
\hline 46.296 & 0.245 & 0.243 & 0,242 & 0.240 \\
\hline 50,000 & 0.232 & 0.232 & 0.234 & 0.235 \\
\hline
\end{tabular}


FIGURE C-5

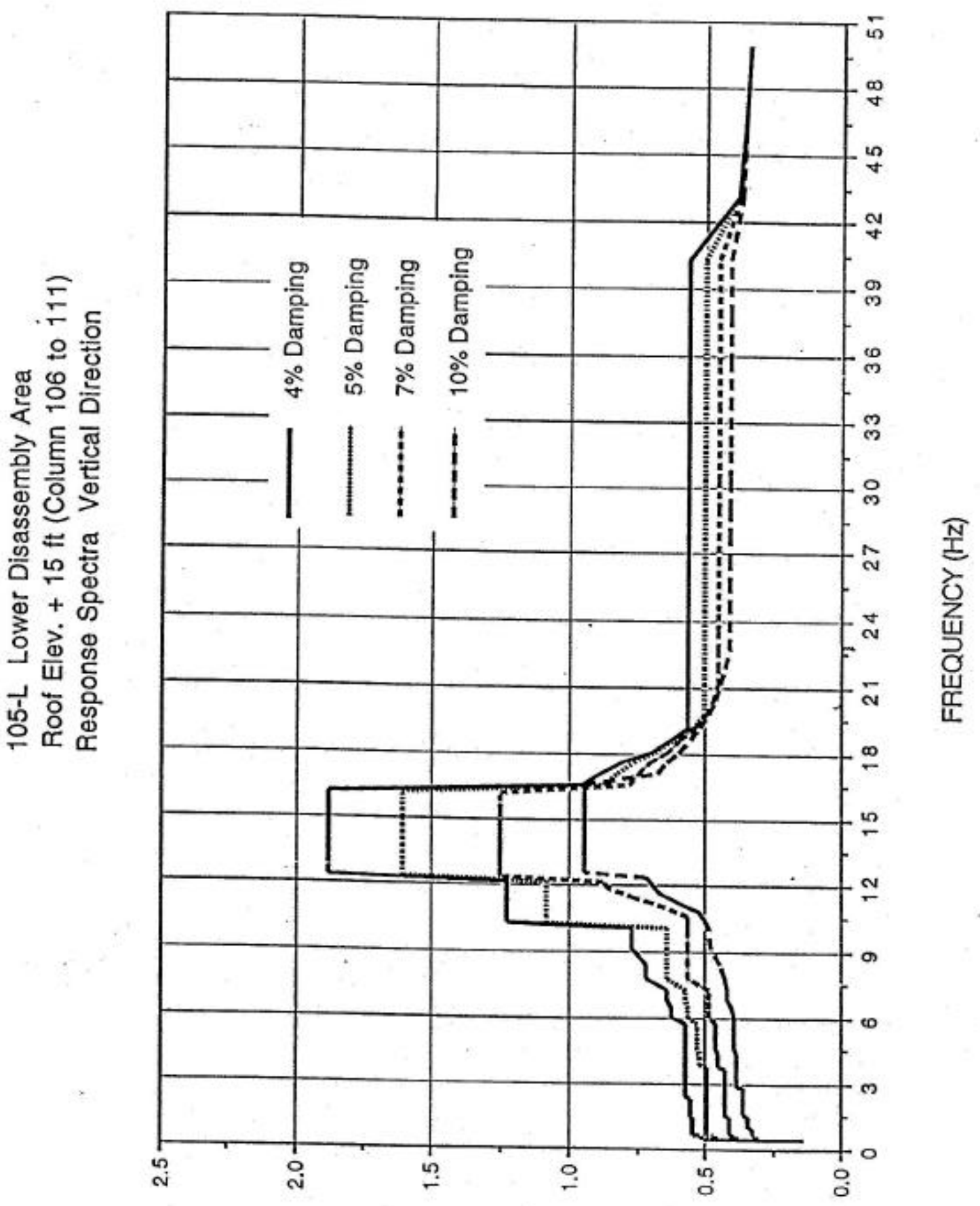

(6) NOI1 ชบำ $300 \mathrm{~V}$ 
WSRC-TR-97-00289

Revision 0

Page C10 of C30

\section{TABLE C-5}

105-L LOWER DLSASSEMBLY AREA (Colum 106 to 111) Elev. +15 tt Aloof AESPONSE SPECTRA VERTCN DERECTION

\begin{tabular}{|c|c|c|c|c|}
\hline $\begin{array}{l}\text { Frecuener } \\
{\left[\mathrm{H}_{2}\right)}\end{array}$ & 4s OAMPano & $\begin{array}{l}\text { COELERATION } \\
5 \% \text { DNANGI }\end{array}$ & 7\% DANPNa & 10\% DAMPGKG \\
\hline 0.500 & 0.100 & 0,170 & 0,159 & 0.137 \\
\hline 0.510 & 0.218 & 0.200 & 0.171 & 0.147 \\
\hline 0.521 & 0,409 & 0.450 & 0,374 & 0.161 \\
\hline 0,568 & 0.402 & 0.450 & 0.374 & 0.300 \\
\hline 0.581 & 0.400 & 0.450 & 0.374 & 0.300 \\
\hline 0.505 & 0.520 & 0.472 & 0.396 & 0.310 \\
\hline 0.610 & 0.520 & 0.472 & 0.596 & 0.310 \\
\hline 0.604 & 0.520 & 0.472 & 0.396 & 0.310 \\
\hline 0.750 & 0.540 & 0.482 & 0,407 & 0.325 \\
\hline 0,928 & 0.549 & 0.402 & 0.407 & 0.325 \\
\hline 0,962 & 0.549 & 0.482 & 0.407 & 0.325 \\
\hline 1.000 & 0.540 & 0.492 & 0,407 & 0.325 \\
\hline 1.070 & 0.549 & 0.492 & 0,407 & 0,341 \\
\hline$t .088$ & 0.548 & 0.402 & 0.407 & 0.344 \\
\hline 1.133 & 0,549 & 0.402 & 0.407 & 0.344 \\
\hline 1,146 & 0.540 & 0.492 & 0,407 & 0.344 \\
\hline 1.156 & 0.549 & 0,492 & 0,407 & 0,314 \\
\hline 1.168 & 0.549 & 0.492 & 0.407 & 0.344 \\
\hline 1.520 & 0.549 & 0.492 & 0.407 & 0.344 \\
\hline $1.54 t$ & 0.557 & 0.495 & 0.407 & 0.344 \\
\hline 1.246 & 0,557 & 0.405 & 0.423 & 0.350 \\
\hline 1.980 & 0.557 & 0.485 & 0.423 & 0.350 \\
\hline 2.262 & 0.557 & 0.485 & 0.423 & 0.350 \\
\hline 2.309 & 0.557 & 0.465 & 0.423 & 0.350 \\
\hline 2.350 & 0.557 & 0.405 & 0.423 & 0.359 \\
\hline 2.410 & 0.557 & 0.485 & 0,423 & 0.359 \\
\hline 2.463 & 0.572 & 0.408 & 0.423 & 0,359 \\
\hline 2.518 & 0.572 & 0.408 & 0.423 & 0.350 \\
\hline 3,077 & 0.572 & 0.498 & 0.431 & 0.381 \\
\hline 3.165 & 0.572 & 0,408 & 0,431 & 0.361 \\
\hline 3.571 & 0.572 & 0.408 & 0.431 & 0.381 \\
\hline 3.690 & 0.572 & 0.496 & 0.431 & 0.301 \\
\hline 3.817 & 0.572 & 0.486 & 0.452 & 0.351 \\
\hline 3.953 & 0.572 & 0.517 & 0,452 & 0.385 \\
\hline 4,425 & 0,576 & 0.517 & 0.452 & 0.385 \\
\hline 4,605 & 0.576 & 0.532 & 0.463 & 0.390 \\
\hline 4.005 & 0.576 & 0,532 & 0,483 & 0.390 \\
\hline 5.614 & 0.576 & 0,532 & 0.403 & 0,390 \\
\hline 6.135 & 0.623 & 0.504 & 0.409 & 0.390 \\
\hline 6.494 & 0.623 & 0,564 & 0.488 & 0,402 \\
\hline 6.607 & $0.64 t$ & 0,575 & 0.488 & 0.421 \\
\hline 7.353 & 0.641 & 0.575 & 0.489 & 0,422 \\
\hline 7.674 & 0.715 & 0,641 & 0.550 & 0,426 \\
\hline 8.475 & 0.715 & 0.644 & 0.550 & 0.444 \\
\hline 0.174 & 0.764 & 0.644 & 0.558 & 0.476 \\
\hline 10.000 & 0.764 & 0,644 & 0.559 & 0.466 \\
\hline 10.331 & 1.221 & 1.073 & 0.559 & 0.482 \\
\hline 10,504 & 1.221 & 1.073 & 0.550 & 0.500 \\
\hline 10.684 & 1.221 & 1.073 & 0.584 & 0.509 \\
\hline 10.570 & 1.221 & 1.073 & 0.600 & 0,524 \\
\hline 11.062 & 1.221 & 1.073 & 0.651 & 0.562 \\
\hline 11.261 & 1.221 & 1.073 & 0.702 & 0.500 \\
\hline 11.468 & 1.221 & 1.073 & 0.756 & 0,620 \\
\hline 11.602 & 1.221 & 1.073 & 0.004 & 0,664 \\
\hline 11.905 & 1.221 & 1.073 & 0.853 & 0.605 \\
\hline 12,136 & 1.221 & 1.073 & 0.000 & 0.702 \\
\hline 12.376 & 1.075 & 1.005 & 1.245 & 0.714 \\
\hline 12.826 & 1.675 & 1.tes & 1.245 & 0.030 \\
\hline 13.736 & 1.675 & 1.605 & 1.245 & 0,039 \\
\hline 14.045 & 1.075 & 1.605 & 1.245 & 0.830 \\
\hline 14.368 & 1.675 & $1 .=25$ & 1.245 & 0.039 \\
\hline 14.706 & 1.675 & 1.605 & 1.245 & 0,039 \\
\hline 33.784 & 0.567 & 0.504 & 0.456 & 0.412 \\
\hline 35.714 & 0.567 & 0.504 & 0.456 & 0.412 \\
\hline 37,879 & 0.567 & 0,504 & 0,456 & 0.412 \\
\hline 40,323 & 0.567 & 0.504 & 0.456 & 0,112 \\
\hline 43.103 & 0.362 & 0.300 & 0.376 & 0.371 \\
\hline 46.296 & 0.356 & 0,356 & 0.356 & 0.355 \\
\hline 50.000 & 0.330 & 0.339 & 0.341 & 0.343 \\
\hline
\end{tabular}


FIGURE C-6

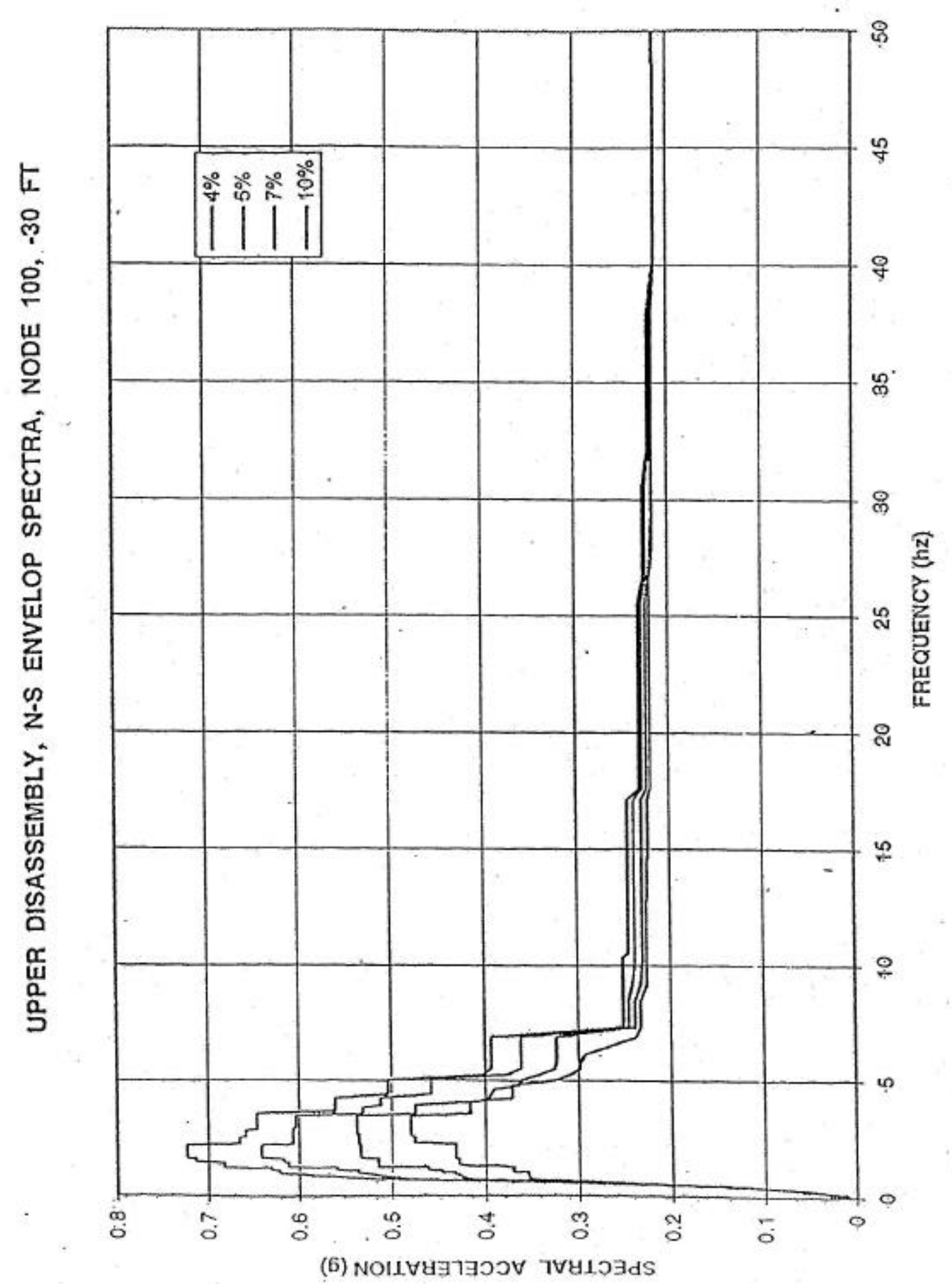




\section{TABLE C-6}

Upper Disassembly, cavelop spectra, N-S , node 250 , EL -30 , broadened $15 \%$

\begin{tabular}{|c|c|c|c|c|c|c|c|c|c|c|c|c|c|c|}
\hline Frequenc & $4 \%$ & $5 \%$ & $7 \%$ & $10 \%$ & Frequency & $4 \%$ & $5 \%$ & $7 \%$ & $10 \%$ & Frequency & $4 \%$ & $5 \%$ & $7 \%$ & $10 \%$ \\
\hline & & .010 & .0102 & .0099 & 1.479 & .7142 & .6121 & .5145 & .4282 & 8.475 & .2519 & .2461 & .2390 & .2334 \\
\hline $\begin{array}{l}.100 \\
.104\end{array}$ & $\begin{array}{l}.0104 \\
.0104\end{array}$ & .0103 & .0102 & .0100 & 1.499 & .7142 & .6176 & .5145 & .4282 & 9.174 & .2519 & .2418 & .2326 & .2263 \\
\hline $\begin{array}{l}.104 \\
.108\end{array}$ & $\begin{array}{l}.0104 \\
.0112\end{array}$ & .0111 & .0110 & .0109 & 1.520 & .7142 & .6176 & .5145 & .4282 & 10.000 & .2519 & .2392 & .2310 & .2261 \\
\hline .108 & $\begin{array}{l}.0112 \\
.0123\end{array}$ & .0122 & .0119 & .0117 & 1.541 & .7142 & .6176 & .5145 & .4282 & 10.000 & .2519 & .2392 & .2310 & .2261 \\
\hline .112 & $\begin{array}{l}.0123 \\
.0134\end{array}$ & .0132 & .0129 & .0124 & 1.562 & .7142 & .6176 & 5145 & 4282 & 10.163 & 2519 & .2392 & .2309 & .2261 \\
\hline .117 & $\begin{array}{l}.0134 \\
.0157\end{array}$ & .0153 & .0148 & .0141 & 1.585 & .7142 & .6176 & .5145 & 4282 & 10.331 & .2519 & .2392 & .2309 & .2261 \\
\hline .122 & $\begin{array}{l}.0157 \\
.0296\end{array}$ & $\begin{array}{r}.0153 \\
.0277\end{array}$ & .0247 &, 0164 & 1.608 & .7142 & .6176 & .5145 & .4282 & 10.504 & .2455 & .2392 & .2309 & .2261 \\
\hline .128 & $\begin{array}{l}.0296 \\
.0296\end{array}$ & $\begin{array}{l}.0277 \\
.0277\end{array}$ & .0247 & .0189 & 1.631 & .7142 & .6176 & .5145 & .4318 & 10.684 & .2455 & .2392 & .2309 & .2261 \\
\hline .134 & $\begin{array}{l}.0296 \\
.0296\end{array}$ & $\begin{array}{l}.0277 \\
.0277\end{array}$ & .0247 & .0208 & 1.656 & .7235 & .6421 & .5340 & .4318 & 10.870 & .2455 & .2392 & .2309 & .2261 \\
\hline .140 & $\begin{array}{l}.0296 \\
.0296\end{array}$ & $\begin{array}{l}.0277 \\
.0277\end{array}$ & .0247 & .0215 & 1.681 & .7235 & .6421 & .5340 & .4318 & 11.062 & .2455 & .2392 & .2309 & .2261 \\
\hline .148 & $\begin{array}{l}.0296 \\
.0296\end{array}$ & $\begin{array}{l}.0277 \\
.0277\end{array}$ & .0247 & .0216 & 1.706 & .7235 & .6421 & .5340 & .4318 & 11.261 & .2455 & .2392 & .2309 & .2261 \\
\hline .156 & $\begin{array}{l}.0296 \\
.0297\end{array}$ & $\begin{array}{l}.0277 \\
.0285\end{array}$ & .0265 & .0241 & 1.733 & .7235 & .6421 & .5340 & .4318 & 11.468 & .2455 & .2392 & .2309 & .2261 \\
\hline .166 & $\begin{array}{l}.0297 \\
.0346\end{array}$ & $\begin{array}{l}.0285 \\
.0331\end{array}$ & .0305 & .0273 & 1.761 & .7235 & .6421 & .5340 & .4318 & 11.682 & .2455 & .2392 & .2309 & .2261 \\
\hline .176 & $\begin{array}{l}.0346 \\
.0358\end{array}$ & $\begin{array}{l}.0331 \\
.0343\end{array}$ & .0316 & .0296 & 1.789 & .7235 & .6421 & .5340 & .4318 & 11.905 & .2455 & .2392 & .2309 & .2261 \\
\hline .188 & $\begin{array}{l}.0358 \\
.0595\end{array}$ & $\begin{array}{l}.0343 \\
.0552\end{array}$ & .0485 & .0326 & 1.818 & .7235 & .6421 & 5340 & .4318 & 12.136 & .2455 & .2392 & .2309 & .2261 \\
\hline .202 & $\begin{array}{l}.0595 \\
.0595\end{array}$ & $\begin{array}{l}.0552 \\
.0552\end{array}$ & .0485 & .0383 & 1.848 & .7235 & .6421 & .5340 & .4318 & 12.376 & .2455 & .2392 & .2309 & .2261 \\
\hline .217 & $\begin{array}{l}.0595 \\
.0595\end{array}$ & $\begin{array}{l}.0552 \\
.0552\end{array}$ & .0485 & .0417 & 1.880 & .7235 & .6421 & .5340 & .4318 & 12.626 & .2455 & .2392 & .2309 & .2261 \\
\hline .236 & $\begin{array}{l}.0595 \\
=0595\end{array}$ & $\begin{array}{l}.0552 \\
.0552\end{array}$ & .0485 & .0459 & 1.912 & .7235 & .6421 & .5340 & .4318 & 12.887 & .2455 & .2392 & .2309 & .2249 \\
\hline .258 & $\begin{array}{l}.0595 \\
.0595\end{array}$ & $\begin{array}{l}.0552 \\
.0552\end{array}$ & .0512 & .0504 & 1.946 & .7235 & .6421 & .5340 & .4318 & 13.158 & .2455 & .2392 & .2309 & .2249 \\
\hline .284 & $\begin{array}{l}.0595 \\
.0846\end{array}$ & $\begin{array}{l}.0552 \\
.0783\end{array}$ & .0685 & .0588 & 1.980 & .7235 & .6421 & .5340 & .4318 & 13.441 & .2455 & .2392 & .2309 & .2249 \\
\hline .316 & $\begin{array}{l}.0846 \\
.0881\end{array}$ & $\begin{array}{l}.0783 \\
.0830\end{array}$ & .0750 & .0720 & 2,016 & .7235 & .6421 & .5340 & .4318 & 13.736 & .2455 & .2392 & .2309 & .2249 \\
\hline 357 & $\begin{array}{l}.0881 \\
.1189\end{array}$ & $\begin{array}{l}.0830 \\
.1122\end{array}$ & .1044 & .0978 & 2.053 & .7235 & .6421 & .5340 & .4318 & 14.045 & .2455 & .2392 & .2309 & .2249 \\
\hline .410 & $\begin{array}{l}.1189 \\
.2175\end{array}$ & $\begin{array}{r}.1122 \\
.2068\end{array}$ & .1884 & .1673 & 2.092 & .7235 & .6421 & .5340 & .4318 & 14.368 & .2455 & .2392 & .2309 & .2249 \\
\hline .481 & $\begin{array}{l}.2175 \\
.3546\end{array}$ & $\begin{array}{l}.2068 \\
.3243\end{array}$ & 2945 & .2578 & 2,132 & .7235 & .6421 & .5340 & .4318 & 14.706 & .2455 & .2392 & .2309 & .2239 \\
\hline .581 & $\begin{array}{l}.3546 \\
.5242\end{array}$ & $\begin{array}{l}.3243 \\
.4794\end{array}$ & .4184 & 3532 & 2.174 & .7235 & .6421 & .5340 & .4318 & 15.060 & .2455 & .2392 & $: 2309$ & .2239 \\
\hline .735 & $\begin{array}{l}.5242 \\
.6215\end{array}$ & $\begin{array}{l}.4794 \\
.5370\end{array}$ & .4343 & .3553 & 2.217 & .7235 & .6421 & .5340 & .4318 & 15,432 & .2455 & .2392 & .2309 & .2239 \\
\hline 1.000 & $\begin{array}{l}.6215 \\
.6215\end{array}$ & $\begin{array}{l}.5370 \\
.5370\end{array}$ & .4343 & 3553 & 2,262 & .6658 & .6071 & .5357 & .4318 & 15.823 & .2455 & .2392 & .2309 & .2239 \\
\hline 1.000 & $\begin{array}{l}.6215 \\
.6215\end{array}$ & $\begin{array}{l}.5370 \\
.5370\end{array}$ & .4343 & .3553 & 2309 & .6658 & .6071 & .5357 & .4760 & 16.234 & .2455 & .2392 & .2309 & .2239 \\
\hline 1.009 & $\begin{array}{l}.6215 \\
.6215\end{array}$ & $\begin{array}{l}.5370 \\
.5370\end{array}$ & .4343 & .3553 & 2358 & .6658 & .6071 & .5357 & .4760 & 16.667 & .2455 & .2392 & .2309 & .2239 \\
\hline 1.018 & $\begin{array}{l}.6215 \\
.6215\end{array}$ & $\begin{array}{l}.5370 \\
.5370\end{array}$ & .4470 & 3709 & 2.410 & .6658 & .6071 & .5357 & 4760 & 17.123 & .2455 & $.239 \pi^{2}$ & .2309 & .2239 \\
\hline 1.028 & $\begin{array}{l}.6215 \\
.6215\end{array}$ & $\begin{array}{l}.5370 \\
.5370\end{array}$ & .4470 & 3709 & 2.463 & .6658 & .6071 & .5357 & 4760 & 17.606 & .2328 & .2294 & .2246 & .2206 \\
\hline 1.037 & $\begin{array}{l}.6215 \\
.6215\end{array}$ & $\begin{array}{l}.5370 \\
.5370\end{array}$ &, 4470 & 3709 & 2.519 & .6658 & .6071 & 5357 & .4760 & 18,116 & .2328 & .2294 & .2246 & .2206 \\
\hline 1.047 & $\begin{array}{l}.6215 \\
.6215\end{array}$ & $\begin{array}{l}.5370 \\
.5370\end{array}$ & .4470 & .3709 & 2.577 & .6590 & .6071 & .5357 & .4760 & 18.657 & .2328 & .2294 & .2246 & .2206 \\
\hline 1.057 & $\begin{array}{l}.6215 \\
.6215\end{array}$ & $\begin{array}{l}.5370 \\
.5370\end{array}$ & .4470 & 3709 & 2.639 & .6590 & .6071 & .5357 & .4799 & 19.231 & .2328 & .2294 & .2246 & .2206 \\
\hline 1.067 & $\begin{array}{l}.6215 \\
.6215\end{array}$ & $\begin{array}{l}.5370 \\
.5370\end{array}$ & .4470 & 3709 & 2.703 & .6590 & .6071 & .5376 & .4799 & 19.841 & .2328 & .2294 & .2246 & .2206 \\
\hline 1.078 & $\begin{array}{l}.6215 \\
.6215\end{array}$ & $\begin{array}{l}.5370 \\
5370\end{array}$ & .4470 & .3709 & 2.770 & .6590 & .6071 & .5376 & .4799 & 20.492 & .2328 & .2294 & .2246 & .2206 \\
\hline 1.088 & $\begin{array}{l}.6215 \\
.6215\end{array}$ & $\begin{array}{l}.5370 \\
.5370\end{array}$ & .4470 & .3709 & 2.841 & .6590 & .6071 & .5376 & .4799 & 21.186 & .2328 & .2294 & .2246 & .2206 \\
\hline 1.099 & $\begin{array}{l}.6215 \\
.6215\end{array}$ & $\begin{array}{l}.5370 \\
.5370\end{array}$ & .4470 & .3709 & 2.915 & .6463 & .6071 & .5376 & .4799 & 21.930 & .2328 & .2294 & .2246 & .2206 \\
\hline 1.110 & $\begin{array}{l}.6215 \\
.6307\end{array}$ & $\begin{array}{l}.5370 \\
.5602\end{array}$ & .4618 & .3709 & 2.994 & .6463 & .6037 & .5376 & .4799 & 22.727 & .2328 & .2294 & .2246 & .2206 \\
\hline 1.121 & .6307 & $\begin{array}{l}5602 \\
.5602\end{array}$ & .4618 & 3709 & 3.077 & .6463 & .6037 & .5376 & .4799 & 23.585 & .2328 & .2294 & .2246 & .2206 \\
\hline 1.133 & .6307 & $\begin{array}{l}.5602 \\
.5602\end{array}$ & .4618 & 3709 & 3.165 & .6463 & .6037 & 5376 & .4799 & 24.510 & $.2328^{\circ}$ & .2294 & .2246 & .2206 \\
\hline L.144 & .6307 & $\begin{array}{l}.5602 \\
.5602\end{array}$ & .4618 & .3709 & 3.257 & .6463 & .6037 & .5376 & .4799 & 25.510 & .2328 & .2294 & .2246 & .2206 \\
\hline 1.156 & .6307 & $\begin{array}{l}.5602 \\
.5602\end{array}$ & .4618 & .3709 & 3.356 & .6463 & .6037 & .5376 & .4799 & 26.596 & .2273 & .2247 & .2213 & .2206 \\
\hline 1.168 & .6307 & $\begin{array}{l}.5602 \\
.5602\end{array}$ & .4618 & 3709 & 3.460 & .6463 & .6037 & .5376 & .4799 & 27.778 & .2273 & .2247 & .2190 & .2166 \\
\hline 1.181 & .6307 & $\begin{array}{l}.5602 \\
5602\end{array}$ & .4618 & 3709 & 3.571 & .6463 & .5317 & .4749 & .4165 & 29.070 & .2273 & .2247 & .2180 & .2166 \\
\hline 1.193 & .6307 & $\begin{array}{l}5602 \\
.5602\end{array}$ & .4618 & 3709 & 690 & 5610 & .5317 & .4749 & .4165 & 30,488 & .2273 & .2247 & .2180 & .2166 \\
\hline 1.206 & .6307 & $\begin{array}{l}.5602 \\
.5602\end{array}$ & .4618 & .3709 & 3.817 & .5610 & .5317 & .4749 & .4165 & 32.051 & .2216 & .2200 & .2180 & .2166 \\
\hline 1.220 & .6307 & $\begin{array}{l}.5602 \\
.5602\end{array}$ & .4618 & .3709 & 3.953 & .5610 & .5120 & .4749 & .4165 & 33.784 & .2216 & .2200 & .2180 & .2166 \\
\hline 1.233 & .6831 & $\begin{array}{l}.5602 \\
.6121\end{array}$ & .4618 & .3709 & 4.098 & .5610 & .5120 & .4077 & .4165 & 35.714 & .2216 & .2200 & .2180 & .2166 \\
\hline 1.247 & .6831 & $\begin{array}{l}.6121 \\
.6121\end{array}$ & 5145 & 3726 & .255 & .5610 & .5120 & .3960 & 3717 & 37.879 & .2216 & .2200 & .2180 & .2166 \\
\hline 1.261 & .6831 & $\begin{array}{l}.6121 \\
.6121\end{array}$ & .5145 & 3804 & 4.425 & .5037 & .4581 & 3939 & 3717 & 40.323 & .2148 & .2144 & .2141 & .2140 \\
\hline 1.276 & .6831 & $\begin{array}{l}.6121 \\
.6121\end{array}$ & .5145 & .3868 & 4.608 & .5037 & -4581 & 3904 & .3717 & 43.103 & .2148 & .2144 & .2141 & .2140 \\
\hline 1.290 & .6831 & $\begin{array}{l}.6121 \\
.6121\end{array}$ & .5145 & .3926 & 4.808 & .5037 & .4581 & .3639 & .3717 & 46.296 & .2148 & .2144 & .2141 & .2140 \\
\hline 1.305 & .6831 & $\begin{array}{l}.6121 \\
.6121\end{array}$ & 5145 & .4282 & 5.025 & .5037 & .4581 & .3610 & 3249 & 50.000 & .2154 & .2153 & .2152 & .2149 \\
\hline 1.321 & .6831 & $\begin{array}{l}.6121 \\
.6121\end{array}$ & .5145 & .4282 & 5.263 & .4019 & 3777 & .3425 & 3098 & & & & & \\
\hline 1.337 & .6831 & $\begin{array}{l}.6121 \\
.6121\end{array}$ & .5145 & .4282 & 5,525 & .3929 & .3616 & 3255 & .2986 & & & & & \\
\hline 1.353 & .6831 & $\begin{array}{l}.6121 \\
.6121\end{array}$ & .5145 & .4282 & 5.814 & .3929 & .3616 & 3232 & .2982 & & & & & \\
\hline $\mathrm{t} .370$ & .6831 & $\begin{array}{l}.6121 \\
.6121\end{array}$ & .5145 & .4282 & 5.135 & .3929 & .3616 & .3232 & .2934 & & & & & \\
\hline 1.387 & .6831 & $\begin{array}{l}.6121 \\
.6121\end{array}$ & 5145 & .4282 & 6.494 & .3929 & .3616 & .3232 & 2672 & & & & & \\
\hline 1,404 & .6831 & $\begin{array}{l}.6121 \\
.6121\end{array}$ & 5145 & -4282 & 6.897 & .3929 & .3616 & .3232 & .2385 & & & & & \\
\hline 1,422 & $\begin{array}{l}.6831 \\
.6831\end{array}$ & .6121 & .5145 & 4282 & $\begin{array}{l}7.353 \\
7874\end{array}$ & & $\begin{array}{l}2461 \\
2461\end{array}$ & $\begin{array}{l}.2390 \\
.2390\end{array}$ & $\begin{array}{l}.2334 \\
.2334\end{array}$ & & & & & \\
\hline $\begin{array}{l}1.441 \\
t .460\end{array}$ & $\begin{array}{l}.6831 \\
.6831\end{array}$ & .6121 & 5145 & .4282 & & $\$ 19$ & & & & & & & & \\
\hline
\end{tabular}


FIGURE C-7

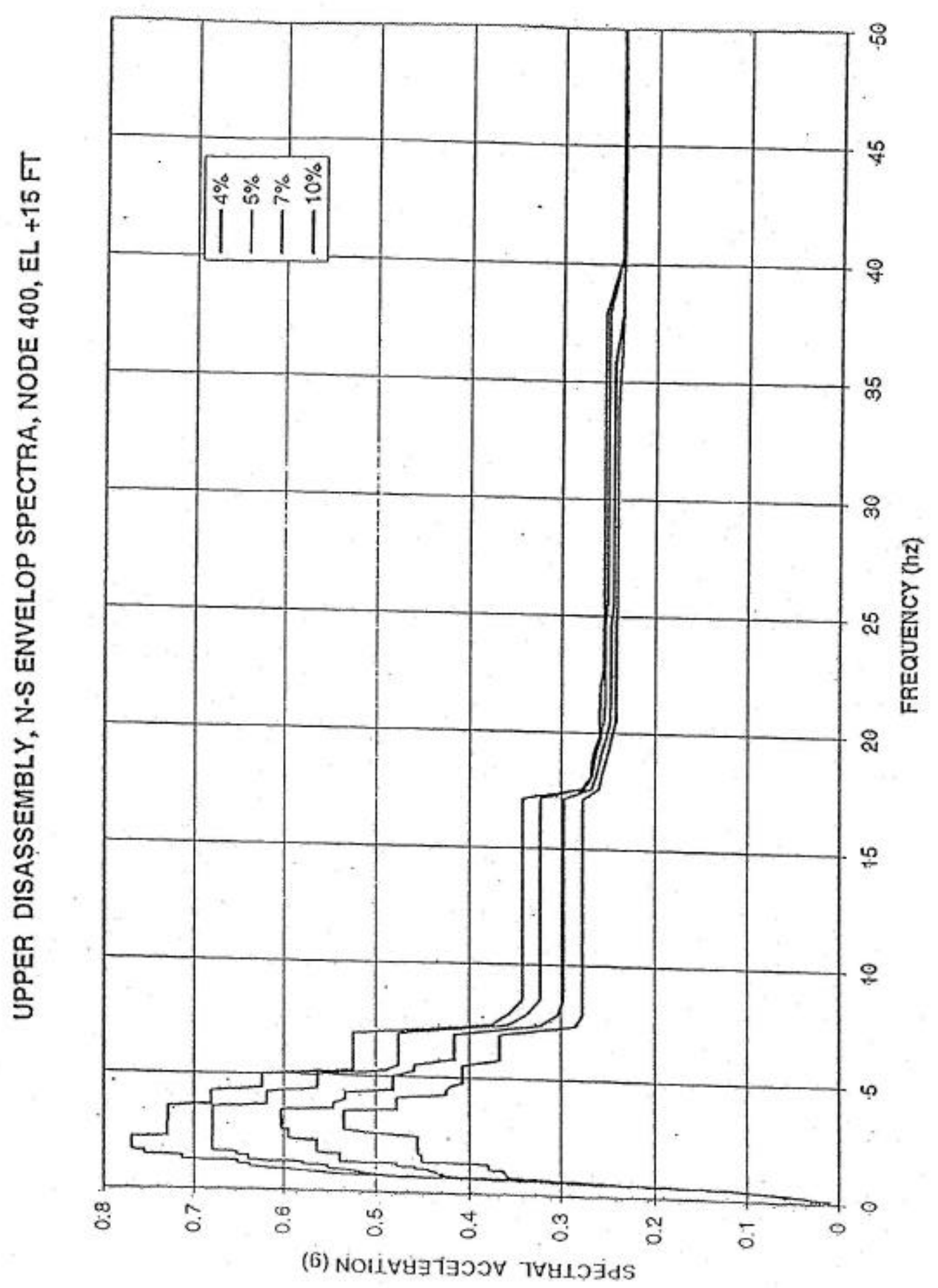


TABLE C-7

Upper Disassembly, envelop spectra, N-S, node $400, \mathrm{EL}+15$, broadened $15 \%$

\begin{tabular}{|c|c|c|c|c|c|c|c|c|c|c|c|c|c|c|}
\hline uency & $4 \%$ & $5 \%$ & $7 \%$ & $10 \%$ & Frequency & $4 \%$ & $5 \%$ & $7 \%$ & $10 \%$ & Frcquency & $4 \%$ & $5 \%$ & $7 \%$ & $10 \%$ \\
\hline .100 & .0105 & .0104 & .0102 & .0100 & 1.479 & .7544 & .6399 & .5390 & .4501 & 8.475 & 3415 & .3224 & .2975 & .2772 \\
\hline .104 & .0105 & .0104 & .0102 & .0101 & L.499 & .7544 & .6533 & .5390 & .4501 & 9.174 & 3415 & 3224 & .2975 & .2772 \\
\hline .108 & .0112 & .0112 & .0111 & .0110 & 1.520 & .7544 & .6533 & .5390 & .4501 & 10.000 & .3415 & .3224 & .2975 & .2772 \\
\hline .112 & .0124 & .0123 & .0120 & .0118 & 1.541 & .7544 & .6533 & .5390 & .4501 & 10.000 & .3415 & .3224 & .2975 & .2772 \\
\hline .117 & .0135 & .0133 & .0129 & .0125 & L.562 & .7544 & .6533 & .5390 & .4501 & 10,163 & .3415 & .3224 & .2975 & .2772 \\
\hline .122 & .0157 & .0154 & .0148 & .0141 & 1.585 & .7544 & .6533 & .5390 & .4501 & 10,331 & .3415 & 3224 & .2975 & .2772 \\
\hline .128 & .0297 & .0278 & .0247 & .0165 & 1.608 & .7544 & .6533 & .5390 & .4501 & 10.504 & .3415 & 3224 & .2975 & .2772 \\
\hline 134 & .0297 & .0278 & .0247 & .0189 & 1.631 & .7544 & .6533 & .5390 & .4501 & 10.684 & 3415 & 3224 & .2975 & .2772 \\
\hline .140 & $.0297^{\circ}$ & .0278 & .0247 & .0209 & 1.656 & .7689 & .6800 & .5640 & .4543 & 10.870 & .3415 & .3224 & .2975 & .2772 \\
\hline .148 & .0297 & .0278 & .0247 & .0215 & 1.681 & .7689 & .6800 & .5640 & .4543 & 11.062 & .3415 & 3224 & .2975 & .2772 \\
\hline .156 & .0297 & .0278 & .0247 & .0217 & 1.706 & .7689 & .6800 & .5640 & .4543 & 11.261 & 3415 & 3224 & .2975 & .2772 \\
\hline .166 & .0298 & .0286 & .0266 & .0243 & 1.733 & .7689 & .6800 & .5640 & .4543 & 11.468 & 3415 & 3224 & .2975 & $27 / 2$ \\
\hline 176 & .0347 & .0332 & .0306 & .0275 & 1.761 & .7689 & .6800 & .5640 & .4543 & 11.682 & .3415 & .3224 & .2975 & .2772 \\
\hline .188 & .0359 & .0344 & .0318 & .0298 & 1.789 & .7689 & .6800 & .5640 & .4543 & 11.905 & .3415 & 3224 & .2975 & .2772 \\
\hline .202 & .0596 & .0554 & .0487 & .0328 & 1.818 & .7689 & .6800 & .5640 & .4543 & 12.136 & 3415 & 3224 & .2975 & .2772 \\
\hline .217 & .0596 & .0554 & .0487 & .0385 & 1.848 & .7689 & .6800 & .5640 & .4543 & 12.376 & .3415 & 3224 & .2975 & .2772 \\
\hline .236 & .0596 & .0554 & .0487 & .0418 & 1.880 & .7689 & .6800 & .5640 & .4543 & 12,626 & .3415 & .3224 & .2975 & .2772 \\
\hline .258 & .0596 & .0554 & .0487 & .0461 & 1.912 & .7689 & .6800 & .5640 & .4543 & 12.887 & .3415 & .3224 & .2975 & .2772 \\
\hline .284 & 0596 & .0554 & .0515 & .0507 & 1.946 & .7689 & .6800 & .5640 & .4543 & 13.158 & .3415 & .3224 & .2975 & .2772 \\
\hline $316^{\circ}$ & .0849 & .0785 & .0687 & .0591 & 1.980 & .7689 & .6800 & .5640 & .4548 & 13.441 & .3415 & 3224 & .2975 & .2772 \\
\hline .357 & .0886 & .0835 & .0757 & .0726 & 2.016 & .7689 & .6800 & .5640 & .4548 & 13.736 & .3415 & .3224 & .2975 & .2772 \\
\hline .410 & .1200 & .1133 & .1051 & .0990 & 2.053 & .7689 & .6800 & .5640 & .4548 & 14.045 & 3415 & .3224 & .2975 & .2772 \\
\hline .481 & .2191 & .2084 & .1899 & .1687 & 2.092 & .7689 & .6800 & .5640 & .4548 & 14.368 & 3415 & .3224 & .2975 & .2772 \\
\hline .581 & 3571 & .3270 & .2970 & .2600 & 2.132 & .7689 & .6800 & .5640 & .4548 & 14.706 & 3415 & .3224 & .2975 & .2772 \\
\hline .735 & .5311 & .4850 & .4233 & 3575 & 2.174 & .7689 & .6800 & .5640 & .4548 & 15.060 & 3415 & .3224 & .2975 & .2772 \\
\hline 1.000 & .6384 & .5520 & .4443 & 3637 & 2.217 & .7689 & .6800 & .5949 & .4548 & 15.432 & 3415 & .3224 & .2975 & .2772 \\
\hline 1.000 & .6384 & .5520 & .4443 & .3637 & 2.262 & .7302 & .6796 & .5949 & .4548 & 23 & 3415 & .3224 & .2975 & .2772 \\
\hline 1.009 & .6384 & .5520 & .4443 & .3637 & 2.309 & .7302 & .6796 & .5949 & .4548 & 16.234 & 3415 & 3224 & .2975 & .2772 \\
\hline 1.018 & .6384 & 5520 &, 4443 & 3637 & 58 & .7302 & .6796 & .5949 & .4548 & 16.667 & 3415 & .3224 & .2975 & .2772 \\
\hline 1.028 & .6384 & .5520 & .4573 & .3637 & 2.410 & .7302 & .6796 & .5949 & .4548 & 17,123 & 3415 & 3224 & .2975 & .2772 \\
\hline 1.037 & .6384 & 5520 & .4573 & 3793 & 463 & .7302 & .6796 & .5949 & .4718 & 17.606 & .2782 & .2745 & .2684 & .2772 \\
\hline 1.047 & .6384 & .5520 & .4573 & .3793 & 2.519 & .7302 & .6796 & .5949 & .4982 & 18.116 & .2691 & .2676 & .2636 & .2573 \\
\hline 1.057 & .6384 & .5520 & .4573 & .3793 & 2.577 & .7302 & .6796 & .5949 & 5147 & 18.657 & .2681 & .2652 & .2602 & .2538 \\
\hline 1.067 & .6384 & .5520 & .4573 & .3793 & 639 & .7302 & .6796 & .6032 & .5347 & 19.231 & .2654 & .2621 & .2565 & .2501 \\
\hline 1.078 & .6384 & .5520 & .4573 & .3793 & 703 & .7302 & .6796 & .6032 & .5347 & 41 & .2596 & .2572 & .2525 & .2466 \\
\hline 1.088 & .6384 & .5520 & .4573 & .3793 & 770 & .7302 & .6796 & .6032 & .5347 & 20.492 & .2595 & .2549 & .2484 & .2423 \\
\hline 1.099 & .6384 & .5520 & .1573 & 3793 & 2.841 & .7302 & .6796 & .6032 & .5347 & 1.186 & $.2591^{\circ}$ & .2541 & .2480 & .2421 \\
\hline 1.110 & .6384 & .5520 & .4573 & .3793 & 15 & .7290 & .6796 & .6032 & .5347 & 30 & .2582 & .2541 & .2480 & .2421 \\
\hline 1.121 & .6519 & .5790 & .4778 & 3793 & .994 & .7290 & .6796 & .6032 & .5347 & 27 & .2550 & .2541 & .2480 & .2421 \\
\hline 1.133 & .6519 & .5790 & .4778 & .3793 & 077 & .7290 & .6796 & .6032 & .53 & 23.585 & .2550 & .2541 & .2480 & .2421 \\
\hline 1.144 & .6519 &, 5790 & 4778 & 3793 & 65 & .7290 & .6796 & .6032 & .534 & 10 & .2550 & .2541 & .2480 & .2421 \\
\hline 1.156 & .6519 & .5790 & .4778 & 3793 & 257 & .7290 & .6796 & .6032 & .5347 & 10 & $.255 Q$ & .2509 & .2453 & .2410 \\
\hline 1,168 & .6519 & .5790 & .4778 & 3793 & 356 & .7290 & .6796 & .6032 & .5347 & 26.596 & $.2550^{\circ}$ & .2509 & .2453 & .2410 \\
\hline 1.181 & .6519 & .5790 & .4778 & 3793 & 60 & .7290 & .6796 & .6032 & .5317 & 27.778 & .2550 & .2509 & .2453 & 2410 \\
\hline 1.193 & .6519 & .5790 & .4778 & .3793 & 571 & .7290 & .6796 & .5459 & .4779 & 70 & .2550 & .2509 & .2453 & .2410 \\
\hline 1.206 & .6519 & .5790 & .4778 & .3793 & 3.690 & .6815 & .6185 & .5459 & .4779 & 30.488 & .2550 & .2509 & .2453 & .2410 \\
\hline 1.220 & .6519 & .5790 & .4778 & .3793 & 3.817 & .6815 & .6185 & .5459 & .4779 & 32,051 & .2550 & .2509 & .2453 & .2410 \\
\hline 1.233 & .6519 & .5790 & .4778 & .3793 & 3.953 & .6815 & .6185 & .5331 & .4779 & 33.784 & .2550 & .2509 & .2453 & .2410 \\
\hline 1.247 & .7134 & .6399 & .4778 & .3805 & 4.098 & .6815 & .6185 & .5331 & .4779 & 35.714 & .2550 & .2509 & .2453 & .2385 \\
\hline 1.261 & .7134 & .6399 & .5390 & .3829 & 4.255 & .6815 & .6185 & .5331 & .424 & 37.879 & .2550 & .2509 & .2366 & .2359 \\
\hline 1.276 & .7134 & .6399 & .5390 & .3912 & 4.425 & .6245 & .5633 & .4819 & .4243 & 40.323 & .2372 & .2367 & .2362 & .2357 \\
\hline 1.290 & .7134 & 6399 & .5390 & 3978 & 4.608 & .6245 & .5633 & .4819 & .4192 & 43.103 & .2372 & .2367 & .2362 & .2357 \\
\hline 1.305 & .7134 & .6399 & .5390 & .4041 & 4.808 & .6245 & .5633 & .4819 & .407 & 46.296 & .2372 & .2367 & .2362 & .2357 \\
\hline 1.321 & .7134 & .6399 & .5390 & .4501 & 5.025 & .6245 & .5633 & .4819 & .4071 & 50.000 & .2372 & .2370 & .2369 & .2366 \\
\hline 1.337 & .7134 & .6399 & .5390 & .4501 & 5.263 & .5243 & .4898 & .4580 & .4071 & & & & & \\
\hline 1.353 & .7134 & .6399 & 5390 & .4501 & 5.525 & .5243 & .4758 & .4580 & .407 & & & & & \\
\hline 1.370 & .7134 & .6399 & .5390 & .4501 & 5.814 & .5243 & .4758 & .4153 & .366 & & & & & \\
\hline 1.387 & .7134 & .6399 & .5390 & .4501 & 6.135 & .5243 & .4758 & .4153 & .3669 & & & & & \\
\hline 1.404 & .7134 & .6399 & .5390 & .4501 & 6.494 & .5243 & .4758 & .4153 & .366 & & & & & \\
\hline 1.422 & .7134 & .6399 & .5390 & $.450 t$ & 6.897 & .5243 & .4758 & .4153 & .3669 & & & & & \\
\hline & $\begin{array}{l}7162 \\
7162\end{array}$ & $\begin{array}{l}.6399 \\
6390\end{array}$ & $\begin{array}{l}5390 \\
5390\end{array}$ & 4501 & & & .3565 & .3226 & .2847 & & & & & \\
\hline 460 & .7162 & & 5390 & & 874 & 52 & .3360 & 3031 & בר7 & & & & & \\
\hline
\end{tabular}


FIGURE C-8

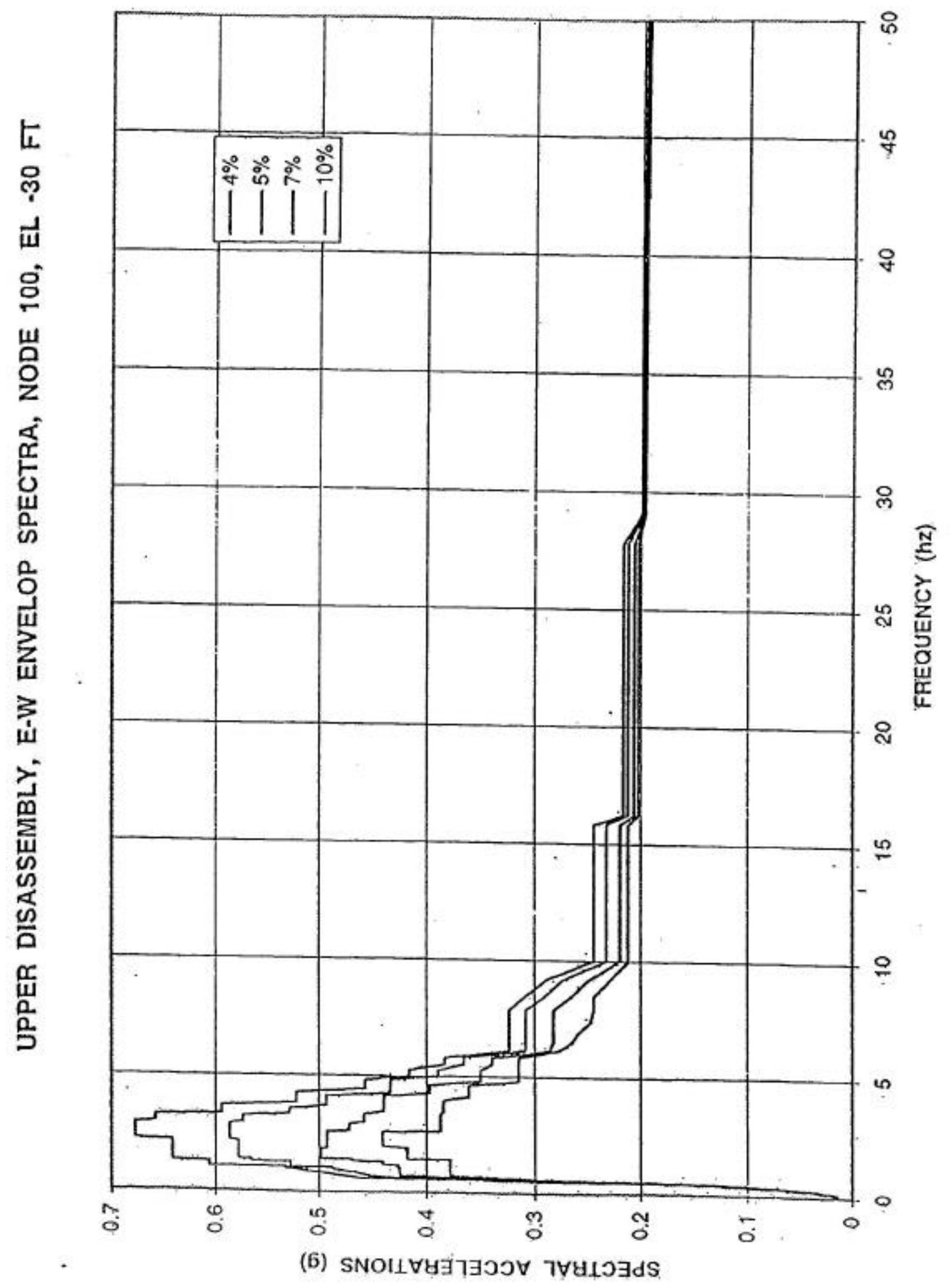




\section{TABLE C-8}

Upper Disassembly, envelop spectra, E-W , node 100, EL-30, broadened $15 \%$

\begin{tabular}{|c|c|c|c|c|c|c|c|c|c|c|c|c|c|c|}
\hline irequency & $4 \%$ & $5 \%$ & $7 \%$ & $10 \%$ & Frequency & $4 \%$ & $5 \%$ & $7 \%$ & $10 \%$ & Frequency & $4 \%$ & $s \%$ & $7 \%$ & $10 \%$ \\
\hline .100 & .0149 & .0146 & .0142 & .0138 & 1.479 & .6416 & .5778 & .4979 & .4184 & 8.475 & .3084 & .2890 & .2645 & .2441 \\
\hline .104 & .0175 & .0169 & .0159 & .0150 & 1.499 & .6416 & .5778 & .4979 & .4184 & 9.174 & .2883 & .2732 & .2492 & .2291 \\
\hline .108 & .0175 & .0169 & .0159 & .0150 & 1.520 & .6416 & .5778 & .4979 & .4184 & 10.000 & .2439 & .2322 & .2195 & .2117 \\
\hline .112 & .0175 & .0169 & .0159 & .0150 & 1.541 & .6416 & .5778 & .4979 & .4184 & 10,000 & .2439 & .2322 & .2195 & .2117 \\
\hline .117 & 0175 & .0169 & .0159 & .0150 & 1.562 & .6416 & .5778 & .4979 & .4184 & 10.163 & .2439 & .2322 & .2195 & .2117 \\
\hline .122 & .0175 & .0169 & .0159 & .0150 & 1.585 & .6416 & .5778 & .4979 & .4184 & 10.331 & .2439 & .2322 & .2195 & .2117 \\
\hline .128 & .0175 & .0169 & .0159 & .0150 & 1.608 & .6416 & 5778 & .4979 & .4184 & 10.504 & .2439 & .2322 & .2195 & .2117 \\
\hline .134 & .0175 & .0169 & .0159 & .0160 & 1.631 & .6416 & .5778 & .4979 & .4184 & 10.684 & .2439 & .2322 & .2195 & .2117 \\
\hline .140 & .0175 & .0171 & .0170 & .0171 & 1.656 & .6416 & .5778 & .4979 & .4184 & 10.870 & .2439 & .2322 & .2195 & .2117 \\
\hline .148 & .0186 & .0201 & .0197 & .0181 & $1.68 \mathrm{I}$ & .6416 & .5778 & .4979 & .4184 & 11.062 & .2439 & .2322 & .2195 & .2117 \\
\hline .156 & .0197 & .0201 & .0197 & .0189 & 1.706 & .6416 & .5778 & .4979 & .4184 & 11,261 & .2439 & .2322 & .2195 & .2117 \\
\hline .166 & .0204 & .0201 & .0197 & .0193 & 1.733 & .6416 & 5778 & .4979 & .4184 & 11.468 & .2439 & .2322 & .2195 & .2117 \\
\hline .176 & .0204 & .0201 & .0197 & .0194 & 1.761 & .6416 & 5778 & .4979 & .4184 & 11.682 & .2439 & .2322 & .2195 & .2117 \\
\hline .188 & .0352 & .0339 & .0319 & .0238 & 1.789 & .6416 & .5778 & .4979 & .4184 & 11.905 & .2439 & .2322 & .2195 & .2117 \\
\hline .202 & .0352 & .0339 & .0319 & .0267 & 1.818 & .6416 & .5778 & .4979 & .4184 & 12.136 & .2439 & .2322 & .2195 & .2117 \\
\hline .217 & .0352 & .0339 & .0319 & .0304 & 1.848 & .6416 & 5778 & .4979 & .4184 & 12.376 & .2439 & .2322 & .2195 & .2117 \\
\hline .236 & .0352 & .0339 & .0319 & .0333 & 1.880 & .6416 & .5778 & .4919 & .4184 & 12.626 & .2439 & .2322 & .2195 & .2117 \\
\hline .258 & .0630 & .0599 & .0474 & .0442 & 1.912 & .6416 & .5778 & .4919 & .4184 & 12.887 & .2439 & .2322 & .2195 & .2117 \\
\hline .284 & .0630 & .0599 & .0550 & .0499 & 1.946 & .6416 & .5778 & .4919 & 4405 & 13.158 & .2439 & .2322 & .2195 & .2117 \\
\hline .316 & .0630 & .0599 & .0572 & .0538 & 1.980 & .6416 & .5778 & .4919 & .4405 & $13.44 \mathrm{I}$ & .2439 & .2322 & .2195 & .2117 \\
\hline .357 & .0915 & .0876 & .0820 & .0765 & 2.016 & .6416 & .5778 & .4919 & .4405 & 13.736 & .2439 & .2322 & .2195 & .2117 \\
\hline .410 & .1280 & .1200 & .1093 & .1007 & 2.053 & .6416 & 5778 & .4919 & .4405 & 14.045 & .2439 & .2322 & .2195 & .2117 \\
\hline .481 & .2129 & .2017 & .1848 & .1676 & 2.092 & .6416 & .5778 & .4919 & .4405 & 14.368 & .2439 & .2322 & .2195 & .2117 \\
\hline .581 & .4609 & .4157 & 3475 & .2876 & 2.132 & .6416 & .5778 & .4919 & .4405 & 14.706 & .2439 & .2322 & .2195 & .2117 \\
\hline .735 & .4833 & .4554 & .4249 & 3779 & 2,174 & .6416 & .5778 & .4919 & .4405 & 15.060 & .2439 & .2322 & .2195 & .2117 \\
\hline 1.000 & 5321 & .4872 & .4249 & 3779 & 2.217 & .6772 & .5864 & .4919 & .4405 & 15,432 & .2439 & .2322 & .2195 & .2117 \\
\hline 1.000 & .5321 & .4872 & .4249 & .3779 & 2.262 & .6772 & .5864 & .4919 & .4405 & 15.823 & .2439 & .2322 & .2195 & .2117 \\
\hline 1.009 & .5321 & .4872 & .4249 & 3779 & 2.309 & .6772 & .5864 & .4919 & .4405 & 16.234 & .2169 & .2123 & 2195 & .2117 \\
\hline 1.018 & .5321 & .4872 & .4249 & 3779 & 2358 & .6772 & .5864 & .4919 & .4405 & 16.667 & .2169 & .2123 & .2066 & .2023 \\
\hline 1.028 & .5321 & .4872 & .4249 & 3779 & 2.410 & .6772 & .5864 & .4919 & .4405 & 17.123 & .2169 & .2123 & .2066 & .2023 \\
\hline 1.037 & .5321 & .4872 & .4265 & 3779 & 2,463 & .6772 & .5864 & .4919 & .4405 & 17.606 & .2169 & .2123 & .2066 & .2023 \\
\hline 1.047 & .5321 & .4872 & .4265 & 3779 & 2.519 & .6772 & 5864 & .4919 & .4405 & 18. & .2169 & .2123 & .2066 & .2023 \\
\hline 1.057 & .5321 & 5271 & .4265 & 3779 & 2.577 & .6772 & 5864 & .4919 & .4405 & 18.657 & .2169 & .2123 & .2066 & .2023 \\
\hline 1.067 & .6064 &.$\$ 271$ & .4265 & 3779 & 2.639 & .6772 & .5864 & .4716 & 3873 & 19.231 & .2169 & .2123 & .2066 & .2023 \\
\hline 1.078 & .6064 & 5271 & .4265 & 3779 & 2.703 & .6772 & .5864 & .4716 & 3873 & 19.841 & .2169 & .2123 & .2066 & .2023 \\
\hline 1.088 & .6064 & 5271 & .4265 & 3779 & 2.770 & .6772 & .5864 & .4716 & 3873 & 20.492 & .2169 & .2123 & .2066 & .2023 \\
\hline 1.099 & .6064 & 5271 & .4265 & .3779 & 2.841 & .6772 & .5864 & .4716 & 3873 & 21.186 & .2169 & .2123 & .2066 & .2023 \\
\hline 1.110 & .6064 & 5271 & .4265 & 3779 & 2.915 & .6772 & .5864 & .4716 & .3873 & 21.930 & .2169 & .2123 & .2066 & .2023 \\
\hline 1.121 & .6064 & .5271 & .4265 & 3779 & 2.994 & .6579 & .5740 & .4577 & 3873 & 22.727 & .2169 & .2123 & .2066 & .2023 \\
\hline 1.133 & .6064 & .5271 & .4265 & .3779 & 3.077 & .6579 & .5740 & .4577 & 38 & 23.585 & .2169 & .2123 & .2066 & .2023 \\
\hline 1.144 & .6064 & .5271 & .4265 & .3779 & 3.165 & .6579 & .5740 & .4577 & 3873 & 24.510 & .2169 & .2123 & .2066 & .2023 \\
\hline 1.156 & .6064 & .5271 & .4265 & .3779 & 3.257 & .6579 &.$\$ 740$ & .4577 & .3873 & 25.510 & .2169 & .2123 & .2066 & .2023 \\
\hline 1.168 & .6064 & 5271 & .4407 & 3779 & 3.356 & .5942 & .5283 & .4577 & 3873 & 26.596 & .2169 & .2123 & .2066 & .2023 \\
\hline L. 181 & .6064 & .5271 & .4407 & .3779 & 3.460 & .5942 & .5283 & .4391 & 3844 & 27.778 & .2169 & .2123 & .2066 & .2023 \\
\hline 1.193 & .6064 & .5271 & .4407 & .3779 & 3.571 & .5942 & .5283 & .4391 & .3844 & 29.070 & .1997 & .1983 & .1967 & .1963 \\
\hline 1.206 & .6064 & .5271 & .4407 & .3779 & 3.690 & .5942 & .4930 & .4391 & .3844 & 30.488 & .1997 & .1983 & 1967 & .1957 \\
\hline 1.220 & .6064 & 5271 & .4407 & .3779 & 3.817 & .5216 & .4930 & .4391 & .3844 & 32.051 & .1997 & .1983 & .1967 & .1957 \\
\hline 1.233 & .6064 & .5271 & .4407 & .3779 & 3.953 & .5216 & .4930 & .4391 & 3844 & 33.784 & .1997 & .1983 & .1967 & .1957 \\
\hline 1.247 & .6064 & 5271 & .4407 & .3779 & 4.098 & .5216 & .4930 & .4391 & .3611 & 35. & .1997 & .1983 & .1967 & .1957 \\
\hline 1.261 & .6064 & 5271 & .4407 & 3779 & 4.255 & 5216 & .4332 & .3977 & 3611 & 37.879 & .1997 & .1983 & .1967 & .1957 \\
\hline 1.276 & .6064 & .5271 & .4407 & .3779 & 4.425 & .4570 & .4332 & 3977 & .3611 & 40.323 & .1997 & .1983 & 1967 & .1957 \\
\hline 1.290 & .6064 & .5271 & .4407 & .3779 & 4.608 & .4570 & .4332 & .3977 & 3611 & 43.103 & .1969 & 1965 & .1958 & .1952 \\
\hline 1.305 & .6416 & .5657 & .4407 & .3779 & 4.808 & .4570 & .4332 & 3504 & 3156 & 46.296 & .1969 & .1965 & .1958 & .1952 \\
\hline 1.321 & .6416 & .5657 & .4689 & .3779 & 5.025 & .4165 & .3893 & .3504 & .3156 & 50.000 & .1969 & .1965 & .1958 & .1946 \\
\hline 1.337 & .6416 & .5657 & 4689 & 3779 & 5.263 & .4165 & .3893 & .3504 & 3156 & & & & & \\
\hline 1.353 & .6416 & .5657 & -4689 & .3779 & 5.525 & .3829 & .3655 & .3402 & .3156 & & & & & \\
\hline 1.370 & .6416 & .5657 & -4689 & 3779 & 5.814 & 3829 & 3655 & .3402 & .3156 & & & & & \\
\hline 1.387 & .6416 & .5657 & .4689 & 3779 & 6.135 & .3245 & .3085 & 2850 & .2767 & & & & & \\
\hline 1.404 & .6416 & .5778 & 4689 & .3779 & 6.494 & .3245 & .3085 & .2816 & .2646 & & & & & \\
\hline 1.422 & .6416 & .5778 & -4979 & .4184 & 6.897 & 3245 & .3085 & .2816 & .2588 & & & & & \\
\hline$\{.44\}$ & .6416 & .5778 & 4979 & .4184 & 7.353 & 3245 & 3085 & .2816 & .2476 & & & & & \\
\hline 1.460 & 6416 & .5778 & 4979 & 4184 & 7.874 & 3245 & 3085 & .2816 & .2443 & & & & & \\
\hline
\end{tabular}


WSRC-TR-97-00289

Revision 0

Page C17 of C30

FIGURE C-9

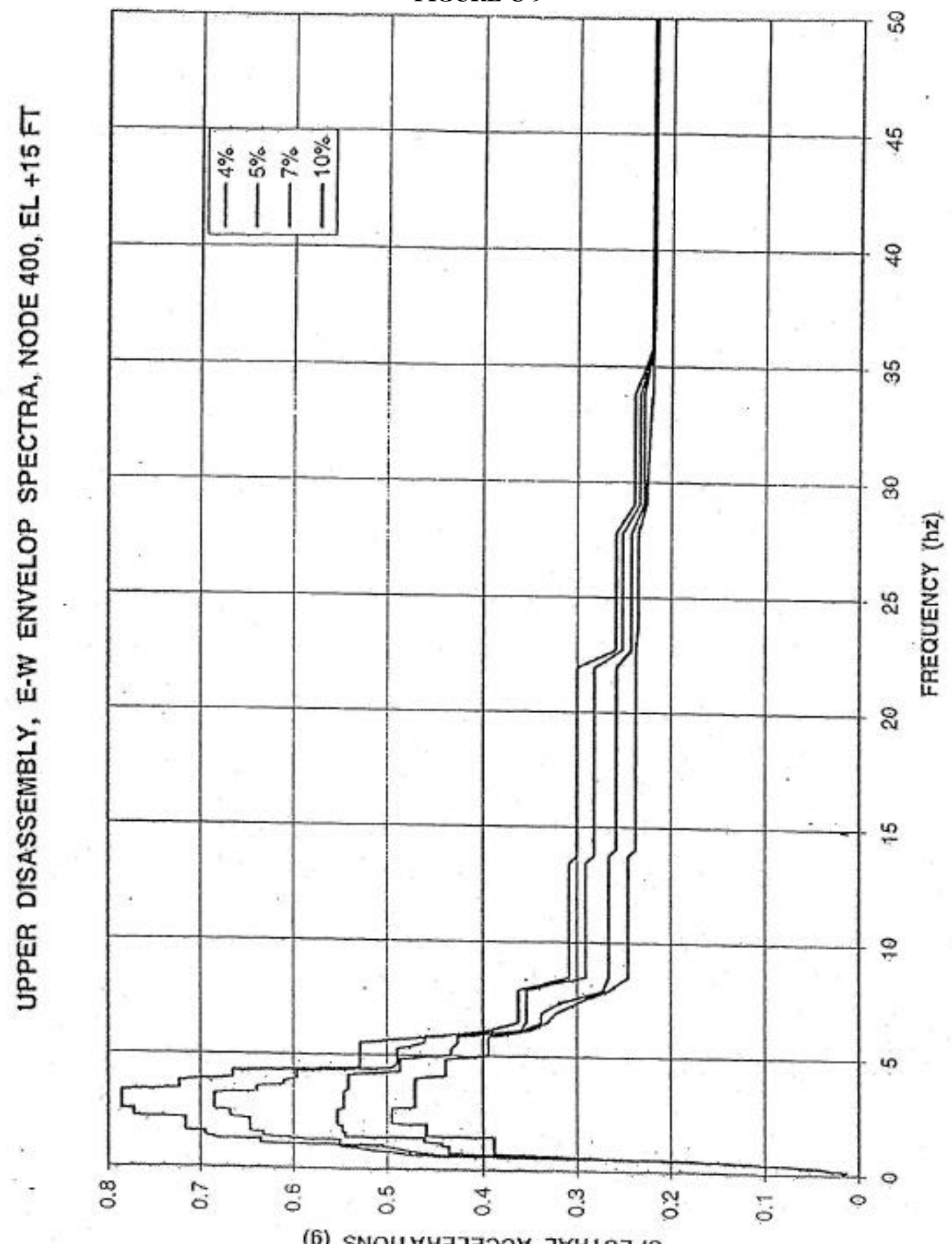

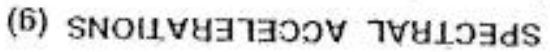




\section{TABLE C-9}

Upper Disassembly, envelop spectra, E-W , node $400, \mathrm{EL}+15$, broadened $15 \%$

\begin{tabular}{|c|c|c|c|c|c|c|c|c|c|c|c|c|c|c|}
\hline equency & y $\quad 4 \%$ & $5 \%$ & $7 \%$ & $10 \%$ & Frequency & $4 \%$ & $5 \%$ & $7 \%$ & $10 \%$ & Frequency & $4 \%$ & $s \%$ & $7 \%$ & $10 \%$ \\
\hline .100 & .0150 & .0147 & .0142 & .0139 & 1.479 & .6952 & .6324 & .5452 & .4598 & 8.475 & 3079 & .2905 & .2665 & 2460 \\
\hline .104 & .0176 & .0170 & .0160 & .0139 & l.499 & .6952 & .6324 & .5452 & .4598 & 9.174 & .3079 & .2905 & .2665 & .2460 \\
\hline .108 & .0176 & .0170 & .0160 & .0142 & 1.520 & .6952 & .6324 & .5452 & .4598 & 10.000 & .3079 & .2905 & .2665 & .2460 \\
\hline .112 & .0176 & .0170 & .0160 & .0144 & 1.541 & .6952 & .6324 & .5452 & .4598 & 10.000 & .3079 & .2905 & .2665 & .2460 \\
\hline .117 & .0176 & .0170 & .0160 & .0149 & 1.562 & .6952 & .6324 & .5452 & .4598 & 10.163 & .3079 & .2905 & .2665 & .2460 \\
\hline .122 & .0176 & .0170 & .0160 & .0151 & 1.585 & .6952 & .6324 & .5452 & .4598 & 10.331 & .3079 & .2905 & .2665 & .2460 \\
\hline .128 & .0176 & .0170 & .0160 & .0151 & 1.608 & .7164 & .6465 & .5477 & .4598 & 10.504 & .3079 & .2905 & .2665 & .2460 \\
\hline .134 & .0176 & .0170 & .0160 & .0163 & 1.631 & .7164 & .6465 & .5477 & .4598 & 10.684 & .3079 & .2905 & .2665 & .2460 \\
\hline .140 & .0176 & .0173 & .0173 & .0174 & 1.656 & .7164 & .6465 & .5477 & .4598 & 10.870 & .3079 & .2905 & .2665 & .2460 \\
\hline .148 & .0188 & .0204 & .0200 & .0197 & 1.681 & .7164 & .6465 & .5477 & .4598 & 11.062 & .3079 & .2905 & .2665 & .2460 \\
\hline .156 & .0199 & .0204 & .0200 & .0197 & 1.706 & .7164 & .6465 & .5477 & .4598 & 11.261 & 3079 & .2905 & .2665 & .2460 \\
\hline .166 & .0206 & .0204 & .0200 & .0197 & 1.733 & .7164 & .6465 & .5477 & .4598 & 11.468 & 3079 & .2905 & .2665 & .2460 \\
\hline .176 & .0355 & .0204 & .0200 & .0197 & 1.761 & .7164 & .6465 & .5477 & .4598 & 11.682 & .3079 & .2905 & .2665 & .2460 \\
\hline .188 & .0355 & .0341 & .0321 & .0240 & 1.789 & .7164 & .6465 & .5477 & .4598 & 11.905 & 3079 & .2905 & .2665 & .2460 \\
\hline .202 & .0355 & .0341 & .0321 & .0271 & 1.818 & .7164 & .6465 & .5477 & .4598 & 12.136 & .3079 & .2905 & .2665 & .2460 \\
\hline .217 & .0355 & .0341 & .0321 & .0306 & 1.848 & .7164 & .6465 & .5477 & .4598 & 12.376 & .3079 & .2905 & .2665 & .2460 \\
\hline .236 & .0355 & .0341 & .0321 & .0336 & 1.880 & .7164 & .6465 & .5477 & .4598 & 12.626 & .3079 & .2905 & .2665 & .2460 \\
\hline .258 & .0630 & .0599 & .0476 & .0443 & 1.912 & .7164 & .6465 & .5527 & .4598 & 12.887 & .3079 & .2905 & .2665 & .2460 \\
\hline .284 & .0630 & .0599 & .0551 & .0500 & 1.946 & .7164 & .6465 & .5527 & .4598 & 13.158 & .3079 & .2905 & .2665 & .2460 \\
\hline 316 & .0630 & .0599 & .0574 & .0542 & 1.980 & .7164 & .6465 & .5527 & .4958 & 13.441 & .3079 & .2905 & .2665 & .2460 \\
\hline 357 & .0923 & .0885 & .0829 & .0775 & 2.016 & .7164 & .6465 & .5532 & .4958 & 13.736 & .3002 & .2822 & .2665 & .2460 \\
\hline .410 & .1296 & .1216 & .1109 & .1024 & 2.053 & .7164 & .6465 & .5532 & .4958 & 14.045 & .3002 & .2822 & .2588 & .2381 \\
\hline .481 & .2155 & .2042 & .1873 & .1702 & 2.092 & .7164 & .6465 & .5532 & .4958 & 14.368 & .3002 & .2822 & .2588 & .2381 \\
\hline .581 & .4675 & .4217 & .3524 & .2921 & 2.132 & .7164 & .6465 & .5532 & .4958 & 14.706 & .3002 & .2822 & .2588 & .2381 \\
\hline .735 & .4950 & .4671 & .4356 & 3879 & 2.174 & .7164 & .6465 & .5532 & .4958 & 15.060 & .3002 & .2822 & .2588 & .2381 \\
\hline 1.000 & .5518 & .5051 & .4356 & 3879 & 2.217 & .7723 & .6683 & .5532 & .4958 & 15.432 & .3002 & .2822 & .2588 & .2381 \\
\hline 1.000 & .5518 & .5051 & .4356 & .3879 & 2.262 & .7723 & .6683 & .5532 & .4958 & 15.823 & .3002 & .2822 & 2588 & .2381 \\
\hline 1.009 & .5518 & .5051 & .4356 & .3879 & 2.389 & .7723 & .6683 & .5532 & .4958 & 16.234 & .3002 & .2822 & .2588 & .2381 \\
\hline 1.018 & .5518 & .sosi & .4356 & .3879 & 2.358 & .7723 & .6683 & .5532 & .4958 & 16.667 & .3002 & .2822 & .2588 & $.238 \overline{\mathrm{i}}$ \\
\hline 1.028 & .5518 & .5051 & .4356 & 3879 & 2.410 & .7723 & .6683 & .5532 & .4958 & 17.123 & .3002 & .2822 & .2588 & .2381 \\
\hline 1.037 & .5518 & .5051 & .4433 & .3879 & 2.463 & .7723 & .6683 & .5532 & .4958 & 17.606 & .3002 & .2822 & .2588 & .2381 \\
\hline 1.047 & .5518 & .SOS1 & .4433 & .3879 & 2.519 & .7723 & .6683 & .5532 & .4958 & 18.116 & .3002 & .2822 & .2588 & .2381 \\
\hline 1.057 & .5518 & .5503 & .4433 & 3879 & 2.577 & .7856 & .6855 & .5532 & .4958 & 18.657 & .3002 & .2822 & .2588 & .2381 \\
\hline 1.067 & .6353 & .5503 & .4433 & .3879 & 2.639 & .7856 & .6855 & .5468 & .4958 & 19.231 & .3002 & .2822 & .2588 & .2381 \\
\hline 1.078 & .6353 & .5503 & .4433 & .3879 & 2.703 & .7856 & .6855 & .5468 & .4714 & 19.841 & .3002 & .2822 & .2588 & .2381 \\
\hline 1.088 & .6353 & .5503 & .4433 & .3879 & 2.770 & .7856 & .6855 & .5468 & .4714 & 20.492 & .3002 . & .2822 & .2588 & .2381 \\
\hline 1.099 & .6353 & .5503 & .4433 & .3879 & 2.841 & .7856 & .6855 & .5468 & .4714 & 21.186 & $3002^{\circ}$ & .2822 & .2588 & .2381 \\
\hline 1.110 & .6353 & .5503 & .4433 & .3879 & 2.915 & .7856 & .6855 &, 5468 & .4714 & 21.930 & .3002 & .2822 & .2588 & .2381 \\
\hline 1.121 & .6353 & .5503 & .4433 & .3879 & 2.994 & .7856 & .6855 & .5468 & .4714 & 22.727 & .2596 & .2522 & .2588 & .2381 \\
\hline 1.133 & .6353 & 5503 & .4433 & .3879 & 3.077 & .7856 & .6855 & .5468 & .4714 & 23.585 & .2596 & .2522 & .2428 & .2355 \\
\hline L.144 & .6353 & .5503 & .4433 & .3879 & 3.165 & .7856 & .6855 & .5468 & .4714 & 24.510 & .2596 & .2522 & .2428 & .2355 \\
\hline 1.156 & .6353 & .5503 & .4433 & .3879 & 3.257 & .7856 & .6855 & .5468 & .4714 & 510 & .2596 & .2522 & .2428 & .2355 \\
\hline 1.168 & .6353 & .5503 & .4618 & .3879 & 3.356 & .7856 & .6391 & .5468 & .4714 & 26.596 & .2596 & .2522 & .2428 & .2355 \\
\hline 1.181 & .6353 & .5503 & .4618 & 3879 & 3.460 & .7228 & .6391 & .5418 & .4714 & 27.778 & .2596 & .2522 & .2428 & .2355 \\
\hline 1.193 & .6353 & .5503 & .4618 & .3879 & 3.571 & .7228 & .6391 & .5418 & .4714 & 29.070 & .2396 & .2342 & .2297 & .2266 \\
\hline 1.206 & .6353 & .5503 & .4618 & .3879 & 3.690 & .7228 & .6106 & .5418 & .4714 & 30.488 & .2396 & .2342 & .2297 & .2250 \\
\hline 1.220 & .6353 & .5503 & .4618 & .3879 & 3.817 & .7228 & .6106 & .5418 & .4714 & 32.051 & .2396 & .2342 & .2297 & .2234 \\
\hline 1.233 & .6353 & .5503 & .4618 & .3879 & 3.953 & .6653 & .5967 & .5418 & .4714 & 33.784 & .2396 & .2342 & .2297 & .2208 \\
\hline 1.247 & .6353 & .5503 & .4618 & .3879 & 4.098 & .6653 & .5967 & .5418 & .4390 & 35.714 & .2210 & .2203 & .2198 & .2196 \\
\hline 1.261 & .6353 & .5503 & .4618 & .3879 & 4.255 & .6653 & .5967 & .4870 & .4390 & 37.879 & .2210 & .2203 & .2194 & .2186 \\
\hline 1.276 & .6807 & .5503 & .4618 & .3879 & 4.425 & .5287 & .4937 & .4870 & .4390 & 40.323 & .2210 & .2203 & .2194 & .2186 \\
\hline 1.290 & .6807 & .5503 & .4618 & .3879 & 4.608 & .5287 & .4897 & .4870 & .4390 & 43.103 & .2210 & .2203 & .2194 & .2186 \\
\hline 1.305 & .6857 & .6040 & .4618 & .3879 & 4.808 & .5287 & .4897 & .4870 & .4390 & 46.296 & .2210 & .2203 & .2194 & .2186 \\
\hline 1.321 & .6857 & .6040 & .4618 & .3879 & 5.025 & 5287 & .4897 & .4331 & .3936 & 50.000 & .2210 & .2203 & .2194 & .2186 \\
\hline 1.337 & .6857 & .6040 & .4618 & .3879 & 5.263 & .5287 & .4897 & .4331 & .3936 & & & & & \\
\hline 1.353 & .6857 & .6040 & .4618 & .3879 & 5.525 & .5287 & .4614 & .4261 & .3936 & & & & & \\
\hline 1.370 & .6857 & .6040 & .4618 & .3879 & 5.814 & .4494 & .4614 & .4261 & .3936 & & & & & \\
\hline 1.387 & .6857 & .6040 & .4618 & .3879 & 6.135 & .3928 & .3599 & .3576 & 3500 & & & & & \\
\hline 1.404 & .6952 & .6324 & .5452 & 3879 & 6.494 & .3623 & .3529 & .3378 & 3314 & & & & & \\
\hline 1.422 & .6952 & .6324 & .5452 & .3879 & 6.897 & .3623 & .3529 & .3373 & .3208 & & & & & \\
\hline$\{.44\}$ & .6952 & .6324 & .5452 & 4598 & 7.353 & .3623 & 3529 & 3164 & .300 & & & & & \\
\hline 1.460 & .6952 & 6324 & .5452 & $459 \mathrm{~g}$ & 7874 & 3623 & 3529 & 2733 & .2675 & & & & & \\
\hline
\end{tabular}


FIGURE C-10

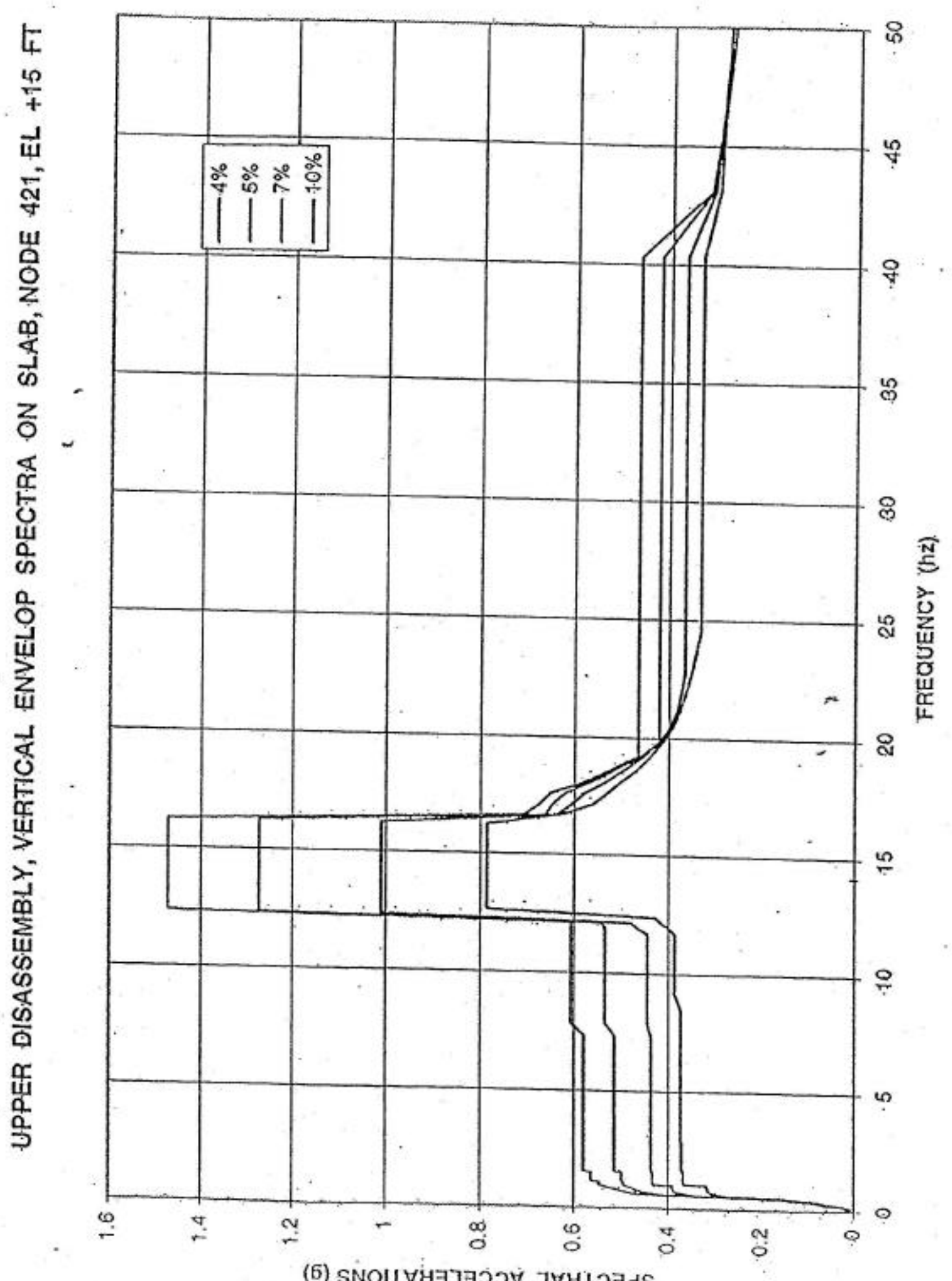

(6) SNOIIV4 
TABLE C-10

Upper Disassembly, Vert Envelop Spectra, ou slab/bcam node, broadened 15\%

\begin{tabular}{|c|c|c|c|c|c|c|c|c|c|c|c|c|c|c|}
\hline equency & $4 \%$ & $5 \%$ & $7 \%$ & $10 \%$ & Prequency & $4 \%$ & $s \%$ & $7 \%$ & $10 \%$ & Proquency & $4 \%$ & $5 \%$ & $7 \%$ & $10 \%$ \\
\hline .100 & .0098 & .0096 & .0092 & .0088 & 1.479 & .5647 & 5008 & .4345 & 3692 & 8.475 & .6062 &, 5356 & .4451 & .3738 \\
\hline .104 & .0098 & .0096 & .0092 & .0094 & 1.499 & .5647 & 5008 & .4345 & 3692 & 9.174 & .6062 & .5356 & -4451 & 3876 \\
\hline .108 & .0100 & .0100 & .0100 & .0103 & 1.520 & .5647 & .5008 & .4345 & 3692 & 10.000 & .6062 & .5356 & 4451 & .3876 \\
\hline .112 & .0109 & .0109 & .0110 & .0111 & 1.541 & .5800 & .5158 & .4345 & 3692 & 10.000 & .6062 & .5356 & .4451 & $: 3876$ \\
\hline .117 & .0119 & .0118 & .0119 & .0121 & 1.562 & .5800 & .5158 & .4378 & 3692 & 10,163 & .6062 & .5356 & .4451 & .3876 \\
\hline .122 & .0130 & .0130 & .0131 & .0132 & 1.585 & 5800 & .5158 & .4378 & 3728 & 10,331 & .6062 & .5356 & $.445 \mathrm{t}$ & .3876 \\
\hline .128 & .0144 & .0113 & .0144 & .0144 & 1.608 & .5800 & .5158 & .4378 & 3728 & 10.504 & .6062 & .5356 & -4451 & .3876 \\
\hline .134 & .0159 & .0159 & .0158 & .0158 & 1.631 & .5800 & .5158 & .4378 & 3728 & 10.684 & .6062 & .5356 & $-445 \mathrm{t}$ & 3876 \\
\hline .140 & .0178 & .0177 & .0175 & .0173 & 1.656 & .5800 & .5158 & .1378 & 3728 & 10.870 & .6062 & .5356 & .4451 & 3876 \\
\hline .148 & .0197 & $.0195^{\circ}$ & .0192 & .0189 & 1.681 & .5800 & .5158 & .4378 & 3728 & 11.062 & .6062 & .5356 & .4451 & .3876 \\
\hline .156 & .0215 & .0212 & .0209 & .0206 & 1.706 & .5800 & .5158 & .4378 & 3728 & 11.261 & .6062 & .5356 & .4451 & 3876 \\
\hline .166 & .0232 & .0231 & .0228 & .0224 & 1.733 & .5800 & 5158 & .4378 & 3728 & 11.468 & .6062 & .5356 & .4451 & 3876 \\
\hline .176 & .0259 & .0257 & .0254 & .0248 & 1.761 & .5800 & .5158 & .4378 & 3728 & 11.682 & .6062 & .5356 & .4451 & 3876 \\
\hline .188 & -0306 & .0302 & .0291 & .0278 & 1.789 &, 5800 & .5158 & .4378 & 3728 & 11.905 & .6062 & .5356 & .4660 & 3984 \\
\hline .202 & .0362 & .0351 & .0331 & .0306 & 1.818 & .5800 & .5158 & .4378 & 3728 & 12.136 & .6062 & .5453 & .4793 & .4157 \\
\hline .217 & .0390 & .0377 & .0355 & .0329 & 1.848 & 5800 & .5158 & .4378 & 3728 & 12.376 & $1.472 \mathrm{t}$ & 1.2725 & 1.0101 & 1. 4302 \\
\hline .236 & .0630 & .0592 & .0436 & .0398 & 1.880 & .5800 & .5158 & .4378 & 3728 & 12.626 & 1.4721 & 1.2725 & 2.0101 & 1.7866 \\
\hline .258 & .0630 & .0592 & .0528 & .0455 & 1.912 & 5800 & .5158 & .4378 & 3728 & 12.887 & 1.4721 & 1.2725 & t.010t & 1.7866 \\
\hline 284 & .0630 & .0592 & .0547 & .0504 & 1.946 & .5800 & .5158 & .4378 & 3728 & 13.158 & 1.4721 & 1.2725 & 1.0101 & .7866 \\
\hline 316 & .0800 & 0754 & .0681 & .0621 & 1.980 & .5800 & .5158 & .4378 & 3728 & 13.441 & 1.4721 & 1.2725 & 1.0101 & .7866 \\
\hline 357 & $.0976^{-}$ & .0942 & .0882 & .0800 & 2.016 & .5800 & .5158 & .4378 & 3728 & 13.736 & 1.4721 & 1.2725 & $t .0101$ & .7866 \\
\hline 410 & .1303 & .1238 & .1130 & .1004 & 2.053 & .5800 & .5158 & .4378 & 3728 & 14.045 & 1.4721 & 1.2725 & L.0101 & 1.7866 \\
\hline .481 & .1558 & .1541 & .1447 & .1272 & 2.092 & .5800 & .5158 & .4378 & 3728 & 14.368 & 1.4721 & 1.2725 & $1.010 \mathrm{t}$ & .7866 \\
\hline 581 & .4657 & .4212 & .3534 & .2865 & 2.132 & .5800 & 5158 & .4378 & .3728 & 14.706 & 1,4721 & 1.2725 & 1.0101 & .7866 \\
\hline .735 & 5032 & .4611 & 3889 & 3152 & 2.174 & .5800 & 5158 & .4378 & 3728 & 15.060 & 1.4721 & 1.2725 & 1.0101 & 1.7866 \\
\hline 1.000 & .5485 & .4969 & 3919 & .3190 & 2.217 & .5800 & .5158 & .4378 & .3728 & 15.432 & 1.4721 & 1.2725 & 1.0101 & .7866 \\
\hline 1.000 & .5485 & .4969 & 3919 & .3190 & 2.262 & .5800 & .5158 & .4378 & 3728 & 15,823 & 1.4721 & 1.2725 & 1.0101 & .7866 \\
\hline 1.009 & .5485 & .4969 & .4345 & .3190 & 2.309 & .5800 & 5158 & .4378 & 3728 & 16.234 & 1.4721 & 1.2725 & 1.0101 & 1.7866 \\
\hline 1.018 & .5485 & .4969 & .4345 & .3692 & 2.358 & .5800 & 5158 & .4378 & 3728 & 16.667 & .7047 & .6611 & .6353 & .7866 \\
\hline 1.028 & 5485 & .4969 & .4345 & 3692 & 2.410 & .5800 & .5158 & .4378 & 3728 & 17.123 & .6728 & .6458 & .6070 & .5631 \\
\hline 1.037 & 5485 & .4969 & .4345 & 3692 & 2.463 & 5800 & .5158 & .4378 & 3728 & 17.606 & :6482 & .6201 & .5787 & .5346 \\
\hline 1.047 & 5485 & .4969 & .4345 & 3692 & 2.519 & .5800 & .5158 & $1378^{\circ}$ & 3728 & 18.116 & .5776 & .5635 & .5344 & .5026 \\
\hline 1.057 & 5485 & .4969 & .4345 & .3692 & 2.577 & 5800 & .5158 & .4378 & 3728 & 18.657 & .5226 & 5125 & .4943 & .4685 \\
\hline 1.067 & 5485 & .4969 & .4345 & 3692 & 2.639 & .5800 & .5158 & .4378 & 3728 & 19.231 & .4668 & .4613 & .4541 & .4399 \\
\hline 1.078 & 5485 & .4969 & .4345 & .3692 & 2.703 & .5800 & .5158 & .4378 & 3728 & 19.841 & .4666 & .4217 & .4210 & .4140 \\
\hline 1.088 & 5485 & .4969 & .4345 & 3692 & 2.770 & .5800 & .5158 & .4378 & 3728 & 20.492 & .4666 & .4217 & .3992 & 3924 \\
\hline 1.099 & 5485 & .4969 & .4345 & 3692 & 2.841 & .5800 & .5158 & .4378 & 3728 & 21.186 & .4666 & .4217 & .3836 & 3766 \\
\hline 1.110 & 5485 & .4969 & .4345 & .3692 & 2.915 & .5800 & .5158 & .4378 & 3738 & 21.930 & -4666 & .4217 & .3739 & 3648 \\
\hline 1.121 & 5485 & .4969 & .0345 & 3692 & 2.994 & 5800 & .5158 & .4378 & 3738 & 22.727 & .4666 & .4217 & .3667 & 3532 \\
\hline 1.133 & .5647 & .5008 & .4345 & 3692 & 3.077 & .5800 & .5158 & .4378 & 3738 & 23.585 & .4666 & .4217 & .3667 & 3440 \\
\hline 1.144 & 5647 & .5008 & .4345 & 3692 & 3.165 & .5800 & .5158 & .4378 & 3738 & 24.510 & .4666 & .4217 & .3667 & 3336 \\
\hline 1.156 & 5647 & .5008 & .4345 & 3692 & 3.257 & .5800 & .5158 & .4378 & 3738 & 25.510 & .4666 & -.4217 & .3667 & 3336 \\
\hline 1.168 & .5647 & .5008 &., 4345 & .3692 & 3.356 & .5800 & .5158 & .4378 & 3738 & 26.596 & .4666 & .4217 & .3667 & .3336 \\
\hline 1.181 & .5647 & 5008 & .4345 & .3692 & 3.460 & .5800 & .5158 & .4378 & 3738 & 27.778 & .4666 & .4217 & .3667 & .3336 \\
\hline 1.193 & .5647 & .5008 & .4345 & .3692 & 3.571 & .5800 & .5158 & .4378 & 3738 & 29.070 & .4666 & -4217 & .3667 & .3336 \\
\hline 1.206 & 5647 & .5008 & .4345 & 3692 & 3.690 & .5800 & .5158 & .4378 & .3738 & 30,488 & .4666 & .4217 & .3667 & .3336 \\
\hline 1.220 & .5647 & .5008 & .4345 & 3692 & 3.817 & .5800 & .5158 & .4378 & .3738 & 32.051 & .4666 & .4217 & 3667 & .3336 \\
\hline 1.233 & .5647 & .5008 & .4345 & .3692 & 3.953 & .5800 & .5158 & .4378 & 3738 & 33.784 & .4666 & .4217 & .3667 & .3336 \\
\hline 1.247 & .5647 & 5008 & .4345 & .3692 & 4,098 & .5800 & .5158 & .4378 & 3738 & 35.714 & .4666 & .4217 & .3667 & .3336 \\
\hline 1.261 & .5647 & 5008 & .4345 & .3692 & 4.255 & .5800 & .5158 & .4378 & 3738 & 37.879 & -4666 & .4217 & .3667 & .3336 \\
\hline 1.276 & .5647 & 5008 & .4345 & .3692 & 4,425 & .5800 & .5158 & 4378 & .3738 & 40.323 & .4666 & .4217 & .3667 & 3336 \\
\hline 1.290 & .5647 & .5008 & .4345 & .3692 & 4.608 & 5800 & .5158 & .4378 & 3738 & 43.103 & .3174 & 3151 & .3110 & 3336 \\
\hline L.30s & .5647 & 5008 & .4345 & .3692 & .4 .808 & .5800 & .5158 & .4378 & .3738 & 46.296 & .2926 & .2929 & .2929 & .2930 \\
\hline 1.321 & .5647 & 5008 & .4345 & .3692 & 5.025 & .5800 & .5158 & .4378 & 3738 & 50,000 & .2786 & .2794 & .2810 & .2829 \\
\hline 1.337 & .5647 & .5008 & .4345 & 3692 & 5.263 & .5800 & .5158 & .4378 & .3738 & & & & & \\
\hline 1.353 & .5647 & .5008 & .4345 & 3692 & 5.525 & .5800 & .5158 & .4378 & .3738 & & & & & \\
\hline 1.370 & 5647 & .5008 & .4345 & .3692 & 5.814 & .5800 & .5158 & .4378 & 3738 & & & & & \\
\hline 1.387 & .5647 & .5008 & .4345 & 3692 & 6.135 & .5800 & .5158 & .4378 & 3738 & & & & & \\
\hline 1.404 &.$\$ 647$ & .5008 & .4345 & .3692 & 6.494 & 5800 & .5158 & -4378 & .3738 & & & & & \\
\hline $1: 422$ & .5647 & .5008 & .4345 & 3692 & 6.397 & .5800 & .5158 & .4378 & .3738 & & & & & \\
\hline 1.441 & 5647 & .5008 & .4345 & 3692 & 7.353 & 5800 & .5158 & .4378 & .3738 & & & & & \\
\hline 1.460 & .5647 & .5008 & .4345 & 3692 & 7.874 & 6062 & 5356 & .4451 & .3738 & & & & & \\
\hline
\end{tabular}


FIGURE C-11

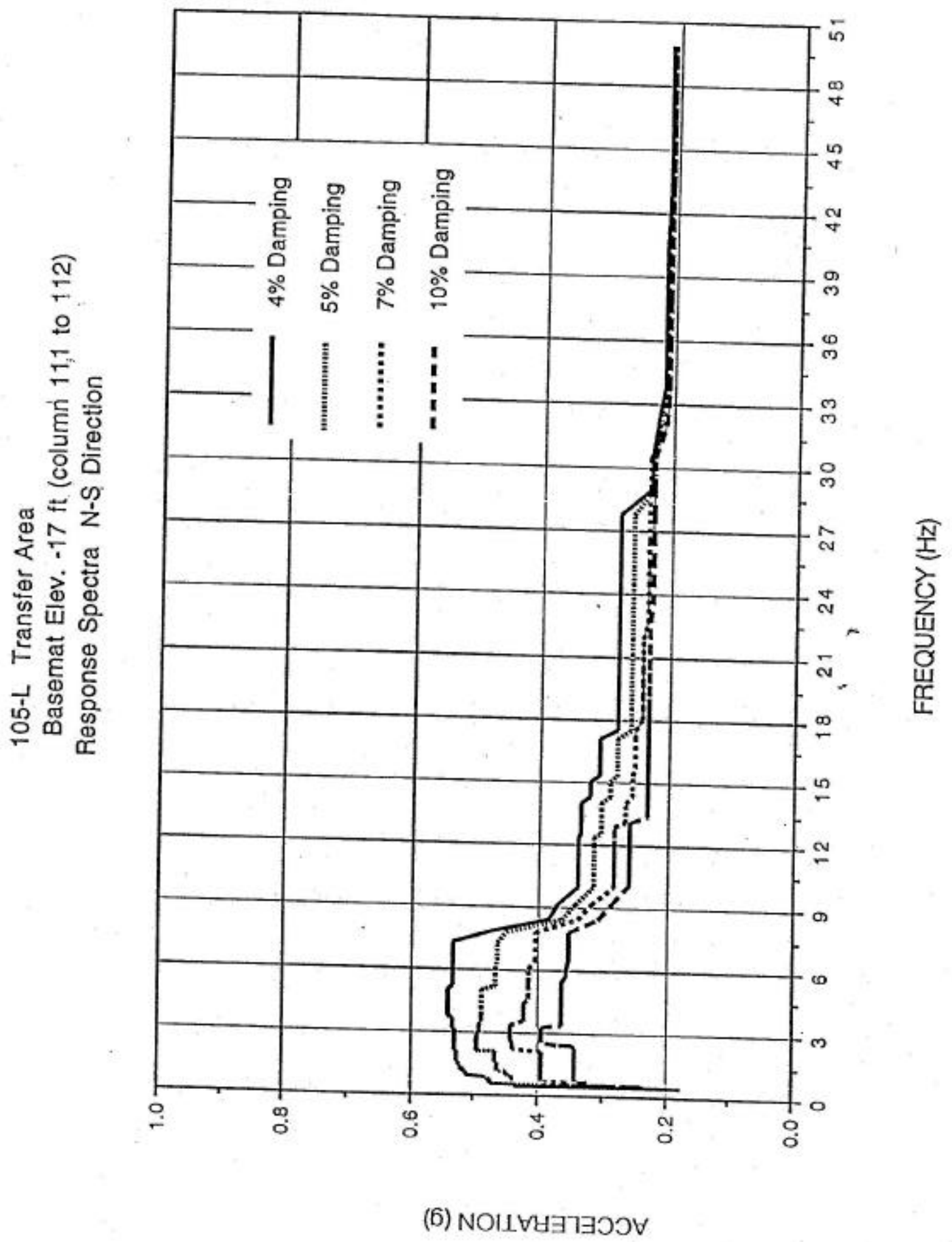


WSRC-TR-97-00289

Revision 0

Page C22 of C30

TABLE C-11

105-L. TRANSFER AREA (Column 111 to 112)

Elov. -17 it BAsemat RESPONSE SPECTAA

N-S DIRECTON

\begin{tabular}{|c|c|c|c|c|}
\hline $\begin{array}{l}\text { FEOUECY } \\
(\mathrm{Hz})\end{array}$ & 4\% DAMPNG & $\begin{array}{l}\text { CCELEFUTION } \\
5 \% \text { OAMPING }\end{array}$ & (p) & $10 \times$ DAMPING \\
\hline 0.500 & 0.220 & 0.218 & 0.198 & 0.175 \\
\hline 0.510 & 0.334 & 0.227 & 0.207 & 0.183 \\
\hline 0.521 & 0.334 & 0.235 & 0.214 & 0.188 \\
\hline 0.532 & 0.334 & 0.251 & 0.225 & 0.195 \\
\hline 0,543 & 0.334 & 0.268 & 0.240 & 0.208 \\
\hline 0.556 & 0,334 & 0.282 & .0 .254 & 0.220 \\
\hline $0.56 a$ & 0.435 & 0.204 & 0.265 & 0.231 \\
\hline 0.581 & 0.435 & 0.304 & 0.276 & 0.241 \\
\hline 0.595 & 0.435 & 0.313 & 0.265 & 0.250 \\
\hline 0.810 & 0.435 & 0.321 & 0.203 & 0.310 \\
\hline 0.625 & 0.474 & 0.435 & 0.301 & 0.319 \\
\hline 0.641 & 0.474 & 0.435 & 0.317 & 0.319 \\
\hline 0.658 & 0.474 & 0.435 & 0.338 & 0.319 \\
\hline 0.676 & 0.474 & 0.435 & 0.356 & 0.319 \\
\hline 0.604 & 0.474 & 0.435 & 0.368 & 0.319 \\
\hline 0.714 & 0.474 & 0.435 & 0.371 & 0.319 \\
\hline 0.735 & 0.474 & 0,441 & 0.394 & 0.340 \\
\hline 0.962 & 0.481 & 0.441 & 0,394 & 0.340 \\
\hline 1.000 & 0.514 & 0.443 & 0.394 & 0.340 \\
\hline 1.067 & 0.514 & 0.451 & 0.394 & 0.340 \\
\hline 1.233 & 0.514 & 0.451 & 0.394 & 0.340 \\
\hline 2.358 & 0.530 & 0.493 & 0.439 & 0.340 \\
\hline 2.410 & 0.530 & 0.493 & 0.430 & 0.340 \\
\hline 2.463 & 0.530 & 0.493 & 0.439 & 0.347 \\
\hline 2.519 & 0.530 & 0,493 & 0.439 & 0.368 \\
\hline 2.577 & 0.530 & 0.493 & 0.439 & 0.395 \\
\hline 3.460 & 0.532 & 0.492 & 0.443 & 0.362 \\
\hline 3.571 & 0.532 & 0.486 & 0.421 & 0.362 \\
\hline 5.025 & 0.539 & 0.486 & 0.415 & 0.362 \\
\hline 5.263 & 0.533 & 0.467 & 0.415 & 0.362 \\
\hline 5.525 & 0.533 & 0.467 & 0.415 & 0.362 \\
\hline 5.614 & 0.533 & 0.467 & 0.415 . & 0.357 \\
\hline 6.135 & 0.533 & 0.467 & 0.415 & 0.357 \\
\hline 6.494 & 0.533 & 0.462 & 0.405 & 0.353 \\
\hline 6,897 & 0.533 & 0.462 & 0.405 & 0.353 \\
\hline 7.353 & 0,533 & 0.462 & 0.405 & 0.353 \\
\hline 7.874 & 0,478 & 0.451 & 0.405 & 0.353 \\
\hline 8,475 & 0.385 & 0.363 & -0.338 & 0.308 \\
\hline 9.174 & 0.371 & 0.341 & 0.318 & 0.287 \\
\hline 10.000 & 0.338 & 0,313 & 0.282 & 0.261 \\
\hline 10.163 & 0.338 & 0.313 & 0.282 & 0.257 \\
\hline 10.331 & 0.338 & 0,313 & 0.282 & 0.257 \\
\hline 10.504 & 0.330 & 0.313 & 0.282 & 0.257 \\
\hline 10.684 & 0.338 & 0.313 & 0.202 & 0.257 \\
\hline 10.870 & 0.336 & 0.313 & 0.282 & 0.257 \\
\hline 11.062 & 0.338 & 0.313 & 0.282 & 0.257 \\
\hline 11.261 & 0.338 & 0.313 & 0.282 & 0.257 \\
\hline 11.468 & 0.338 & 0.313 & 0.282 & 0.257 \\
\hline 11.682 & 0.338 & 0.313 & 0.282 & 0.257 \\
\hline 14.368 & 0.320 & 0.291 & 0.253 & 0.228 \\
\hline 14.706 & 0.320 & 0.291 & 0.253 & 0.220 \\
\hline 17.606 & 0.278 & 0.258 & 0.251 & 0.228 \\
\hline 18.116 & 0.278 & 0.258 & 0.240 & 0.228 \\
\hline 19.841 & 0.278 & 0.256 & 0.240 & 0.228 \\
\hline 20.492 & $0.27 \mathrm{~B}$ & 0.250 & 0.240 & 0,228 \\
\hline 21.186 & 0.278 & 0.258 & 0.240 & 0.228 \\
\hline 21.030 & 0.278 & $0.25 \mathrm{~B}$ & 0.240 & 0.228 \\
\hline 22.727 & 0.278 & 0.258 & 0.234 & 0.228 \\
\hline 23.585 & 0.278 & 0.250 & 0.234 & 0.226 \\
\hline 32,051 & 0.221 & 0.218 & 0,213 & 0.210 \\
\hline 33.704 & 0.213 & 0.210 & 0,207 & 0.206 \\
\hline 35.714 & 0.213 & 0.210 & 0.207 & 0.206 \\
\hline 37.879 & 0.213 & 0.210 & 0.207 & 0.206 \\
\hline 46.296 & 0.208 & 0.207 & 0.207 & 0.206 \\
\hline 50,000 & 0.206 & 0.207 & 0.207 & 0.207 \\
\hline
\end{tabular}


FIGURE C-12

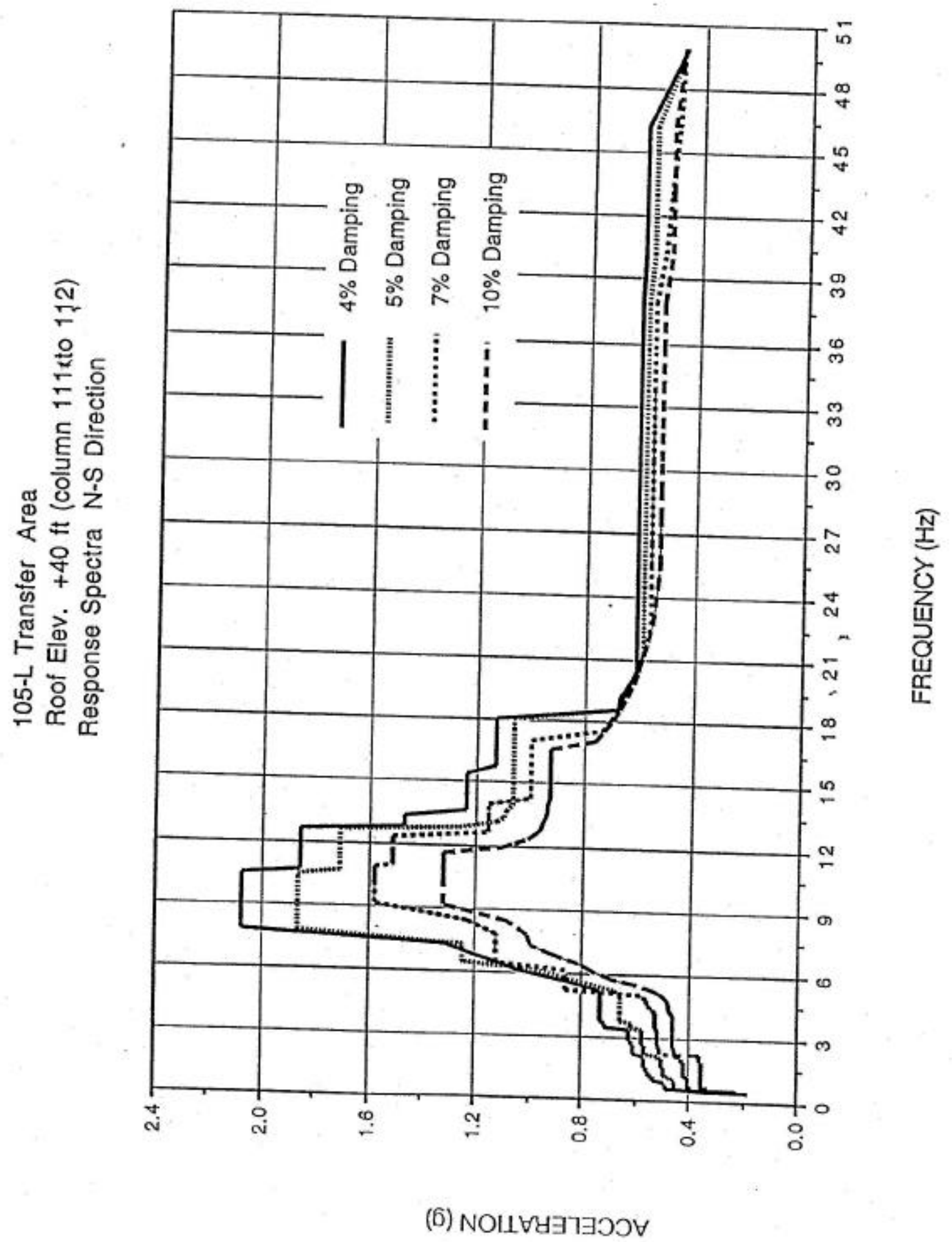


WSRC-TR-97-00289

Revision 0

Page C24 of C30

TABLE C-12

105-L. TRANSFEA AREA (Column 111 to 112)

Elev. t40 th foot RESPONSE SPECTRA

N-S DIRECTION

\begin{tabular}{|c|c|c|c|c|}
\hline $\begin{array}{l}\text { PUBXUACY } \\
(\mathrm{Hz})\end{array}$ & 4\% DAMPNG & $\begin{array}{l}\text { CCELERATION } \\
\text { 5\% DAMPNG }\end{array}$ & $\begin{array}{l}\text { (o) } \\
\text { T* DAMPING }\end{array}$ & $10 \%$ OAMPNG \\
\hline 0.500 & 0.231 & 0.220 & 0.201 & 0.178 \\
\hline 0,510 & 0.340 & 0.230 & 0.210 & 0.184 \\
\hline 0.521 & 0.340 & 0.238 & 0.216 & 0.191 \\
\hline 0.532 & 0.340 & 0.254 & 0.228 & 0.197 \\
\hline 0.543 & 0,340 & 0.271 & 0.243 & 0.209 \\
\hline 0.556 & 0.340 & 0.285 & 0.256 & 0.221 \\
\hline 0.568 & 0,439 & 0.296 & 0.267 & 0.233 \\
\hline 0.581 & 0,439 & 0.310 & 0.278 & 0.243 \\
\hline 0,505 & 0,430 & 0.317 & 0.287 & 0.251 \\
\hline 0.610 & 0.439 & 0.324 & 0.205 & 0.324 \\
\hline 0.625 & 0,481 & 0.432 & 0.305 & 0.324 \\
\hline 0.641 & 0.481 & 0.439 & 0,321 & 0.324 \\
\hline 0.658 & 0.481 & 0.430 & 0.306 & 0.324 \\
\hline 0.676 & 0.481 & 0.439 & 0.388 & 0.324 \\
\hline 0,694 & 0,481 & 0.439 & 0.388 & 0.324 \\
\hline 0.714 & 0.481 & 0.439 & 0.388 & 0.324 \\
\hline 0.735 & 0.481 & 0.450 & 0.403 & 0.340 \\
\hline 0.962 & 0.495 & 0.450 & 0.403 & 0.348 \\
\hline 1.000 & 0.530 & 0.457 & 0,403 & 0.349 \\
\hline 1.067 & 0.530 & 0.462 & 0.403 & 0.349 \\
\hline 1.233 & 0.531 & 0.472 & 0.403 & 0.349 \\
\hline 2.358 & 0.610 & 0.568 & 0.507 & 0.454 \\
\hline 2.410 & 0.610 & 0.568 & 0.507 & 0.454 \\
\hline 2.463 & 0.610 & 0,568 & 0.507 & 0.454 \\
\hline 2.519 & 0.610 & 0.568 & 0.507 & 0.454 \\
\hline 2.577 & 0.610 & 0.568 & 0.507 & 0.456 \\
\hline 3.460 & 0.710 & 0.614 & 0.527 & 0.456 \\
\hline 3,571 & 0.710 & 0,614 & 0,527 & 0.456 \\
\hline 5.025 & 0.720 & 0.653 & 0.550 & $0.49 \mathrm{~B}$ \\
\hline 5.263 & 0.736 & 0.680 & 0.863 & 0.536 \\
\hline 5.525 & 0.826 & 0.776 & 0.863 & 0.607 \\
\hline 5.814 & 0.803 & 0.856 & 0.863 & $0.690^{\circ}$ \\
\hline 6.135 & 1.045 & 0.967 & 0.863 & 0.763 \\
\hline 6.494 & 1.111 & 1.242 & 1.126 & 0.608 \\
\hline 6.097 & 1.219 & 1.242 & 1.126 & 0.909 \\
\hline 7.353 & 1.312 & 1.242 & 1.126 & 0.995 \\
\hline 7.874 & 2.073 & 1.858 & 1.126 & 1.009 \\
\hline 8.475 & 2.073 & 1.858 & 1.227 & 1.085 \\
\hline 0.174 & 2.073 & 1.658 & 1.576 & 1.316 \\
\hline 10.000 & 2.073 & 1.858 & 1.576 & 1.316 \\
\hline 10.163 & 2.073 & 1.858 & 1.576 & 1.316 \\
\hline 10.331 & 2.073 & 1.858 & 1.576 & 1.916 \\
\hline 10.504 & 2.073 & 1.858 & 1.576 & 1.316 \\
\hline 10.684 & 1.854 & 1.700 & 1.576 & 1.316 \\
\hline 10.870 & 1.854 & 1.700 & 1.576 & 1.316 \\
\hline 11.062 & 1.854 & 1.700 & 1.511 & 1.316 \\
\hline 11.261 & 1.854 & 1.700 & 1,511 & 1.316 \\
\hline 11,468 & 1.854 & 1.700 & 1.511 & 1.316 \\
\hline 11.682 & 1.854 & 1.700 & 1.511 & 1.316 \\
\hline 14.368 & 1,234 & 1.065 & 0.995 & 0,027 \\
\hline 14.706 & 1.234 & 1.065 & 0.996 & 0.927 \\
\hline 17.606 & 1.134 & 1.065 & 0.741 & 0.726 \\
\hline 16,116 & 1.134 & 1.065 & 0.707 & 0,696 \\
\hline 19.841 & 0.642 & 0.638 & 0.633 & 0.625 \\
\hline 20.402 & 0,615 & 0.612 & 0,609 & 0.604 \\
\hline 21.186 & 0.615 & 0.583 & 0.560 & 0.586 \\
\hline 21.930 & 0.615 & 0.593 & 0.571 & 0.571 \\
\hline 22.727 & 0.615 & 0.593 & 0.564 & 0,558 \\
\hline 23.505 & 0.615 & 0.593 & 0.564 & 0.548 \\
\hline 32.051 & 0.615 & 0.593 & 0,564 & 0.536 \\
\hline 33.784 & 0.615 & 0.503 & 0.564 & 0.536 \\
\hline 95.714 & 0.615 & 0.593 & 0.564 & 0.538 \\
\hline 37.879 & 0.615 & 0.593 & 0.564 & 0.536 \\
\hline 46.296 & 0.607 & 0.574 & 0.492 & 0,509 \\
\hline 50.000 & 0.467 & 0.469 & 0.471 & 0.474 \\
\hline
\end{tabular}


FIGURE C-13

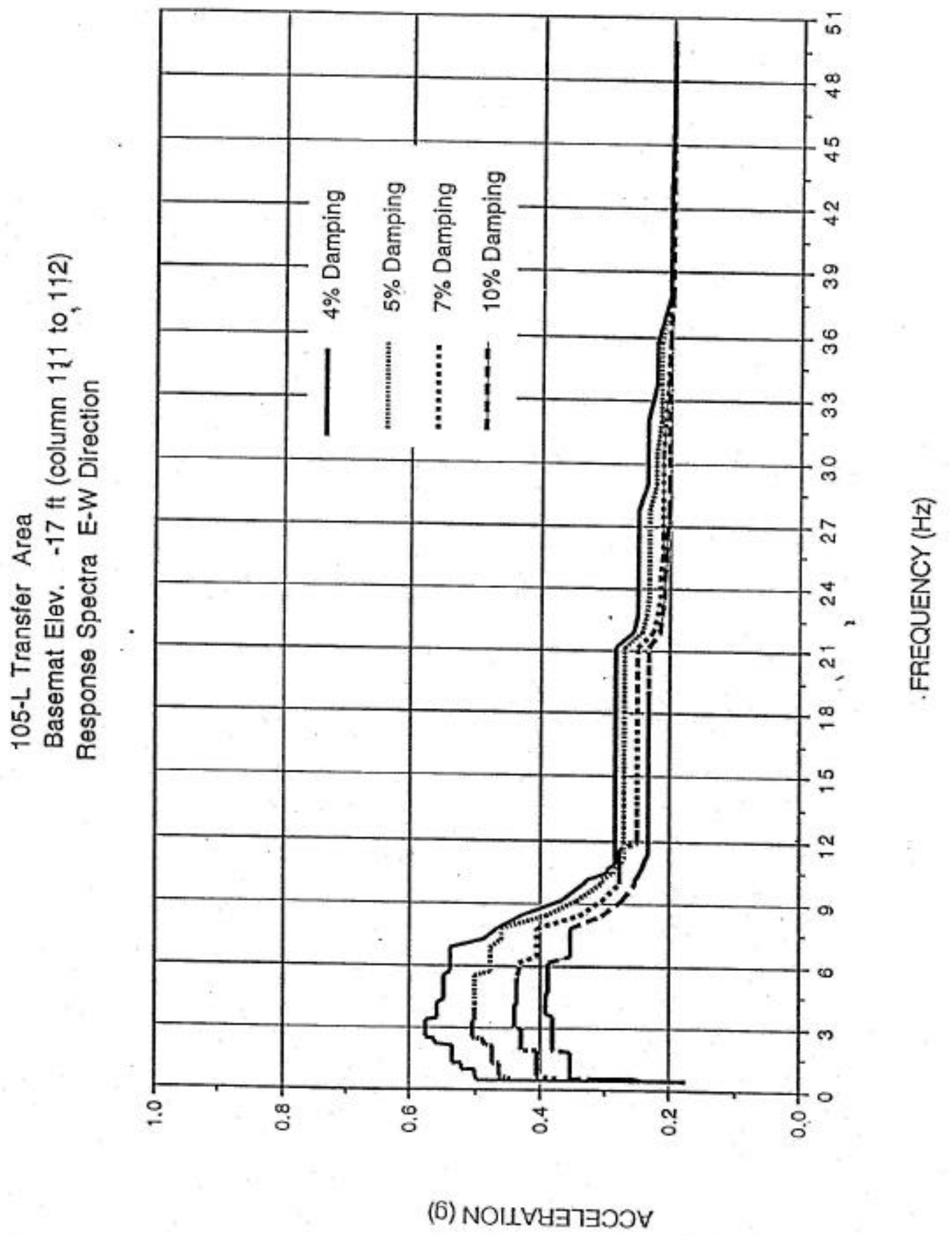


WSRC-TR-97-00289

Revision 0

Page C26 of C30

TABLE C-13

105-L TRANSFER AREA (Column 11110 112)

Elov, -17 th BAsemat RESPONSE SPECTRA E-W DIRECTION

\begin{tabular}{|c|c|c|c|c|}
\hline $\begin{array}{l}\text { FFEDECY } \\
(\mathrm{Hz})\end{array}$ & 4X. DAMPNAG & $\begin{array}{l}\text { CCELEPATION } \\
\text { S\% OAMPNA }\end{array}$ & 7\% DNMPNG & $10 \%$ DAMPINC \\
\hline 0.500 & 0.241 & 0.221 & 0.181 & 0.173 \\
\hline 0.510 & 0.274 & 0.250 & 0.214 & 0,180 \\
\hline 0.521 & 0.207 & 0.274 & 0.234 & 0.100 \\
\hline 0.532 & 0.322 & 0.206 & 0.371 & 0.204 \\
\hline 0.543 & 0.354 & 0.321 & 0.371 & 0.220 \\
\hline 0.556 & 0,484 & 0.444 & 0.371 & 0.259 \\
\hline 0.568 & 0.494 & 0.144 & 0.371 & 0.257 \\
\hline 0.581 & 0.494 & 0.444 & 0.371 & 0.273 \\
\hline 0.595 & 0.484 & 0.444 & 0.371 & 0.206 \\
\hline 0.610 & 0,494 & 0.414 & 0.371 & 0.296 \\
\hline 0.625 & 0.494 & 0.444 & 0.371 & 0.304 \\
\hline 0,641 & 0.494 & 0.444 & 0.371 & 0.307 \\
\hline 0.658 & 0.494 & 0.444 & 0.374 & 0.351 \\
\hline 0,676 & 0.498 & 0.461 & 0.404 & 0,351 \\
\hline 0.624 & 0.498 & 0.461 & 0.104 & 0.351 \\
\hline 0,714 & 0.498 & 0.461 & 0,404 & 0.351 \\
\hline 0.735 & 0.498 & 0.461 & 0.404 & 0.351 \\
\hline 0.962 & 0.496 & 0.461 & 0.404 & 0.351 \\
\hline 1.000 & 0.490 & 0,461 & 0.404 & 0,351 \\
\hline 1.067 & 0.518 & 0.461 & 0.404 & 0.351 \\
\hline 1.233 & 0,516 & 0.461 & 0,404 & 0,351 \\
\hline 2.350 & 0.559 & 0.484 & 0.427 & 0.380 \\
\hline 2.410 & 0.559 & 0.484 & 0.427 & 0.380 \\
\hline 2.463 & 0.550 & 0.484 & 0.427 & 0.380 \\
\hline 2.519 & 0,559 & 0.484 & 0.427 & 0.380 \\
\hline 2.577 & 0.574 & 0.501 & 0.427 & 0,390 \\
\hline 3.460 & $0,5 \mathrm{s8}$ & 0.400 & 0.437 & 0.380 \\
\hline 3.571 & 0.558 & 0.499 & 0.437 & 0,380 \\
\hline 5.025 & 0,545 & 0.499 & 0.433 & 0.385 \\
\hline 5.263 & 0.545 & 0.489 & 0.433 & 0.385 \\
\hline 5.525 & 0.545 & 0.499 & 0.433 & 0.385 \\
\hline 5.814 & 0.535 & 0.474 & 0.429 & 0.385 \\
\hline 6.135 & 0.535 & 0.474 & 0.429 & 0,385 \\
\hline 6.484 & 0.535 & 0.474 & 0.404 & 0.351 \\
\hline 6.897 & 0.535 & 0.474 & 0.404 & 0.351 \\
\hline 7.353 & 0.486 & 0,457 & 0,404 & 0.351 \\
\hline 7.874 & 0.463 & 0.457 & 0,404 & 0.351 \\
\hline 0.475 & 0.427 & 0.388 & 0.339 & 0.304 \\
\hline 9.174 & 0.264 & 0.340 & 0.304 & 0.272 \\
\hline 10.000 & 0.330 & 0.305 & 0.276 & 0.254 \\
\hline 10.163 & 0.326 & 0.304 & 0.276 & 0.253 \\
\hline 10.331 & 0.313 & 0.207 & 0.276 & 0.250 \\
\hline 10.504 & 0.298 & 0.288 & 0.276 & 0.246 \\
\hline 10.684 & 0.294 & 0.284 & 0.276 & 0.244 \\
\hline 10.870 & 0.285 & 0.277 & 0.276 & 0.211 \\
\hline 11.062 & 0.284 & 0.273 & 0.276 & 0.239 \\
\hline 11.261 & 0.284 & 0.270 & 0.276 & 0.236 \\
\hline 11.468 & 0.284 & 0.269 & 0.276 & 0.233 \\
\hline 11.682 & 0.284 & 0.269 & 0.276 & 0.231 \\
\hline 14,368 & 0.284 & 0.269 & 0.249 & 0.231 \\
\hline 14.706 & 0.284 & 0.269 & 0.249 & 0.231 \\
\hline 17.606 & 0.284 & 0.269 & 0.249 & 0.231 \\
\hline 18.116 & 0.284 & 0.269 & 0.219 & 0.231 \\
\hline 19.841 & 0.284 & 0.260 & 0.249 & 0.231 \\
\hline 20.402 & 0.284 & 0.269 & 0.249 & 0.231 \\
\hline 21.186 & 0.284 & 0.269 & 0,249 & 0.231 \\
\hline 21.930 & 0.256 & 0.243 & 0.224 & 0.214 \\
\hline 22.727 & 0.248 & 0.235 & 0.210 & 0.212 \\
\hline 23.585 & 0.248 & 0.232 & 0.217 & 0.209 \\
\hline 32,051 & 0.294 & 0.216 & 0.200 & 0.203 \\
\hline 33.784 & 0.222 & 0.216 & 0,203 & 0.203 \\
\hline 35.714 & 0.222 & 0.216 & 0.200 & 0.203 \\
\hline 37.079 & 0,202 & 0.201 & 0.200 & 0.199 \\
\hline 46.296 & 0.200 & 0.199 & 0.198 & 0.198 \\
\hline 50,000 & 0.200 & 0.199 & 0.197 & 0.197 \\
\hline
\end{tabular}


FIGURE C-14

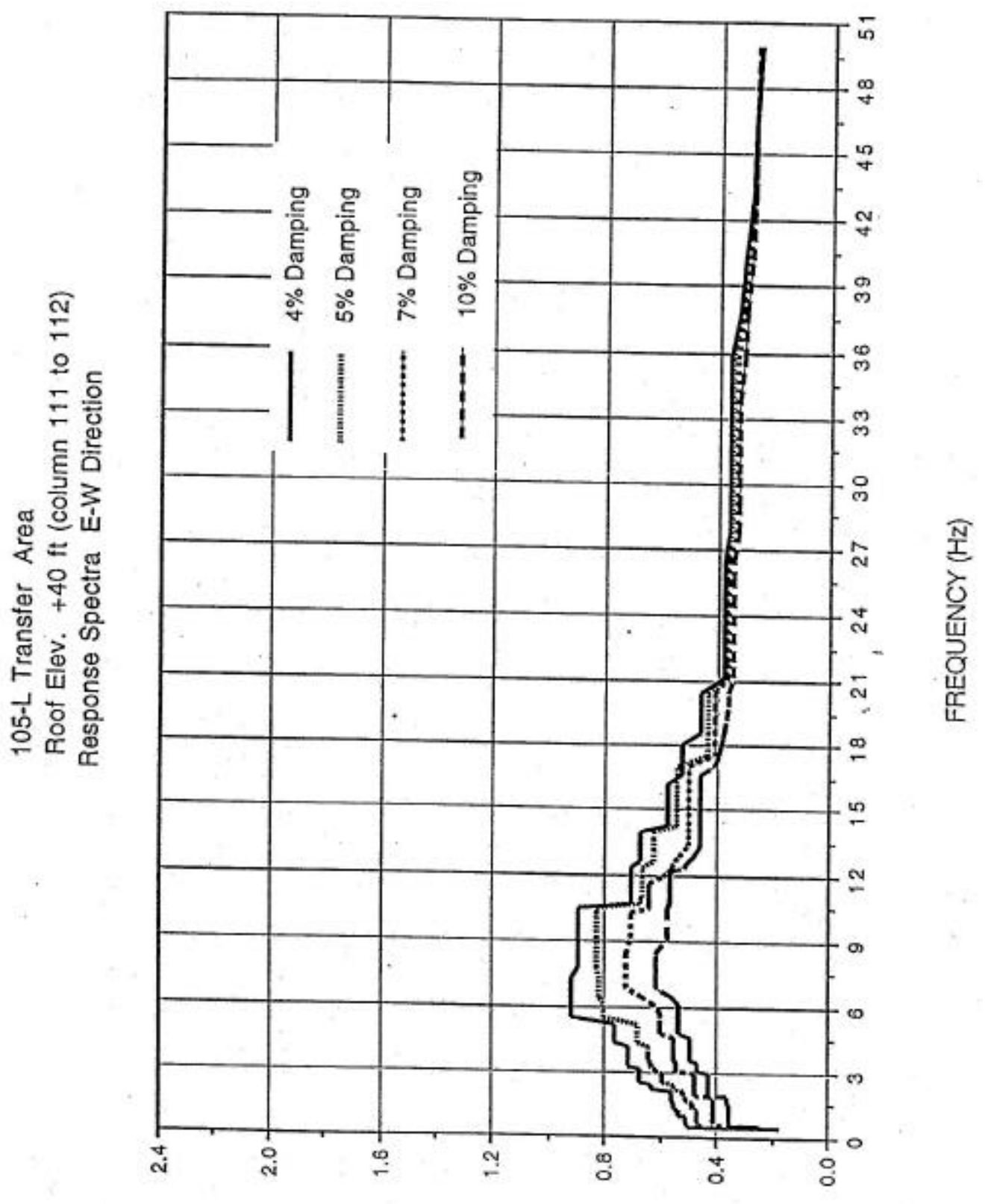

(6) NOLVYส7تOOV 
WSRC-TR-97-00289

Revision 0

Page C28 of C30

TABLE C-14

105-L. TRANSFER AREA (Column 111 to 112)

Elev, +40 ft Root RESPONSE SPECTRA

E-W DIRECTION

\begin{tabular}{|c|c|c|c|c|}
\hline $\begin{array}{c}\text { FEQDECY } \\
(\mathrm{Hz})\end{array}$ & 4XOANPNG & $\begin{array}{l}\text { CCELERATION } \\
\text { 5\% DAMPING }\end{array}$ & $\begin{array}{l}\text { (9) } \\
7 \times \text { DMMPNG }\end{array}$ & $10 x$ DNNPING \\
\hline 0,500 & 0.243 & 0.223 & 0.102 & 0.173 \\
\hline 0.510 & 0.274 & 0.251 & 0.215 & 0.181 \\
\hline 0.520 & 0.207 & 0.445 & 0.235 & 0.102 \\
\hline 0,532 & 0.323 & 0.445 & 0.373 & 0.205 \\
\hline 0.543 & 0.356 & 0.445 & 0.373 & 0.222 \\
\hline 0.556 & 0.497 & 0,447 & 0.373 & 0.240 \\
\hline 0,568 & 0.497 & 0.447 & 0.373 & 0.258 \\
\hline $0.5 \mathrm{Bi}_{1}$ & 0.497 & 0.447 & 0.373 & 0.273 \\
\hline 0.595 & 0.497 & 0.447 & 0.373 & 0.287 \\
\hline 0.610 & 0.407 & 0.447 & 0.373 & 0.298 \\
\hline 0.625 & 0.497 & 0.447 & 0.373 & 0.307 \\
\hline 0.641 & 0.497 & 0.447 & 0.373 & 0.310 \\
\hline 0.658 & 0.497 & 0.447 & 0.377 & 0.356 \\
\hline 0.676 & 0.506 & 0.466 & 0.410 & 0.356 \\
\hline 0.694 & 0.506 & 0.468 & 0.410 & 0.356 \\
\hline 0.714 & 0.506 & 0.468 & 0.410 & 0.356 \\
\hline 0.735 & 0.500 & 0.468 & 0.410 & 0.356 \\
\hline 0.862 & 0.506 & 0.468 & 0.410 & 0.356 \\
\hline 1.000 & 0.506 & 0.468 & 0.410 & 0.350 \\
\hline 1.067 & 0.530 & 0.460 & 0.410 & 0.356 \\
\hline 1.233 & 0.530 & 0.468 & 0.410 & 0.356 \\
\hline 2.358 & 0.632 & 0.548 & 0.476 & 0.427 \\
\hline 2.410 & 0.632 & 0.548 & 0.476 & 0.427 \\
\hline 2.463 & 0.632 & 0.548 & 0.476 & 0.427 \\
\hline 2.519 & 0.632 & 0.548 & 0.476 & 0.427 \\
\hline 2.577 & 0.673 & 0.587 & 0.476 & 0,427 \\
\hline 3.460 & 0.712 & 0.634 & 0.540 & 0.407 \\
\hline 3.571 & 0.712 & 0,636 & 0.551 & 0.492 \\
\hline 5,025 & 0.761 & 0.676 & 0.600 & 0.534 \\
\hline 5.263 & 0.761 & 0.676 & 0.600 & 0.534 \\
\hline 5.525 & 0.919 & 0.800 & 0.600 & 0.534 \\
\hline 5.814 & 0.919 & 0.800 & $0,000^{\circ}$ & 0.534 \\
\hline 6.135 & 0.919 & 0.809 & 0.622 & 0.534 \\
\hline 6.494 & 0.010 & 0.820 & 0.666 & 0.555 \\
\hline 6.697 & $0.81 \theta$ & 0.820 & 0.721 & 0.611 \\
\hline 7.353 & 0.919 & 0.820 & 0.721 & 0.611 \\
\hline 7.074 & 0.891 & 0.825 & 0.721 & 0.611 \\
\hline 8.475 & 0.091 & 0.825 & 0.721 & 0.611 \\
\hline 0.174 & 0,891 & 0.825 & 0.704 & 0,570 \\
\hline 10.000 & 0.891 & 0.325 & 0.704 & 0.570 \\
\hline 10.163 & 0.891 & 0.825 & 0.704 & 0.570 \\
\hline 10.331 & 0.891 & 0.625 & 0.704 & 0.570 \\
\hline 10,504 & 0.891 & 0.825 & 0.635 & 0.570 \\
\hline 10.684 & 0,703 & 0.674 & 0.635 & 0.568 \\
\hline 10.870 & 0.703 & 0.668 & 0.635 & 0.568 \\
\hline 11.062 & 0.703 & 0.666 & 0.635 & $0.56 a$ \\
\hline 11.261 & 0.703 & 0.666 & 0.635 & 0.568 \\
\hline 11.468 & 0.703 & 0.666 & 0.635 & 0.568 \\
\hline 11.682 & 0.703 & 0.666 & 0.635 & 0.560 \\
\hline 14.368 & 0.573 & 0.540 & 0.498 & 0.461 \\
\hline 14.706 & 0.573 & 0.540 & 0.498 & 0.461 \\
\hline 17.606 & 0.526 & 0.438 & 0.408 & 0.397 \\
\hline 18.116 & 0.526 & 0.430 & 0.408 & 0.388 \\
\hline 19.041 & 0.457 & 0.430 & 0.408 & 0.364 \\
\hline 20.492 & 0.457 & 0,438 & 0.408 & 0.357 \\
\hline 21.186 & 0.378 & 0.369 & 0.358 & 0.348 \\
\hline 21.930 & 0.378 & $0.369^{\circ}$ & 0.357 & 0.345 \\
\hline 22.727 & $0.37 a$ & 0.360 & 0.357 & 0.345 \\
\hline 23.585 & 0.378 & 0.369 & 0.357 & 0.345 \\
\hline 32.051 & 0.364 & 0.353 & 0.339 & 0.327 \\
\hline 33.784 & 0.364 & 0.353 & 0.339 & 0.327 \\
\hline 35,714 & 0.364 & 0.353 & 0.339 & 0.310 \\
\hline 37.079 & 0.329 & 0.322 & 0.312 & 0.301 \\
\hline 46,296 & 0,276 & 0.277 & 0.276 & 0.275 \\
\hline 50.000 & 0.263 & 0.264 & 0.266 & 0.269 \\
\hline
\end{tabular}


FIGURE C-15

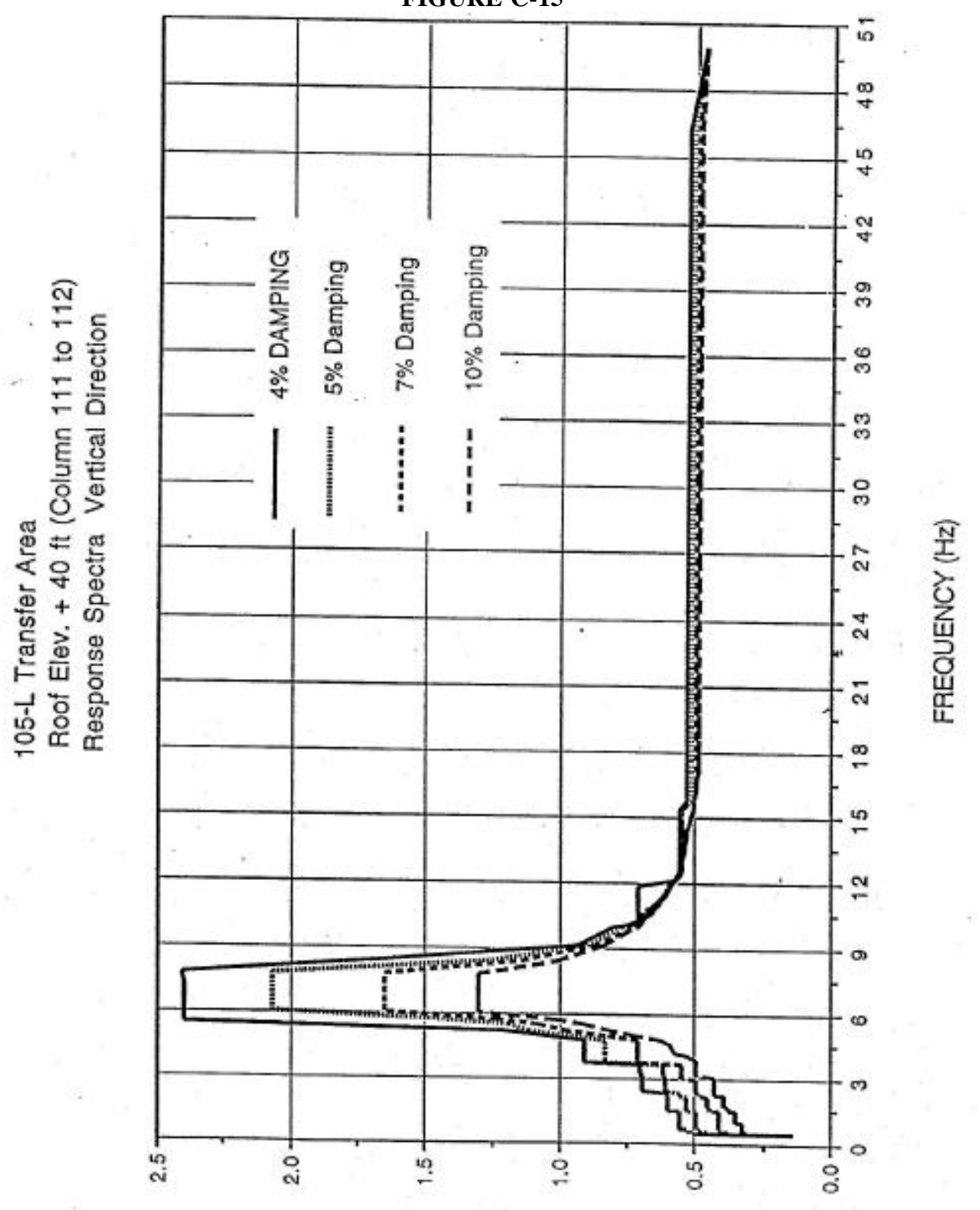

(6) NOIIV4ヨา 
WSRC-TR-97-00289

Revision 0

Page C30 of C30

TABLC C-15

IOS-L TRANSFER AREA (Colum 111 to 112) Elow, +40 At Roof RESPOWSE SPECTAA VERTICAL, DIRECTION

\begin{tabular}{|c|c|c|c|c|}
\hline \multirow{2}{*}{$\begin{array}{l}\text { Fresuer } \\
(\mathrm{Hz})\end{array}$} & \multicolumn{3}{|c|}{ ACCELEFUTKON (O) } & \multirow[b]{2}{*}{$10 \%$ DMMPSAG } \\
\hline & S\% OMMPZV: & 5*OAMPNG & 7x. OWMang & \\
\hline 0.500 & 0,190 & 0.160 & 0.160 & 0.140 \\
\hline 0.510 & 0.210 & 0.201 & 0.172 & 0,150 \\
\hline 0.521 & 0.503 & 0,453 & 0.378 & 0.161 \\
\hline 0.568 & 0,503 & 0.453 & 0.378 & 0.304 \\
\hline 0.581 & 0.503 & 0.453 & 0,378 & 0.304 \\
\hline 0.595 & 0.526 & 0,477 & 0.401 & 0.321 \\
\hline 0.610 & 0.526 & 0.477 & 0.401 & 0.321 \\
\hline 0.694 & 0.526 & 0.477 & 0,401 & 0.327 \\
\hline 0.750 & 0,554 & 0.406 & 0.400 & 0.327 \\
\hline 0.926 & 0.554 & 0,495 & 0.400 & 0.327 \\
\hline 0.962 & 0.554 & 0.406 & 0.409 & 0.327 \\
\hline 1.000 & 0.554 & 0.496 & 0.409 & 0.327 \\
\hline 1.078 & 0.554 & 0.496 & 0.412 & 0.351 \\
\hline 1.048 & 0.554 & 0.406 & 0.412 & 0.351 \\
\hline 1.133 & 0.554 & 0.406 & 0.412 & 0.351 \\
\hline 3.144 & 0,554 & 0.496 & 0.412 & 0.351 \\
\hline 1.158 & 0.554 & 0.496 & 0.412 & 0,351 \\
\hline 2.168 & 0,554 & 0.458 & 0.412 & 0.351 \\
\hline 1.520 & 0.554 & 0.496 & 0,412 & 0,351 \\
\hline 1.541 & 0.594 & 0.520 & 0.412 & 0,251 \\
\hline 1.946 & 0,594 & 0.52 .9 & 0.454 & 0.391 \\
\hline $1,9 \mathrm{a0}$ & 0.594 & 0.520 & 0.454 & 0,321 \\
\hline 2.262 & 0,800 & 0.555 & 0.489 & 0.391 \\
\hline 2.309 & 0.600 & 0.555 & 0.458 & 0,425 \\
\hline 2.356 & 0.600 & 0.555 & 0.489 & 0.425 \\
\hline 2.410 & 0,600 & 0,555 & 0.489 & 0.425 \\
\hline 2.483 & 0.690 & 0.604 & 0.491 & 0,425 \\
\hline 2.519 & 0,690 & 0,504 & 0.491 & 0.425 \\
\hline 3.077 & 0.680 & 0,604 & 0.545 & 0.427 \\
\hline 3.165 & 0.600 & 0,617 & 0,545 & 0.404 \\
\hline 3.571 & 0.704 & 0.617 & 0.545 & 0.494 \\
\hline 3.690 & 0.704 & 0,817 & 0.545 & 0.494 \\
\hline 3.817 & 0.902 & 0.029 & $0.709 \cdot$ & 0,494 \\
\hline 3.953 & 0,202 & 0,829 & 0.709 & 0.494 \\
\hline 4.425 & 0.902 & 0,829 & 0.709 & 0.501 \\
\hline 4,608 & 0.002 & 0.620 & 0.709 & 0.590 \\
\hline 4.808 & 0.902 & 0.829 & 0.710 & 0.610 \\
\hline 5.814 & 2.402 & 1,446 & 1.228 & 1,008 \\
\hline 6.135 & 2,402 & 2.067 & 1.646 & 1.280 \\
\hline 6.494 & 2.402 & 2.067 & 1.646 & 1.280 \\
\hline 0.897 & 2.402 & 2,067 & 1,646 & 1.298 \\
\hline 7.353 & 2.402 & 2.067 & 1.646 & 1.286 \\
\hline 7.674 & 2.402 & 2.067 & 1,648 & $1.20 a$ \\
\hline 6.475 & 1.652 & 1.244 & 1.133 & 1.005 \\
\hline 9.174 & 0,931 & 0.900 & 0.867 & 0.632 \\
\hline 10,000 & 0.700 & 0.764 & 0.721 & 0,715 \\
\hline 10.331 & 0.708 & 0.700 & 0.606 & 0.607 \\
\hline 10.504 & 0.708 & 0.698 & 0.636 & 0.674 \\
\hline $10,6 \mathrm{~B} 4$ & 0.700 & 0,692 & 0,675 & 0.601 \\
\hline 10,870 & 0.708 & 0.677 & 0.661 & 0.648 \\
\hline 11.062 & 0.700 & 0,652 & 0.645 & 0.634 \\
\hline 11,281 & 0.708 & 0.620 & 0.625 & 0,621 \\
\hline 14.466 & 0.708 & 0,614 & 0.614 & 0,600 \\
\hline 11.682 & 0.708 & 0,606 & 0,601 & 0.507 \\
\hline 11,205 & 0.708 & 0.594 & 0.508 & 0.5 .85 \\
\hline 12.136 & 0.571 & 0.570 & 0.572 & 0.573 \\
\hline 12.376 & 0.559 & 0.551 & 0.558 & 0,563 \\
\hline 12.626 & 0.559 & 0,551 & 0.548 & 0.554 \\
\hline 13.736 & 0.559 & 0.551 & 0,543 & 0.537 \\
\hline 14.045 & 0.552 & 0.551 & 0.543 & 0.532 \\
\hline 14,368 & 0.559 & 0.551 & 0,543 & 0,526 \\
\hline 14.706 & 0.559 & 0,551 & 0.543 & 0.518 \\
\hline 33,704 & 0.520 & 0.514 & 0.497 & 0.463 \\
\hline 25.714 & 0.526 & 0,514 & 0.497 & 0.483 \\
\hline 37.870 & 0.528 & 0,514 & 0.497 & 0,483 \\
\hline 40.323 & 0.525 & 0.514 & 0,497 & 0.483 \\
\hline 43.103 & 0.526 & 0,514 & 0,497 & 0.483 \\
\hline 46.205 & 0.526 & 0.514 & 0.407 & 0,103 \\
\hline So.000 & 0,461 & 0.467 & 0.470 & 0.471 \\
\hline
\end{tabular}

